This is a postprint of the following peer-reviewed publication:

Anquetin J. 2012. Reassessment of the phylogenetic relationships of basal turtles (Testudinata). Journal of Systematic Palaeontology 10: 3-45.

\title{
Reassessment of the phylogenetic interrelationships of basal turtles (Testudinata)
}

\author{
Jérémy Anquetin 1,2,3,4*
}

1UMR CNRS 7207 MNHN UPMC, Muséum National d'Histoire Naturelle, CP38, 8 rue Buffon, 75231 Paris cedex 05, France ${ }^{2}$ Department of Palaeontology, The Natural History Museum, Cromwell Road, London SW7 5BD, United Kingdom ${ }^{3}$ Research Department of Cell and Developmental Biology, UCL, University College London, Gower Street, London WC1E 6BT, United Kingdom

4UMR-CNRS 6143, Université de Rouen, IRESE A, Place Émile Blondel, 76821 Mont Saint Aignan, France

*Corresponding author: jeremy.anquetin@jurassica.ch

\begin{abstract}
Recent discoveries from the Late Triassic and Middle Jurassic have significantly improved the fossil record of early turtles. These new forms offer a unique opportunity to test the interrelationships of basal turtles. Nineteen fossil species are added to the taxon sample of the most comprehensive morphological phylogenetic analysis of the turtle clade. Among these additional species are recently discovered forms (e.g., Odontochelys semitestacea, Eileanchelys waldmani, Condorchelys antiqua), taxa generally omitted from previous analyses (e.g., chengyuchelyids, Sichuanchelys chowi) and species included in a phylogenetic analysis for the first time (Naomichelys speciosa and Siamochelys peninsularis). The coding of several characters is reassessed in light of recent observations, but also in order to reduce unwarranted assumptions on character and character state homologies. Additional characters from previous analyses, as well as five new ones, are also included, resulting in a data matrix of 178 characters scored for 86 turtle species and 7 fossil outgroups.

The data set resolves the relationships of most newly included taxa, with the exception of Sichuanchelys chowi and 'Chengyuchelys' dashanpuensis. The phylogenetic placement of Heckerochelys romani, Condorchelys antiqua and Eileanchelys waldmani as stem turtles more derived than Kayentachelys aprix but more basal than Meiolania platyceps and Mongolochelys efremovi is corroborated. The relationships of chengyuchelyids remain unclear and more investigation is needed regarding these forms, yet interestingly they are unstable with respect to stem turtles. In contrast to previous analyses, Arundelemys dardeni is placed within pleurosternids. Siamochelys peninsularis falls within xinjiangchelyids. Perhaps the most salient conclusion of the present study is the placement of Naomichelys speciosa as a basal member of a clade uniting meiolaniids, Mongolochelys efremovi and Otwayemys cunicularius. This clade of rather large stem turtles was spread worldwide during the Mesozoic at least and persisted up until the Pleistocene with meiolaniids.
\end{abstract}




\section{Introduction}

Turtles represent one of the most fascinating groups of amniotes. Their peculiar body plan, which at first glance may appear as a burden restricting the evolutionary possibilities of the group, has actually endured since the Late Triassic and allowed them to adapt successfully to various ecological niches (e.g., terrestrial, freshwater, bottom-walkers, costal, pelagic). In terms of evolution, turtles are particularly interesting because, for the moment, morphological, developmental and genetic studies are unable to reach consensus on the origin of the group within amniotes. Hence, without taking sides, turtles may be the last existing group of parareptiles (an once-large clade of anapsid reptiles including pareiasaurs and procolophonids), or they may represent a very derived group of eureptiles, possibly closely related to archosauromorphs, sauropterygians, or lepidosaurs (for a recent review see Rieppel 2008). For long, our knowledge of early turtles was limited mostly to material from the Late Triassic of Germany with Proganochelys quenstedti and Proterochersis robusta. The last two decades have witnessed the discovery of few additional early turtles such as Palaeochersis talampayansis (Late Triassic, Argentina), Australochelys africanus (Early Jurassic, South Africa), and Kayentachelys aprix (Early Jurassic, USA), which allowed to complete in some ways the turtle phylogenetic tree. However, it is the past few years which have been most exciting for specialists with the discovery of Late Triassic turtles more primitive than Proganochelys quenstedti (Li et al. 2008; Joyce et al. 2009), as well as of several species from the Middle Jurassic (Sukhanov 2006; Sterli 2008; Anquetin et al. 2009), a period from which the turtle fossil record is particularly sparse. These discoveries coincided with a major reappraisal of phylogenetic relationships within turtles by Joyce (2007). In this context, the present study proposes to reassess the phylogenetic interrelationships of early turtles by incorporating many additional species, including those discovered most recently, in a modified version of the matrix of Joyce (2007). These early fossil turtles represent the only direct evidence of the first stages of turtle evolution and understanding their interrelationships is of uppermost importance for all those interested in the place of turtles within amniotes.

The systematics of turtles has been significantly revised following the advent of cladistic methodology (Gaffney 1984). According to the most commonly accepted pattern of relationships (e.g., Gaffney 1975a, 1990, 1996; Gaffney \& Meylan 1988; Gaffney et al. 1991; Brinkman \& Wu 1999; Hirayama et al. 2000; Gaffney et al. 2007; Sterli et al. 2007), the phylogenetic stem of Testudines (sensu Joyce et al. 2004) is restricted to
Proganochelys quenstedti (Late Triassic, Germany), Palaeochersis talampayensis (Late Triassic, Argentina) and Australochelys africanus (Early Jurassic, South Africa). The recently described Late Triassic Odontochelys semitestacea should be added to the previous list as the most basal stem turtle ( $\mathrm{Li}$ et al. 2008). All remaining turtles are interpreted as members of the crown-group and classified as either pleurodires or cryptodires. The dichotomy between pleurodires and cryptodires was originally based on two main character complexes: the jaw closure mechanism (for a discussion see Joyce 2007) and the bracing of the braincase by palatoquadrate elements. In pleurodires, the trochlea of the main adductor muscle of the lower jaw is on the pterygoid and the braincase is braced by the quadrate, whereas in cryptodires the trochlea is on the quadrate and the braincase is braced by the pterygoid (Gaffney 1975a; Gaffney \& Meylan 1988). Within this context, Proterochersis robusta (Late Triassic, Germany) and Kayentachelys aprix (Early Jurassic, USA) are considered to be the most basal pleurodire and cryptodire, respectively (Gaffney 1975a; Gaffney et al. 1987).

However, some workers have also recovered a different pattern of relationships for basal turtles. The cladistic analysis of Dryden (1988) resulted in a phylogeny that departed significantly from those of Gaffney \& Meylan (1988) and Gaffney et al. (1991). According to Dryden (1988), Kayentachelys aprix, Meiolania platyceps, Pleurosternidae, Baenidae and Plesiochelyidae are all more basal than the pleurodire-cryptodire dichotomy. However, with the exception of Gauthier et al. (1989), Gaffney et al. (1991) and Joyce (2007), this work went mostly unnoticed as it was never formally published. Rougier et al. (1995) proposed that Proterochersis robusta was a stem turtle rather than a basal pleurodire.

Joyce (2007) was the first to propose a broad-scale phylogenetic analysis of the turtle clade in which all terminal taxa were species rather than broader taxonomic categories. This analysis was constructed to provide a comprehensive data set that summarised previous attempts to resolve turtle relationships. Most available phylogenetic analyses were scanned for characters and additional characters were derived from original descriptions of several species. This analysis included considerably more fossil taxa (i.e., 45 species) and more characters (i.e., 136) than previous studies. For all these reasons, the analysis of Joyce (2007) represented a major advance in our understanding of turtle relationships. The results partly confirmed the studies of Dryden (1988) and Rougier et al. (1995) in supporting an extensive stem for the turtle crown-group. According to Joyce (2007), Proterochersis robusta, Kayentachelys aprix, Meiolania platyceps, Mongolochelys efremovi and 
Meiolania platyceps, Mongolochelys efremovi and Kallokibotion bajazidi are stem turtles.

Joyce (2007) excluded from his analysis several early turtles whose relationships were uncertain: e.g., Indochelys spatulata (Datta et al. 2000), chengyuchelyids (e.g., Ye 1994; Danilov \& Parham 2008), Siamochelys peninsularis (Tong et al. 2002), and Otwayemys cunicularius (Gaffney et al. 1998). Additionally, several new basal turtles have been described since Joyce (2007) was published: Heckerochelys romani (Sukhanov 2006), Condorchelys antiqua (Sterli 2008; Sterli \& de la Fuente 2010), Odontochelys semitestacea ( $\mathrm{Li}$ et al. 2008) and Eileanchelys waldmani (Anquetin et al. 2009; Anquetin in press). Here, a new cladistic analysis is presented in order to test the relationships of these recently described and previously omitted species. The main objective of this analysis is to provide a more thorough exploration of basal turtle interrelationships. It is based largely on a revised version of Joyce's (2007) data matrix. The taxon sample has been increased to include all sufficiently wellknown pre-Late Jurassic species as well as some more recent species that might conceivably represent relatively basal taxa (see below). Seven non-chelonian taxa are included as outgroups in lieu of Proganochelys quenstedti and the hypothetical ancestor used by Joyce (2007). In order to encompass the newly added taxa, characters from previous analyses, as well as five new characters, are included in the data matrix. Finally, the coding of some characters used in Joyce (2007) is revised in an attempt to maximise the testing of homology statements by congruence.

The present study follows the anatomical terminologies of Zangerl (1969) and Gaffney (1972a, 1979), and the phylogenetic nomenclature of Joyce et al. (2004).

\section{Institutional abbreviations}

AMNH = American Museum of Natural History, New York, USA

BP = Bernard Price Institute for Palaeontological Research, University of the Witwatersrand, Johannesburg, South Africa

BSPG = Bayerische Staatssammlung für Paläontologie und Geologie, Munich, Germany

FMNH = Field Museum of Natural History, Chicago, USA

IVPP = Institute of Vertebrate Paleontology and Paleoanthropology, Beijing, China

MB = Museum für Naturkunde, Humboldt Universität, Berlin, Germany
MCZ = Museum of Comparative Zoology, Harvard University, Cambridge, Massachusetts, USA

MNA = Museum of Northern Arizona, Flagstaff, Arizona, USA

NHM = Natural History Museum, London, UK

NMS = National Museums of Scotland, Edinburgh, UK

PIN = Paleontological Institute of the Russian Academy of Sciences, Moscow, Russia

PMU = Museum of Evolution, Palaeontology section, Uppsala University, Uppsala, Sweden

SMNS = Staatliches Museum für Naturkunde, Stuttgart, Germany

TMM = Texas Memorial Museum, Austin, Texas, USA

UCPM = University of California Museum of Paleontology, Berkeley, California, USA

YPM = Yale Peabody Museum, Yale University, New Haven, Connecticut, USA

ZDM = Zigong Dinosaur Museum, Zigong, China

Taxa, characters, and coding strategy

\section{Taxon sampling}

The phylogenetic analysis of Joyce (2007) represents the first attempt to produce a broad-scale analysis of the relationships between some of the best-known Mesozoic turtles and representatives of extant turtle clades. Although Gaffney \& Meylan (1988) and especially Gaffney et al. (1991) attempted to reach a similar goal, Joyce's (2007) analysis was more comprehensive and less constrained by assumptions regarding the monophyly of numerous turtle clades because it included only species as terminal taxa. However, partly because it coincided with new discoveries and partly because of the aim of the study itself (i.e., producing a reasonably resolved, broadscale phylogeny), the taxon sampling of Joyce (2007) was incomplete, especially with respect to the most basal species whose potential relationships are poorly known.

The present analysis is intended to complement the analysis of Joyce (2007) by focusing on early turtles from the Triassic and Jurassic. In addition to the 45 fossil and 22 extant species scored by Joyce (2007), 19 other fossil species have been added to the present study. Four of these newly added species are stem turtles that have been discovered subsequent to the publication of Joyce (2007) and were therefore not included in that analysis: Odontochelys semitestacea (Li et al. 2008), Eileanchelys waldmani (Anquetin et al. 2009; Anquetin in press), Heckerochelys romani (Sukhanov 2006), and Condorchelys 
antiqua (Sterli 2008; Sterli \& de la Fuente 2010). The present analysis also scores Indochelys spatulata, a shellbased species from the Early Jurassic of India that is reputed to be close to Kayentachelys aprix (Datta et al. 2000). Asia (mostly China) has proven to be a prolific place for Middle Jurassic turtle discoveries. However, most of these species have been overlooked by phylogeneticists because they are often only briefly described, consist mostly of shells lacking associated crania, and are often difficult to access for direct observation. However, I was privileged to gain access to some specimens of several Chinese species (see Supplementary Material). The following species from Asia are included in the present phylogenetic analysis: Sichuanchelys chowi (Ye \& Pi 1997), Chengyuchelys baenoides (Young \& Chow 1953; Ye 1994), Chengyuchelys zigongensis (Ye 1982, 1994), 'Chengyuchelys' dashanpuensis (Fang 1987), Siamochelys peninsularis (Tong et al. 2002), Annemys levensis (Sukhanov 2000; Sukhanov \& Narmandakh 2006), Xinjiangchelys tianshanensis (Nessov 1995), and Xinjiangchelys qiguensis (Matzke et al. 2004). All but two of these species are from the Middle Jurassic, whereas $A$. levensis and $X$. qiguensis are from the Late Jurassic. In contrast to Matzke et al. (2004), Annemys levensis is included in the analysis rather than Annemys latiens (the type species of the genus; Sukhanov 2000; Sukhanov \& Narmandakh 2006) because cranial material of this species is known. Sichuanchelys chowi, Chengyuchelys zigongensis, 'Chengyuchelys' dashanpuensis and Siamochelys peninsularis are included in a phylogenetic analysis for the first time.

The remaining six newly added species are younger than the Jurassic. Arundelemys dardeni, from the Early Cretaceous of Maryland, USA, was described by Lipka et al. (2006) as a basal paracryptodire. The meiolaniids Ninjemys oweni from the Pleistocene of Australia and Niolamia argentina from the ?Eocene of Argentina (Gaffney 1996) are included in order to partly bridge the temporal and morphological gap between Meiolania platyceps and other turtles. Otwayemys cunicularius from the Early Cretaceous of Australia and Chubutemys copelloi from the Early Cretaceous of Argentina are included in order to test their proposed relationships with meiolaniids (Gaffney et al. 2007). Finally, Naomichelys speciosa, a large turtle from the Early Cretaceous of North America, is tentatively included in a phylogenetic analysis for the first time based on the observation of an undescribed, complete individual (FMNH PR273). This specimen is currently under formal description (S. D. Chapman, pers. comm. 2009), so I will not provide further details on the anatomy of this specimen here. Naomichelys speciosa is believed to be close to Helochelydra, a purported pancryptodire genus from the
Early Cretaceous of western Europe (e.g., Hirayama et al. 2000; Milner 2004).

Some of these newly added taxa are relatively poorly known and are represented only by shell material (e.g., Indochelys spatulata, Sichuanchelys chowi, Chengyuchelys baenoides, C. zigongensis, 'C.' dashanpuensis, Otwayemys cunicularius). This explains why they have been generally ignored in previous phylogenetic analyses, which have tended to concentrate on taxa with cranial remains. Their inclusion in the matrix results in an increased proportion of missing data (e.g., Otwayemys cunicularius and Sichuanchelys chowi can only be scored for $26 \%$ and $20 \%$ of characters, respectively), which can ultimately lead to an increased number of equally parsimonious trees and a decrease of resolution (e.g., Kitching et al. 1998). However, the aim of this analysis is not to produce a better-resolved phylogeny than previous studies, but to obtain a more comprehensive view of the relationships among the most basal members of the turtle clade. Sterli (2008) also proposed a modified version of Joyce's (2007) data set and scored three of the 19 species added herein (Heckerochelys romani, Condorchelys antiqua and Indochelys spatulata). Results from this study are discussed where appropriate.

\section{Outgroups}

Most phylogenetic analyses that considered the whole turtle clade have used Proganochelys quenstedti and/or a chimeric hypothetical ancestor consisting of a mixture between $P$. quenstedti and other amniotes as the outgroup (e.g., Dryden 1988; Gaffney et al. 1991; Rougier et al. 1995; Gaffney 1996; Shaffer et al. 1997; Brinkman \& Wu 1999; Hirayama et al. 2000; Gaffney et al. 2007; Joyce 2007; Sterli et al. 2007). Character polarisation aside, the general premise for outgroup comparison rests on the testing of ingroup monophyly, as long as more than one outgroup is used. However, this is not as relevant for turtles as it can be for other clades as turtle monophyly has not been seriously questioned (e.g., Gaffney \& Meylan 1988; Joyce 2007). Consequently, previous authors justified their choice of Proganochelys quenstedti as the outgroup because it was indisputably the most primitive turtle known to date, which in phylogenetic terms can be translated as 'P. quenstedti is the sister group to all other turtles' (e.g., Gaffney 1990; Joyce 2007). However, choosing Proganochelys quenstedti as the outgroup precludes any possibility that at least part of its morphology may be derived. In other words, choosing $P$. quenstedti as the outgroup equals accepting that this species represents the ancestral morphotype of turtles, because characters would be polarised based on the assumed plesiomorphic morphology of this species. 
With the recent discovery of Odontochelys semitestacea (Li et al. 2008), Proganochelys quenstedti can no longer be used as an outgroup for the rest of the turtle clade. The presence of teeth on the premaxilla, maxilla and dentary of $O$. semitestacea is strong evidence that it is more basal than $P$. quenstedti. Moreover, the postcranial morphology of $O$. semitestacea suggests that $P$. quenstedti may not represent the ancestral morphotype of turtles ( $\mathrm{Li}$ et al. 2008; but see also Reisz \& Head 2008), which contradicts some previous assumptions regarding the ancestral state of certain characters. For example, Joyce (2007: 50) proposed that a phalangeal formula with most digits reduced to two phalanges (a probable terrestrial adaptation) was plesiomorphic for turtles because it is the morphology found in Proganochelys quenstedti and Palaeochersis talampayensis. However, the manual phalangeal formula of Odontochelys semitestacea is 2-3-4-4-3, a condition found in no other turtle and one that is intermediate between the phalangeal formula found in most turtles (2-3-3-3-3) and the common reptilian formula of 2-3-4-5-3 (Li et al. 2008; see also character 175, below).

In order to avoid the a priori assumptions on character polarisation that would result from the choice of a basal turtle as the outgroup to all other species, outgroups must be taken from other amniote clades that are closely related to turtles. However, the highly derived body plan of turtles renders the analysis of their relationships with other amniotes difficult (for a review, see Rieppel 2008). Over the past thirty years, based on morphological arguments only, turtles have been proposed as close relatives of captorhinids (Gaffney \& McKenna 1979; Gaffney \& Meylan 1988; Gauthier et al. 1988), procolophonids (Reisz \& Laurin 1991; Laurin \& Reisz 1995), pareiasaurs (Lee 1993, 1995, 1996, 1997), sauropterygians (Rieppel \& deBraga 1996; deBraga \& Rieppel 1997; Rieppel \& Reisz 1999) and lepidosaurs (Müller, J. 2004; Hill 2005). In addition, numerous molecular studies have supported archosaurian affinities for turtles (for a complete list, see Rieppel 2008: 348). Sterli (2008) recently proposed a partial revision of the phylogenetic analysis of Joyce (2007) in which she used four non-chelonian outgroups (Sphenodon punctatus, Simosaurus gaillardoti, Anthodon serrarius and Owenetta kitchingorum). The present analysis follows this proposal and extends it to include representatives of all clades that have been proposed as the closest relatives of turtles. Anthodon serrarius (Lee 1997), Owenetta kitchingorum (Reisz \& Scott 2002) and Simosaurus gaillardoti (Rieppel 1994) represent pareiasaurs, procolophonids and sauropterygians, respectively. The extant Sphenodon punctatus is replaced by the Early Jurassic Gephyrosaurus bridensis (Evans 1980, 1981), a basal rhynchocephalian, as representative of lepidosaurs. A combined Captorhinidae is scored based on Captorhinus aguti (Fox \& Bowman 1966), Captorhinus laticeps (Heaton 1979) and Concordia cunninghami (Müller \& Reisz 2005). Araeoscelidia, representing basal 'diapsids', is scored based on the description of Araeoscelis by Reisz et al. (1984). Finally, early archosauromorphs are represented by Protorosaurus speneri (Gottmann-Quesada \& Sander 2009).

\section{Coding strategy}

Coding strategies have been intensively debated in the literature (e.g., Pimentel \& Riggins 1987; Meier 1994; Pleijel 1995; Wilkinson 1995c; Hawkins et al. 1997). Two main schools of thought on character construction can be identified (Wilkinson 1995c; Kitching et al. 1998): the first considers that characters should be coded as multistate, additive variables, whereas the second sees the characters only as binary variables. Those that defend multistate coding consider the characters as transformation series where each stage of the transformation is coded as a separate character state. Consequently, multistate characters are often ordered to respect this logical transformation series. The coding of multistate character requires the acceptance prior to the analysis that this transformation series is a true reflection of what happened during evolutionary history. Once locked into a unique multistate character, the hypothesised homology between character states can no longer be tested by congruence (Pleijel 1995). Apart from imposing the aforementioned constraints on the analysis, multistate coding also carries the risk of underweighting characters if two or more independent features are falsely united as a single multistate character (Wilkinson 1995c). Additionally, the question of the ordering of these multistate characters needs to be considered, because it imposes another level of constraint on the result of the analysis. Those that defend binary coding suggest that each variable should be coded as a separate character and tested for congruence against other variables (Pleijel 1995). The advantages of this methodology are that it minimises a priori assumptions about homology and that the character hierarchy emerges from the result rather than it being part of the original input (Kitching et al. 1998). However, this coding could potentially overweight some features, if characters are not independent from one another, and produce pseudo-parsimonious reconstructions (Meier 1994; Pleijel 1995; Wilkinson 1995c). Using one character construction or the other depends mostly on a philosophical choice and "neither approach has a monopoly of advantages or dangers" (Wilkinson 1995c: 307). Regardless of the preferred coding strategy, characters must be 
constructed with great care in order to minimise the effects of the identified pitfalls associated with each method (see above). Ideally, any character (binary or multistate) should code for a single feature in order to avoid supporting clades with assemblage of potentially unrelated variables and character states should be mutually exclusive, so that neither can co-occur in the same taxon (e.g., Farris et al. 1970; Pimentel \& Riggins 1987; Freudenstein 2005).

Although multistate coding imposes constraints on the resulting topologies, these constraints can be tolerated if the transformation series is sufficiently supported by the available data. However, the constraints imposed on the analysis by multistate characters (especially if they are ordered) could have a great influence on the result by restricting the possible topologies (usually toward a pattern that is congruent with our ideas about the interrelationships of the group under study). And even when characters are unordered, multistate coding makes homology assumptions that cannot be tested by congruence (e.g., Kitching et al. 1998; see above). In order to make as few assumptions as possible and to reduce these to basic, testable primary homology assessments, it has been chosen to code only binary characters in the present analysis. Hence, multistate characters from previous authors have been recoded herein as binary variables. Additionally, the present analysis follows the coding ' $C$ ' of Pleijel (1995), or 'conventional' coding of Hawkins et al. (1997), for which the presence/absence of a feature is coded separately from its various attributes (e.g., colour, shape). According to Hawkins et al. (1997), this coding represents the most appropriate method to translate morphological observations into cladistic characters.

\section{Characters}

Most characters used in the present study are taken or derived from Joyce (2007) and Sterli (2008), and modifications are discussed where appropriate. Eight characters from both Joyce (2007) and Sterli (2008) have been omitted from the present analysis. A specific rationale for omission is provided for each of these characters in the Supplementary Material. In order to encompass newly added taxa and outgroups, several characters have been taken from other studies (e.g., Matzke et al. 2004; Gaffney et al. 2007; Sterli et al. 2007) and details are also provided where appropriate. Additionally, five new characters are proposed.

Character 1. Nasals: 0 = present; 1 = absent (Joyce 2007, ch. 1).

Character 2. Medial contact of nasals: $0=$ nasals contact one another medially along their entire length; 1
$=$ medial contact of nasals partly or fully prevented by an anterior process of the frontal (Joyce 2007, ch. 2). Dorsetochelys delairi is scored state 1 , in contrast to Joyce (2007, state 0 ). The anterior process of the frontal in that species is similar in development to that of Arundelemys dardeni (Evans \& Kemp 1976: fig. 1A; Lipka et al. 2006: fig. 2A, F).

Character 3. Size of nasals: $0=$ dorsal exposure of nasals large, about the same size or larger than that of frontal; 1 = dorsal exposure of nasals greatly reduced relative to that of frontals (Joyce, 2007, ch. 3). According to personal observations (MNA V1558, MNA V2664 and TMM 43670-2) and recently published studies (Sterli \& Joyce 2007; Sterli 2008), Kayentachelys aprix has been scored state 1 in the present analysis, in contrast to Joyce (2007; state 0).

Character 4. Nasomaxillary sinus: $0=$ absent; $1=$ present (Gaffney 1996, ch. M12; Gaffney et al. 2007, ch. 4). Nasomaxillary sinuses are paired lateral pockets of the fossa nasalis extending posterolaterally into the bone and opening anteriorly just behind the apertura narium interna (Gaffney 1983: 403). They are formed by the nasal dorsally and maxilla ventrally. These structures are found only in meiolaniids: they are present in Meiolania platyceps and Ninjemys oweni, but it is not known whether or not they were present in Niolamia argentina (Gaffney 1996).

Character 5. Medial contact of prefrontals on the dorsal skull surface: $0=$ absent; $1=$ present, absence of contact between the nasal or apertura narium externa and the frontal (Joyce 2007, ch. 4).

Character 6. Prefrontal-vomer contact: $0=$ absent; $1=$ present (Joyce 2007, ch. 5). According to Gaffney et al. (1987) and Gaffney \& Jenkins (2010), a prefrontal-vomer contact is present in Kayentachelys aprix. Sterli \& Joyce (2007: 680) tentatively proposed that a prefrontal-vomer contact was present in Kayentachelys aprix. However, as stated by Sterli \& Joyce (2007) and confirmed by personal observation, the actual contact is not preserved in any specimen and the state of preservation of the material prevents any definitive conclusion on this matter. Consequently, Kayentachelys aprix is scored unknown in the present analysis.

Character 7. Prefrontal-palatine contact: 0 = present; 1 = absent (Laurin \& Reisz 1995, ch. 6; deBraga \& Rieppel 1997, ch. 20; Müller, J. 2004, ch. 130; Hill 2005, ch. 70; Joyce 2007, ch. 6).

Character 8. Foramen orbito-nasale: $0=$ absent; $1=$ present (Laurin \& Reisz 1995, ch. 10; Hill 2005, ch. 79). This character is included for outgroup comparison. The foramen orbito-nasale is an opening between the fossa 
nasalis and the fossa orbitalis that is usually formed by the prefrontal, palatine and maxilla in turtles. It may be entirely surrounded by bone, as it is usual in turtles (Gaffney 1979), or only developed as a notch, as in captorhinids (Laurin \& Reisz 1995). Most parareptiles have a foramen orbito-nasale that is developed between the prefrontal, lacrimal and palatine, whereas diapsids lack this foramen (Laurin \& Reisz 1995). The lacrimal also enters the margin of the foramen in Proganchelys quenstedti (Gaffney 1990).

Character 9. Dorsal prefrontal exposure: 0 = present; 1 = absent or near absent (modified from Joyce 2007, ch. 7). Joyce (2007) coded the dorsal prefrontal exposure as an ordered multistate character (dorsal prefrontal exposure large; reduced to small lappets; absent or near absent). Although it may seem logical at first glance to follow this coding (especially when considering the reduced prefrontal lappets of pleurosternids and Neurankylus eximius and the absence of dorsal exposure of the prefrontal in baenids), it almost automatically implies that the condition in baenids is derived from some group with state 1 , because deriving the baenids from a group with state 0 would cost one more step (in this case, $0 \rightarrow 1$-> 2). Running this multistate character unordered would still mean that homology assumptions between character states are made a priori and cannot be tested by congruence (see above). Hence, the development of the dorsal exposure of the prefrontal is coded with two binary characters (characters 9 and 10). That way only, congruence could say, as a result of the analysis, if the condition in baenids derives from a condition with small lappets or full dorsal exposure of the prefrontal (see Discussion). The present character codes for the presence or absence of dorsal exposure of the prefrontal. Only some baenids are known lack a dorsal exposure of the prefrontal.

Character 10. Dorsal prefrontal exposure: 0 = large; 1 $=$ reduced to small lappets (modified from Joyce 2007, ch. 7). See character 9 for a discussion. Most turtles have an exposure of the prefrontal on the dorsal skull roof and this exposure is usually large. However, the reduction of the dorsal prefrontal exposure to small lappets occurred several times within turtles. It is present in Kayentachelys aprix, Eileanchelys waldmani, Arundelemys dardeni, Dorsetochelys delairi, pleurosternids, Neurankylus eximius, Sinemys lens, Ordosemys leios, and at least some chelids. In contrast to Joyce (2007; state 0), Dorsetochelys delairi is scored state 1 in the present analysis. Taxa that lack a dorsal exposure of the prefrontal are scored inapplicable for this character.

Character 11. Lacrimal: 0 = present; 1 = absent (Joyce, 2007, ch. 9).
Character 12. Lacrimal: $0=$ elongate, enters the border of the external nares (apertura narium externa of turtles) and prevents the contact between maxilla and nasal and/or prefrontal; 1 = short, does not enter the border of the external nares and allows extensive contact between maxilla and nasal and/or prefrontal (Gauthier et al. 1988, ch. 33; Laurin \& Reisz 1995, ch. 9; deBraga \& Rieppel 1997, ch. 17; Lee 1997, ch. 26; Müller, J. 2004, ch. 6; Hill 2005, chs 98, 107). This character is included for outgroup comparison. With the exception of Proganochelys quenstedti, Palaeochersis talampayensis and Australochelys africanus, turtles lack a lacrimal bone and are consequently scored inapplicable for this character.

Character 13. Frontal contribution to orbit: $0=$ absent, contact between prefrontal and postorbital; 1 = present (Joyce 2007, ch. 10).

Character 14. Postfrontal: $0=$ present; $1=$ absent (Dryden 1988, ch. 26; Gaffney \& Meylan 1988, ch. A4-8; Gauthier et al. 1988, ch. 9; Hill 2005, ch. 77).

Character 15. Temporal fenestration: $0=$ absent; $1=$ present (Gauthier et al. 1988, chs 35, 37; Laurin \& Reisz 1995, chs 29, 30; deBraga \& Rieppel 1997, chs 50, 51; Lee 2001, chs 50, 51; Müller, J. 2004, chs 9, 17; Hill 2005, chs 109, 110; Sterli 2008, ch. 69). This character is included for outgroup comparison. Within the context of the present analysis, there is no reason to code two different characters (one for the upper, one for the lower fenestra) as is usual because both characters would have exactly the same repartition. This situation may change with a different choice of outgroup and in that case two characters should be coded. Temporal fenestration is present in Araeoscelidia (this analysis follows the scoring of Gauthier et al. 1988, Laurin \& Reisz 1995, deBraga \& Rieppel 1997, and Lee 2001, for the presence of temporal fenestrae in this group), Protorosaurus speneri, Simosaurus gaillardoti and Gephyrosaurus bridensis, and absent in Anthodon serrarius, Owenetta kitchingorum, Captorhinidae and all turtles.

Character 16. Parietal-squamosal contact: $0=$ present, upper temporal emargination absent or poorly developed; 1 = absent, upper temporal emargination well developed (Joyce 2007, ch. 11).

Character 17. Parietal contact with the pterygoid, epipterygoid, or palatine: $0=$ absent, foramen nervi trigemini usually developed as a notch; 1 = present, foramen nervi trigemini clearly formed by the processus inferior parietalis (Joyce 2007, ch. 12).

Character 18. Length of anterior extension of the lateral braincase wall: $0=$ short, processus inferior parietalis only produces a narrow strut anterior to the foramen nervi trigemini, usually absence of contact with 
palatine; 1 = elongate, processus inferior parietalis produces an extended process anterior to the foramen nervi trigemini, contact with palatine commonly present (Joyce 2007, ch. 13).

Character 19. Postparietal: $0=$ present; $1=$ absent (Dryden 1988, ch. 27; Gaffney \& Meylan 1988, ch. A4-3; Gauthier et al. 1988, ch. A36; Laurin \& Reisz 1995, ch. 4; deBraga \& Rieppel 1997, ch. 52; Müller, J. 2004, ch. 14; Hill 2005, ch. 93). This character is included for outgroup comparison. Postparietals are absent in all turtles, as well as in Protorosaurus speneri, Gephyrosaurus bridensis and Simosaurus gaillardoti.

Character 20. Jugal-squamosal contact: 0 = present; 1 = absent, contact between postorbital and quadratojugal commonly present (Joyce 2007, ch. 14).

Character 21. Jugal participation in the rim of the upper temporal emargination: 0 = absent; 1 = present, upper temporal emargination extensive (Joyce 2007, ch. 15).

Character 22. Deep lower temporal emargination extending above the upper limit of the cavum tympani, usually resulting in a loss of the quadratojugal: $0=$ absent; 1 = present (modified from Joyce, 2007, ch. 16). Character 16 of Joyce (2007) codes for the presence/ absence of a quadratojugal in relation to the development of a deep lower temporal emargination. According to his scoring, the quadratojugal is lost only in chelids and state 1 is a synapomorphy of this clade. However, as described by Joyce (2007: 14), the quadratojugal is also lost in some testudinoids as well as in Sinemys lens and S. gamera. This appears to be related to the lack of ossification of the 'temporal bar' in these taxa (Joyce 2007). In order to avoid confusion, the character has been reworded here.

Character 23. Quadratojugal-maxilla contact: $0=$ absent; 1 = present (Joyce 2007, ch. 17).

Character 24. Quadratojugal-squamosal contact below the cavum tympani: $0=$ absent; 1 = present (Gaffney \& Meylan 1988, ch. C11-3; Gaffney 1996, ch. M17; Joyce 2004, ch. Quadratojugal B; Sterli 2008, ch. 18). Meiolania platyceps and Ninjemys oweni are unique among turtles in having a posteroventral process of the quadratojugal that extends below the cavum tympani and contacts the squamosal. The condition in Niolamia argentina is unknown, although it is probable that this contact was present.

Character 25. Squamosal-supraoccipital contact: $0=$ absent; 1 = present (Joyce 2007, ch. 19).

Character 26. Meiolaniid 'horns': $0=$ absent; $1=$ present (Gaffney \& Meylan 1988, ch. C11-1; Gaffney 1996, ch. M5; Joyce 2004, ch. Squamosal B; Gaffney et al. 2007, ch. 16). The most striking feature of the meiolaniid skull is the development of horn-like projections of the squamosal (e.g., Gaffney 1983, 1996). In the present taxon sample, these structures are only found in Meiolania platyceps, Ninjemys oweni and Niolamia argentina.

Character 27. Postorbital-palatine contact: 0 = absent; 1 = present, foramen palatinum posterius situated posterior to the orbital wall (Joyce, 2007, ch. 20).

\section{A}

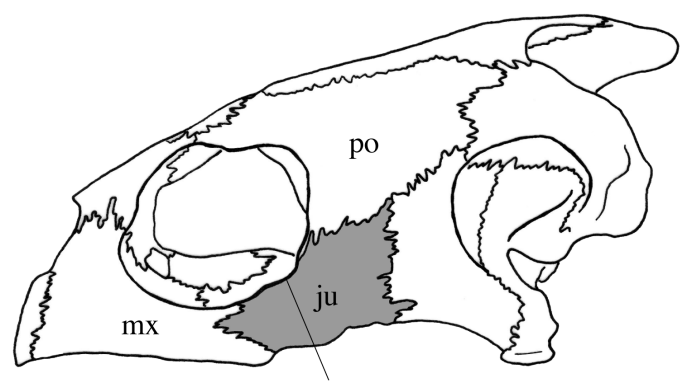

ch. $28(0)$

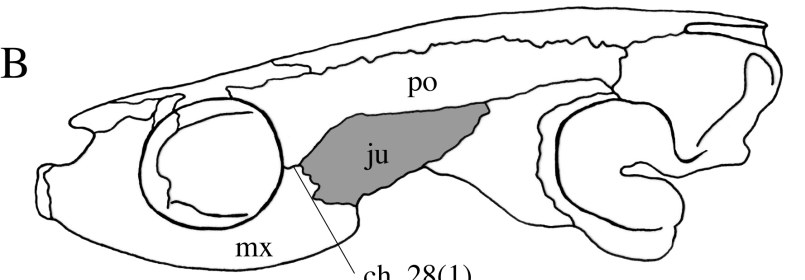

ch. 28(1)

Figure 1 - Illustration of states for character 28. Lateral views of the skull of Eretmochelys imbricata (A) and Pleurosternon bullockii (= Mesochelys durlstonensis Evans \& Kemp, 1975) (B). (A) redrawn from Gaffney (1979b) and (B) redrawn from Evans \& Kemp (1975) and Gaffney (1979b). Abbreviations: ju, jugal; mx, maxilla; po, postorbital.

Character 28 (Fig. 1). Postorbital-maxilla contact preventing the jugal from entering the orbital margin: $0=$ absent; 1= present (Gaffney \& Meylan 1988, ch. C6-4; Brinkman \& Nicholls 1991, ch. 13; Lyson \& Joyce 2009, ch. 18; see also Scheyer \& Anquetin 2008). A contact between the maxilla and postorbital that excludes the jugal from the orbital margin is relatively uncommon among turtles. It is known in at least three extant species: Platysternon megacephalum, Emys orbicularis and Malayemys subtrijuga (Gaffney 1979). Among fossil taxa scrutinised for this analysis, the occurrence of this contact is limited to some paracryptodires. A maxilla-postorbital contact is present in Arundelemys dardeni, Pleurosternon bullockii (= Mesochelys durlstonensis Evans \& Kemp, 1975), Glyptops plicatulus and the baenid Plesiobaena antiqua. This condition is also known in Eubaena cephalica (e.g., Gaffney 1972b; Lyson \& Joyce 2009). The morphology of Dinochelys whitei regarding this character is unclear 
(Brinkman et al. 2000: 272), whereas in Dorsetochelys delairi the jugal enters the orbital margin according to the reconstruction provided by Evans \& Kemp (1976).

Character 29. Postorbital-supratemporal contact: $0=$ present; 1 = absent (Gauthier et al. 1988, ch. 24; Laurin \& Reisz 1995, ch. 12; deBraga \& Rieppel 1997, ch. 30; Müller, J. 2004, ch. 184; Hill 2005, ch. 95). This character is included for outgroup comparison. Among the outgroups that have a supratemporal (see character 30), this contact is present in Anthodon serrarius and Owenetta kitchingorum, whereas it is absent in Captorhinidae and Araeoscelidia. Odontochelys semitestacea, Proganochelys quenstedti and Palaeochersis talampayensis are the only turtles where a supratemporal is known and this bone does not contact the postorbital in these species. Taxa that have lost the supratemporal are scored inapplicable for this character.

Character 30. Supratemporal: $0=$ present; $1=$ absent (Gaffney \& Meylan 1988, ch. A2-2; Gauthier et al. 1988, ch. 23; Laurin \& Reisz 1995, ch. 18; deBraga \& Rieppel 1997, chs 30, 53; Müller, J. 2004, ch. 21; Hill 2005, ch. 95; Joyce 2007, ch. 21).

Character 31. Subdivision of the apertura narium externa by an internarial process of the premaxilla only: 0 = present; 1 = absent (Joyce 2007, ch. 22). The present analysis differs from that of Joyce (2007; state 0 ) in scoring Meiolania platyceps and Kallokibotion bajazidi as lacking an internarial process formed by the premaxilla (state 1). Although the apertura narium externa is indeed divided in these two species, the separation appears to be formed by the nasal and premaxilla in $M$. platyceps (Gaffney 1983, 1996) and apparently by the nasal in $K$. bajazidi (Gaffney \& Meylan 1992). The condition in these two taxa is hypothesised as non-homologous to the plesiomorphic amniote condition (state 0 ) where the premaxillae send a dorsal internarial process that partly separates the nasals anteromedially (Gaffney 1996: 120). In the present analysis, the condition seen in meiolaniids is coded as a separate character (see character 32 ). In contrast to Joyce (2007; state 0), Sterli et al. (2007; state 0) and Sterli (2008; state 0), Australochelys africanus is scored unknown for this character. Personal observation of the holotype and only known specimen (BP/1/4933) reveals that it is not possible to determine which bones participate in the formation of the internarial septum in this species.

Character 32. Apertura narium externa subdivided by an internarial process formed by the nasal and premaxilla and somewhat recessed within the apertura: $0=$ absent; 1 = present (Gaffney 1996, ch. M15; modified from Sterli et al. 2007, ch. 1; Sterli 2008, ch. 23). An internarial process formed by the nasal and premaxilla is found only in Meiolania platyceps and Ninjemys oweni. The apertura narium externa is single in Niolamia argentina. The condition in Kallokibotion bajazidi appears nonhomologous to that of Meiolania platyceps: the internarial septum seems to be formed by the nasal only and it is not recessed within the apertura (Gaffney \& Meylan 1992; Gaffney 1996). In order to avoid favouring any scenario, $K$. bajazidi is scored unknown for this character. Naomichelys speciosa is also scored unknown for this character because, although as preserved the apertura is not subdivided in FMNH PR273, a small anteroventral process of the nasal is present and might represent the remnant of a medial septum dividing the apertura narium externa.

Character 33. Fusion of the premaxillae: $0=$ absent; 1 $=$ present (Joyce 2007, ch. 23).

Character 34. Foramen praepalatinum: 0 = present; 1 = absent (modified from Joyce 2007, ch. 24). Joyce (2007) coded an unordered multistate character for the presence/absence of the foramen praepalatinum (present; absent, premaxilla well ossified; absent, foramen intermaxillaris present). The foramen praepalatinum is present in the majority of turtles. In most cheloniids (here only in Chelonia mydas and Caretta caretta), the premaxillae are well ossified but the foramina praepalatinum are absent. A different morphology is found in Trionychia (sensu Joyce et al. 2004) in which the foramina praepalatinum appear to be absent because of the poorly ossified anterior palate forming a gaping single median opening called the foramen intermaxillaris (e.g., Gaffney 1979; Joyce 2007). Coding these three morphologies into a single multistate character assumes the homology between the three states (e.g., Pleijel 1995), so that somehow the foramen intermaxillaris corresponds to modified foramina praepalatinum. This homology statement is neither supported nor contradicted by available data. According to the coding strategy adopted here, this multistate character is recoded as two binary characters (characters 34 and 35). The present character codes for the presence/ absence of the foramen praepalatinum and representatives of Trionychia are scored inapplicable for it. Although Heaton (1979: fig. 7) identified a "prepalatal foramen" within the premaxilla of Captorhinus laticeps, the homology of the chelonian foramen praepalatinum in other reptiles has yet to be assessed and none of the recent phylogenetic analyses of amniote relationships consider this problem. Consequently, each outgroup is scored unknown for this character.

Character 35. Foramen intermaxillaris: $0=$ absent; $1=$ present (Meylan \& Gaffney 1989, ch. 8; modified from Joyce 2007, ch. 24). 
Character 36. Exclusion of the premaxillae from the apertura narium externa: 0 = absent; 1 = present (Joyce 2007, ch. 25). In contrast to Joyce (2007; state 0), Australochelys africanus is scored unknown for this character because this area of the skull is not preserved in the only known specimen (BP/1/4933).

Character 37. Distinct medial premaxillary hook along the labial margin of the premaxillae: $0=$ absent; $1=$ present (Joyce 2007, ch. 26).

Character 38. Accessory ridge on triturating surface of the maxilla: 0 = absent; 1 = present (Gaffney 1996, ch. M16; Hirayama et al. 2000, ch. 13; Gaffney et al. 2007, ch. 22). The triturating surface of the maxilla usually bears a lingual ridge that is variably developed among turtles (Gaffney 1979). Some meiolaniids have an additional ridge on the triturating surface (the accessory ridge of Gaffney 1983). Remarkably, Niolamia argentina lacks an accessory ridge (Woodward 1901; Gaffney 1996). Khosatzky (1997) proposed that Mongolochelys efremovi had an accessory ridge similar to that of meiolaniids. This proposition has been followed by subsequent workers (Hirayama et al. 2000; Gaffney et al. 2007; this work). Accessory ridges are also present sporadically within crown-group turtles in some pleurodires, testudinids, batagurids and emydids (Gaffney 1979, 1996).

Character 39. Teeth on the upper and lower jaws (maxilla, premaxilla and dentary): 0 = present; 1 = absent (Dryden 1988, ch. 3; Gaffney \& Meylan 1988, ch. A4-5; Gauthier et al. 1988, ch. A41; deBraga \& Rieppel 1997, ch. 3; Hill 2005, ch. 193; Sterli 2008, ch. 68). This character is primarily included for outgroup comparison. All turtles but Odontochelys semitestacea lack marginal teeth.

Character 40. Maxillary contribution to orbital margin: 0 = maxilla entirely or largely excluded from the orbital margin by a lacrimal-jugal contact; $1=$ maxilla forms most of the anteroventral edge of the orbit (Lee 1995, ch. 21; deBraga \& Rieppel 1997, ch. 15; Lee 1997, ch. 28; Müller, J. 2004, ch. 128; Hill 2005, ch. 103; Sterli et al. 2007, ch. 15).

Character 41. Vomer: $0=$ present; $1=$ absent or vestigial (modified from Joyce 2007, ch. 27). Joyce (2007) scored a single ordered multistate character for the morphology of the vomer (paired; single; single, greatly reduced or absent). This coding may appear logical in view of what is known of turtle phylogeny: a paired vomer is only found in most basal turtles, then all turtles have a single vomer and finally some groups lost the vomer. However, it implies a priori statements (i.e., ordering, as well as transformation assumptions that cannot be tested by congruence) that could be avoided. According to the methodology followed here, the presence/absence of the vomer (character 41) and the fact that this bone may be paired or single (character 42) are kept as separate characters. Within the analysed taxon sample, the vomer is missing or vestigial only in Pelomedusoides (i.e., Podocnemis expansa, Erymnochelys madagascariensis and Pelomedusa subrufa).

Character 42. Vomer: 0 = paired; 1 = single (modified from Joyce 2007, ch. 27). See character 41 for a discussion. Taxa that have lost the vomer are scored inapplicable for this character. According to Gaffney \& Kitching (1995) and personal observation (BP/1/4933), the vomer appears to have been paired in Australochelys africanus. However, this species is cautiously scored as unknown for this character until further evidence is available.
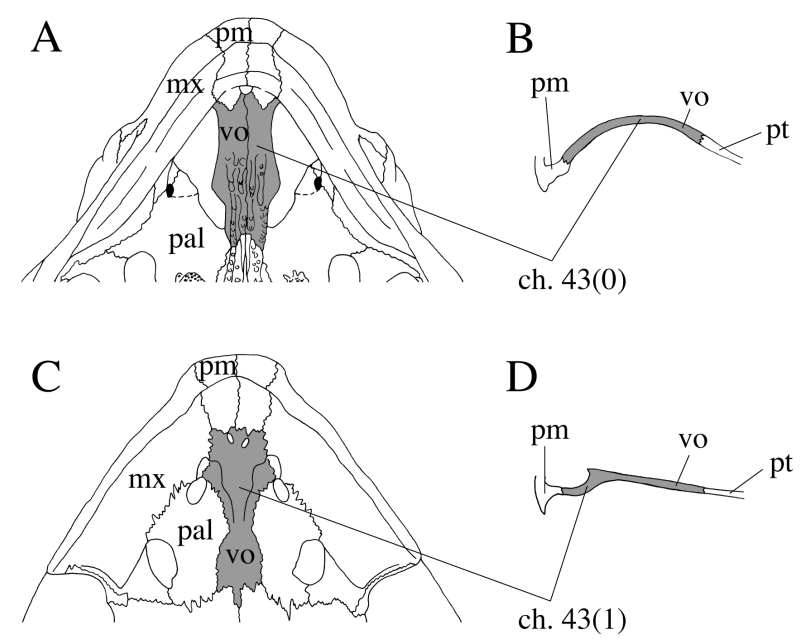

Figure 2 - Illustration of states for character 43. (A, B) Proganochelys quenstedti in ventral view (A) and sagittal section (B); (C, D) Chelydra serpentina in ventral view (C) and sagittal section (D). Modified from Anquetin et al. (2009). Abbreviations: $\mathrm{mx}$, maxilla; pal, palatine; pm, premaxilla; pt, pterygoid; vo, vomer.

Character 43 (Fig. 2). Vomer shape: 0 = flat blade, either convex dorsally or horizontal; $1=$ develops a ventral median septum and often a ventral horizontal plate contributing to an incipient secondary palate (new character). This character results directly from observation of the morphology in Eileanchelys waldmani (Anquetin et al. 2009; Anquetin in press). State 0 corresponds to the plesiomorphic amniote morphology and is found in basalmost turtles like Proganochelys quenstedti, Kayentachelys aprix and Eileanchelys waldmani. All more derived turtles, starting with Meiolania platyceps and Mongolochelys efremovi, have a vomer that acquires a three-dimensional shape by developing at least a ventral median septum dividing the meatus choanae and sometimes in addition a ventral horizontal plate (the 
dumbbell-shape of Gaffney 1979). Taxa that have lost the vomer are scored inapplicable for this character.

Character 44. Vomer-pterygoid contact in palatal view: 0 = present; 1 = absent, medial contact of palatines present (Joyce 2007, ch. 28). In contrast to Joyce (2007; states 0, 0, and 1, respectively), Australochelys africanus, Palaeochersis talampayensis and Sandownia harrisi are scored as unknown for this character. In Australochelys africanus, it is unclear whether the bone anterior to the interpterygoid vacuity is the pterygoid or the palatine (BP/ 1/4933). In Palaeochersis talampayensis, the vomerpterygoid contact is probably present, but it is not actually observable in the material (Sterli et al. 2007). In Sandownia harrisi, the development of an extensive secondary palate obscures the palate roof and prevents the proper scoring of this character (Meylan et al. 2000: figs 2 and 3). According to Gaffney \& Meylan (1992: 13), the vomer does not separate the palatines to meet the pterygoids in Kallokibotion bajazidi. This species is consequently scored state 1 in the present analysis, whereas it was scored state 0 in Joyce (2007). State 1 is also found in Chubutemys copelloi, Solnhofia parsonsi, 'Thalassemys' moseri, Santanachelys gaffneyi and Trionychia (sensu Joyce et al. 2004). Taxa that have lost the vomer are scored inapplicable for this character.

Character 45. Vomerine and palatine teeth: $0=$ present; 1 = absent (Joyce 2007, ch. 29).

Character 46. Palatine contribution to the anterior extension of the lateral braincase wall: 0 = absent; $1=$ present, well-developed (Joyce 2007, ch. 30).

Character 47. Ventromedial process of the quadrate and/or ventral expansion of the prootic that partly floors the cavum acustico-jugulare: $0=$ absent; 1 = present (modified from Joyce 2007, ch. 31). The cavum acusticojugulare corresponds to the medial part of the middle ear cavity in turtles. In basal forms, the cavum is not floored so that it is apparent in ventral view (e.g., Proganochelys quenstedti, Kayentachelys aprix, Eileanchelys waldmani). In more derived turtles, a flooring of the cavum acusticojugulare often develops and results in a more extensive bracing of the braincase posteriorly. However, two different morphologies exist within turtles (Gaffney 1979: 210). The flooring of the cavum acustico-jugulare can be produced by a more or less extensive posterior process of the pterygoid, the latter often contacting the basioccipital posteriorly. This morphology can be found in all pancryptodires as well as some basal turtles (e.g., Meiolania platyceps, Mongolochelys efremovi and Kallokibotion bajazidi). The second morphology consists of a generally less extensive flooring of the cavum acusticojugulare formed by a ventromedial process of the quadrate and/or a ventral expansion of the prootic. This morphology is found in most pleurodires. Gaffney (1975a) conceived this character complex in order to support the dichotomy between cryptodires and pleurodires, but subsequent workers recognised the absence of homology between these two morphologies and consequently refrained from coding them into a single multistate character (see Joyce 2007: 19). In contrast, Joyce (2007) coded a multistate character for the flooring of the cavum acustico-jugulare (absent; produced by pterygoid; produced by quadrate and prootic). His rationale is based on the fact that if the two derived morphologies are coded separately then the plesiomorphic state of each character is composite: for example in the case of the pleurodire-type flooring, the plesiomorphic state would encompasse both the absence of flooring found in basal turtles and the presence of a floor formed by the posterior process of the pterygoid (Joyce 2007: 19). Although the remark is accurate, this is actually not a problem. Because these two types of flooring are, according to available evidence, not homologous, the absent/present coding used here does not imply any assumption on the morphology of taxa scored absent, it simply asks whether or not a particular derived morphology is present in the considered taxa. The perspective of the present work is reversed relative to that of Joyce (2007): the coded feature is not whether the cavum is floored or not (i.e., the a posteriori interpreted character complex) but whether or not the quadrate produces a ventromedial process that braces the braincase (character 47), or whether or not the pterygoid has a well-developed posterior process that often contacts the basioccipital (character 60). The result of both derived states produces a similar morphology: the flooring of the cavum acustico-jugulare. In the present analysis, Notoemys laticentralis is scored state 0 because its basicranial morphology is very similar to that of Eileanchelys waldmani or Kayentachelys aprix, for example (Lapparent de Broin et al. 2007: fig. 2). This differs from Joyce (2007) who scored $N$. laticentralis as having the pleurodire-type flooring, which would correspond to state 1 in the present analysis.

Character 48. Central constriction of the middle ear by the quadrate: 0 = absent; 1 = present (Joyce 2007, ch. 32). Sterli (2008) proposed that characters 32 and 33 of Joyce (2007; characters 48 and 49 herein) were not independent and united them as her character 34. This may be true, but some morphological observations tend to indicate that it is possible to code two characters: in Australochelys africanus and Palaeochersis talampayensis, the cavum tympani is incipient but the central constriction of the quadrate around the columella auris has not begun (Gaffney \& Kitching 1995; Joyce 2007; Sterli et al. 2007). Until this matter is further investigated, the 
present analysis follows that of Joyce (2007) in coding two separate characters. In contrast to Joyce (2007), characters 48, 49, 50, 52, 53 and 54 (see below) are scored as unknown for Dinochelys whitei because the relevant morphological areas are not preserved on the only known skull of this species (Brinkman et al. 2000).

Character 49. Cavum tympani: $0=$ absent; 1 = present (modified from Joyce 2007, ch. 33). The development of the cavum tympani is very difficult to code because it appears to represent a truly continuous character. Proganochelys quenstedti is interpreted as lacking a cavum tympani (e.g., Gaffney 1990; Joyce 2007), although a shallow recessed area is present along the posterior edge of the quadrate (SMNS 16980). In Autralochelys africanus, an incipient cavum tympani is present as a well-defined recessed area along the posterior part of the quadrate (BP/1/4933). In Palaeochersis talampayensis, the cavum tympani is even more marked, although it does not reach the development seen in all other turtles (Sterli et al. 2007). Coding quantitative characters can be difficult when no real separation can be drawn between potential states, as it is the case for the development of the cavum tympani. Joyce (2007), following Rougier et al. (1995), coded an ordered multistate character with an intermediate state 'cavum tympani partly developed'. Joyce (2007) scored Australochelys africanus and Palaeochersis talampayensis as having the intermediate state, although the development of the cavum tympani is clearly not equivalent in both taxa (see above). In contrast, Rougier et al. (1995) assigned the intermediate state only to $P$. talampayensis, $A$. africanus being scored as lacking a cavum tympani. Given the fact that there is no consensus, only the absence/presence of the cavum tympani is scored here and both $A$. africanus and $P$. talampayensis are scored state 1.

Character 50. Precolumellar fossa: $0=$ absent; $1=$ present (Joyce 2007, ch. 34).

Character 51. Antrum postoticum: 0 = absent; $1=$ present (modified from Joyce 2007, ch. 35). Joyce (2007) coded an ordered multistate character for the development of the antrum postoticum and its constitution (absent; present, quadrate does not fully enclose the anterior perimeter of the antrum; present, quadrate fully encloses the anterior perimeter). According to Joyce's (2007) scoring, many pancryptodires are polymorphic (presenting states 1 and 2). Actually, Joyce scored taxa he was unable to observe firsthand as polymorphic (1/2) in order to express that they all have an antrum postoticum but that he was not sure of which bones formed its anterior perimeter (W. G. Joyce, pers. comm. 2010). Given that I did not myself have access to all relevant taxa, the construction of the anterior perimeter of the antrum postoticum is omitted herein and the present analysis follows the simple absent/ present coding of previous authors (Dryden 1988, ch. 29; Rougier et al. 1995, ch. 29). Sterli (2008, ch. 36) attempted to code the development of the antrum postoticum in a multistate character and scored it as incipient (her intermediate state) only in Kayentachelys aprix, rendering this state uninformative. Furthermore, no rationale was proposed for this coding.

Character 52. Incisura columellae auris: 0 = absent; 1 = present (modified from Joyce 2007, ch. 36). Joyce (2007) proposed an unordered multistate character for the absence/presence of the incisura columellae auris and its morphology (absent; present, open posteroventrally; present and closed, only enclosing the stapes; present and closed, enclosing both the stapes and the Eustachian tube). This is a systematic character that regroups all observed morphologies into one character complex, which can be used to recognise or emphasise differences between different groups. According to the coding strategy followed herein, such characters must be translated so as to minimise a priori assumptions on character evolution and possible linkage between characters that could constrain the resulting topology. The original multistate character of Joyce (2007) can be divided into three binary characters: the absence/ presence of the incisura (character 52); whether the incisura is open posteroventrally or closed (character 53); and, where the incisura is closed, whether the quadrate also encloses the Eustachian tube or not (character 54).

Character 53. Incisura columellae auris: $0=$ open posteroventrally; 1 = closed by the quadrate (modified from Joyce 2007, ch. 36). See character 52 for a discussion. Joyce (2007), Sterli (2008) and all previous authors who coded the morphology of the incisura columellae auris (see references in Joyce, 2007) followed Gaffney's (1983) description and scored Meiolania platyceps as having a closed incisura columellae auris enclosing both the stapes and Eustachian tube (see also character 54). However, in this species the closing of the incisura columellae auris is, according to all evidence, not homologous to that seen in other turtles. In Meiolania platyceps, the incisura is closed laterally by the extension of the quadratojugal and squamosal below the cavum tympani (Gaffney 1983: 415), not by the posteroventral surrounding of the columella auris by the quadrate as in other turtles. Hence, the extension of the quadratojugal and squamosal below the cavum tympani gives the impression that the incisura is closed in lateral view, but figures 38, 42 and 45 in Gaffney (1983) rather show an open incisura. According to these observations, Meiolania platyceps is tentatively scored state 0 in the present analysis. Taxa without incisura columellae auris are 
analysis. Taxa without incisura columellae auris are scored inapplicable for this character.

Character 54. Eustachian tube contained within the incisura columellae auris alongside the stapes: $0=$ absent; 1 = present (modified from Joyce 2007, ch. 36). See character 52 and 53 for a discussion. Taxa that have an open incisura columellae auris are scored unknown for this character.

Character 55. Epipterygoid: 0 = present; 1 = absent (Joyce 2007, ch. 37).

Character 56. Shape of the epipterygoid: $0=$ stout and pillar-shaped; 1 = laminar (modified from Sterli 2008, ch. 39). This character was proposed by Sterli (2008) following observations on Kayentachelys aprix by Sterli \& Joyce (2007). A stout, pillar-shaped epipterygoid (plesiomorphic amniote condition) is known in $K$. aprix and Meiolania platyceps (Gaffney 1983; Sterli \& Joyce 2007). This morphology was probably also present in Proganochelys quenstedti (Gaffney 1990). The condition in Mongolochelys efremovi is scored as unknown, but photographs of the PIN material provided by W. G. Joyce suggest that a similar morphology to that of Meiolania platyceps may have been present.

Character 57. Pterygoid teeth: $0=$ present: $1=$ absent (Joyce 2007, ch. 38). Based on Rougier et al. (1995), Joyce (2007) scored Palaeochersis talampayensis as possessing pterygoid teeth. However, according to a recent redescription of the material by Sterli et al. (2007), the structures described as denticles by Rougier et al. (1995) are artefacts. Consequently, and considering the relatively poor preservation of this area in available specimens (Sterli et al. 2007), Palaeochersis talampayensis is scored as unknown regarding this character.

Character 58. Basipterygoid articulation: 0 = open; 1 = fused (Joyce 2007, ch. 39).

Character 59. Interpterygoid vacuity: 0 = present; 1 = absent (Joyce 2007, ch. 40).

Character 60. Posterior process of the pterygoid that floors the cavum acustico-jugulare: $0=$ absent; $1=$ present (modified from Joyce 2007, ch. 31). See character 47 for a discussion. As preserved, the posterior process of the pterygoid in Chubutemys copelloi has a limited extent and does not contact the basioccipital posteriorly. However, according to Gaffney et al. (2007), the posterior margin of this process is broken so that it may have floored the cavum acustico-jugulare entirely and contacted the basioccipital. Consequently, Chubutemys copelloi is cautiously scored as unknown for characters 60 and 61 (see below).
Character 61. Pterygoid-basioccipital contact: $0=$ absent; 1 = present (Joyce 2007, ch. 41).

Character 62. Processus trochlearis pterygoideus: $0=$ absent; 1 = present (Joyce 2007, ch. 42).

Character 63. Foramen palatinum posterius: $0=$ present; 1 = absent (modified from Joyce 2007, ch. 43). Herein, the original unordered multistate character of Joyce (2007; foramen palatinum posterius present; open laterally; absent) is recoded as two separate binary characters (characters 63 and 64). This coding allows to test, by congruence, whether or not taxa that lack the foramen palatinum posterius derive from groups which are characterised by a laterally open foramen. A foramen palatinum posterius that is open laterally (character 64 ) is found only in Plesiochelys solodurensis, 'Thalassemys' moseri and Santanachelys gaffneyi, whereas the absence of the foramen palatinum posterius (character 63) characterises extant chelonioids. Joyce (2007) scored Sandownia harrisi as having a foramen palatinum posterius that is open laterally, but this does not appear on the published illustrations (Meylan et al. 2000). In contrast, Meylan et al. (2000) described the foramen palatinum posterius as being a narrow opening situated just lateral to the apertura narium interna, in which case the foramen would be entirely surrounded by bone in this species. The palatal region of Sandownia harrisi is actually highly modified because of the presence of a very extensive secondary palate, so that only a firsthand examination of the only known specimen could clarify this situation. In the meantime, Sandownia harrisi is scored unknown for characters 63, 64 and 66 of the present analysis. According to Rieppel (1993), during early stages of ossification of the palate, the foramen palatinum posterius of turtles appears in the same topological position as the suborbital fenestra of other reptiles. Following Laurin \& Reisz (1995) and Rieppel (1993, 1994: 44), the foramen palatinum posterius and suborbital fenestra are considered homologous herein. This allows the scoring of the outgroups for this character.

Character 64. Foramen palatinum posterius open laterally: 0 = absent; 1 = present (modified from Joyce 2007, ch. 43). See character 63 for a discussion. Taxa that have lost the foramen palatinum posterius are scored inapplicable for this character.

Character 65. Medial contact of pterygoid: $0=$ present, pterygoids in medial contact with one another for at least part of their length; 1 = absent, contact of the basisphenoid with the vomer and/or palatines present (Joyce 2007, ch. 44). Australochelys africanus is scored as unknown for this character (see character 44, above). In contrast to Joyce (2007; state 1), but following Sukhanov \& 
Narmandakh (1974) and Sukhanov (2000), Hangaiemys hoburensis is scored as having a medial contact between the pterygoids (state 0 ).

Character 66. Pterygoid contribution to foramen palatinum posterius: 0 = present; 1 = absent (Joyce 2007, ch. 45). Joyce (2007) scored Meiolania platyceps as polymorphic for this character. This is probably the result of the lack of precision of the illustrations published by Gaffney (1983) that sometimes represent the pterygoid forming part of the foramen palatinum posterius and sometimes not. However, Gaffney's (1983) description is less ambiguous and states that the pterygoid forms part of the foramen palatinum posterius in this species. Consequently, Meiolania platyceps is scored state 0 in the present analysis.

Character 67. Intrapterygoid slit: $0=$ absent; $1=$ present (Gaffney \& Meylan 1988, ch. C11-2; Gaffney 1996, ch. M13; Gaffney et al. 2007, ch. 38). The intrapterygoid slit is a feature unique to meiolaniids. It consists of a "thin sheet of bone formed by the pterygoids that encloses a space ventral to the basisphenoid" (Gaffney 1983: 429). It is found in Meiolania platyceps, Ninjemys oweni and Niolamia argentina.

Character 68. Crista supraoccipitalis: $0=$ poorly developed; 1 = protruding significantly posterior to the foramen magnum (Joyce 2007, ch. 46).

Character 69. Large supraoccipital exposure on dorsal skull roof: 0 = absent; 1 = present (Joyce 2007, ch. 47). According to Joyce (2007), a large supraoccipital exposure on the skull roof is present in Meiolania platyceps and Mongolochelys efremovi. However, as pointed out by Gaffney et al. (2007), the dorsal exposure of the supraoccipital is clearly not as well developed in Mongolochelys efremovi as it is in Meiolania platyceps. In the present analysis, only Meiolania platyceps and Ninjemys oweni are scored state 1, whereas Niolamia argentina is scored as unknown.

Character 70. Medial contact of exoccipitals dorsal to foramen magnum: 0 = absent; 1 = present (Joyce 2007, ch. 48).

Character 71. Processus paroccipitalis: $0=$ loosely articulated to squamosal and quadrate; 1 = tightly sutured to squamosal and quadrate (Joyce 2007, ch. 49).

Character 72. Rostrum basisphenoidale: 0 = flat; 1 = rod-like, thick and rounded (Joyce 2007, ch. 50).

Character 73. Paired pits on ventral surface of basisphenoid: 0 = absent; 1 = present (Joyce 2007, ch. 51).

Character 74. Basispterygoid process: $0=$ present; $1=$ absent (modified from Sterli 2008, ch. 41). Sterli (2008) proposed a modification of character 39 of Joyce (2007) in coding the absence/presence of a basipterygoid process along with the fusion of the basipterygoid articulation into a single multistate character. However, the absence/ presence of a basipterygoid process is probably an independent character, as suggested by the persistence of this process in some taxa with a fused basipterygoid articulation (e.g., Kayentachelys aprix, Condorchelys antiqua, Pleurosternon bullockii and Glyptops plicatulus). Consequently, the absence/presence of basipterygoid process is coded here as a separate character from the fusion of the basipterygoid articulation (see character 58, above).

Character 75. Position of the canalis stapediotemporalis: 0 = posterior to fenestra ovalis between the paroccipital process of the opisthotic and the quadrate; 1 $=$ anterior to fenestra ovalis between the quadrate and the prootic (Joyce 2007, ch. 53).

Character 76. Foramen stapedio-temporale: $0=$ present; 1 = absent (modified from Joyce 2007, ch. 54). Joyce (2007) coded the morphology of the foramen stapedio-temporale as an ordered multistate character (foramen relatively large; foramen significantly reduced; foramen absent). According to the coding strategy followed herein, this character is recoded as two separate binary variables (characters 76 and 77). These characters would then be tested for congruence along with other hypotheses of homology and the resulting topology would (ideally) indicate whether or not the loss of foramen stapedio-temporale in some taxa derives from a reduction of the size of this foramen in less inclusive clades, as suggested by Joyce's original assumption.

Character 77. Size of the foramen stapediotemporale: 0 = relatively large (the size of a blood foramen); 1 = significantly reduced in size (modified from Joyce 2007, ch. 54). See character 76 for a discussion. Taxa that have lost the foramen stapedio-temporale are scored inapplicable for this character.

Character 78. Foramen jugulare posterius formed by bone: 0 = absent; 1 = present, formed mostly by the exoccipital (Sterli 2008, ch. 62; modified from Joyce 2007, ch. 55).

Character 79. Foramen posterius canalis carotici interni formed by the prootic only: 0 = absent; 1 = present (modified from Joyce 2007, ch. 56). Characters related to the carotid arterial system have consistently been used by systematists in order to resolve relationships within turtles (e.g., Gaffney 1979). This is especially true with respect to the position of the foramen posterius canalis carotici interni (fpcci), the foramen through which the internal carotid artery enters the cranium. Four morphologies can be recognised among turtles (Gaffney 
1979). The plesiomorphic amniote condition (i.e., fpcci formed entirely within the basisphenoid) is found in most stem turtles, some panpleurodires and maybe also some pancryptodires (if these species are correctly interpreted as belonging to this clade). Most pleurodires show a condition where the fpcci is formed by the prootic only. Paracryptodires (i.e., baenids, pleurosternids and closely related species) have a fpcci that opens between the basisphenoid and pterygoid, halfway along the suture between these two bones. Finally, the fpcci of eucryptodires and Meiolania platyceps is formed mostly or fully by the pterygoid and is located near the posterior edge of this bone. The interpretation of potential homologies between these different morphologies have varied and the debate is not yet closed (see Jamniczky et al. 2006; Sterli et al. in press). As Notoemys laticentralis, the only stem pleurodire in which the skull is known, and some extant pleurodires (e.g., Erymnochelys madagascariensis, Podocnemis expansa) retain the plesiomorphic amniote condition (although not exactly as in basal turtles), it is reasonable to conclude that the condition where the fpcci opens within the prootic evolved independently within crown-group pleurodires. Concerning the two morphologies found within pancryptodires, it has been proposed that the paracryptodire condition was an intermediate state toward eucryptodire morphology (e.g., Evans \& Kemp 1976; Rieppel 1980; Gaffney \& Meylan 1988; Gaffney et al. 1991). However, available evidence suggests that the two conditions may have evolved separately from the plesiomorphic amniote condition or another ancestral morphology (Brinkman \& Nicholls 1993; Jamniczky et al. 2006). Consequently, and although it is obviously not a perfect answer to this problem, the solution to code this character complex into three binary characters, one representing each derived morphology (characters 79, 80 and 81 herein), appears to be less questionable in terms of primary homology assessments for the moment. This coding broadly corresponds to that used by Gaffney et al. (1991), Gaffney (1996) and Brinkman \& Wu (1999). In contrast, Dryden (1988), Hirayama et al. (2000), Joyce (2007) and Sterli et al. (2007) coded a single, unordered multistate character regrouping all of the four aforementioned morphologies. The latter coding is less appropriate because it assumes a priori that all morphologies are homologous to one another and that the transition from any derived state to the other is possible in theory and as likely to happen as the transformation from the plesiomorphic condition into one of the derived morphology (i.e., 1 step). In other words, homology assumptions between character states are made before the analysis and are impossible to test for congruence because they are locked into a single character. Chubutemys copelloi is scored unknown for characters 79, 80 and 81 because the exact position of the fpcci is not known in this species (Gaffney et al. 2007: 19).

Character 80. Foramen posterius canalis carotici interni positioned halfway along the suture between the basisphenoid and the pterygoid: 0 = absent; 1 = present (modified from Joyce 2007, ch. 56). See character 79 for a discussion.

Character 81. Foramen posterius canalis carotici interni formed by the pterygoid and positioned near the posterior edge of this bone: 0 = absent; 1 = present (modified from Joyce 2007, ch. 56). See character 79 for a discussion. Joyce (2007) scored Santanachelys gaffneyi as having a fpcci formed by the pterygoid (i.e., state 1), but the original description and illustrations of the only known specimen of this species (Hirayama 1998) indicate that the fpcci opens between the basisphenoid and pterygoid (i.e., the paracryptodire morphology; see character 80, above). Furthermore, Hirayama (1998: 707) stated that all protostegids are characterised by a fpcci opening between the basisphenoid and pterygoid. Known protostegid skulls are not currently well described in the literature, which prevents the resolution of this disagreement between Hirayama (1998) and Joyce (2007). In order to avoid favouring one interpretation or the other, Santanachelys gaffneyi is scored unknown for the present character as well as for characters 79 and 80 (see above). According to Sukhanov (2000: 314 and fig. 17.2), the fpcci opens within the basisphenoid and posteriorly "the internal carotid arteries are situated in an open ventral groove formed by the basisphenoid" in Annemys levensis, the only xinjiangchelyid for which a skull has been described. However, newly discovered material of this species indicates that the fpcci actually opens posteriorly in the pterygoid and that the floor of the canalis caroticus internus is not entirely ossified anteriorly, as in sinemydids for example (W. G. Joyce, pers. comm. 2010). Hence, Annemys levensis is scored state 1 for the present character.

Character 82. Fenestra perilymphatica: $0=$ large; $1=$ reduced in size to that of a small foramen (Joyce 2007, ch. 57). The fenestra perilymphatica is not defined by bone either in Proganochelys quenstedti or in Australochelys africanus (Gaffney 1990; Gaffney \& Kitching 1995), so that these taxa are scored inapplicable for this character. Similarly, all of the outgroups are scored inapplicable for this character.

Character 83. Medial contact of dentaries: 0 = fused; 1 = open suture (Joyce 2007, ch. 58). 
Character 84. Splenial: 0 = present; 1 = absent (Joyce 2007, ch. 59).

Character 85. Lateral exposure of angular: $0=$ exposed along one-third of the lateral surface of the posterior part of the mandible; 1 = exposed only as a small sliver (dorsoventrally short) or absent along the lateral surface of the mandible (Gauthier et al. 1988, ch. 99; deBraga \& Rieppel 1997, ch. 89; Müller, J. 2004, ch. 167; Hill 2005, ch. 180). This character is included for outgroup comparison. It is invariant among turtles, which all possess state 1. Among other amniotes, only the lepidosauromorphs (here Simosaurus gaillardoti and Gephyrosaurus bridensis) are known to have this derived condition (e.g., deBraga \& Rieppel 1997: 316). However, stem lepidosaurs appear to lack this feature, so the conditions in lepidosaurs and sauropterygians are probably independently acquired (S. E. Evans, pers. comm., 2009).

Character 86. Bony turtle shell (as described by Zangerl 1939, 1969; Gaffney \& Meylan 1988: 161): $0=$ absent; 1 = present (Dryden 1988, ch. 35; Gaffney \& Meylan 1988, ch. A4-1; Hill 2005, ch. 344; Sterli 2008, ch. 72). This character is included for outgroup comparison. All turtles, with the exception of Odontochelys semitestacea and Dermochelys coriacea, have a fully formed bony turtle shell, as described by Zangerl $(1939,1969)$ and Gaffney \& Meylan (1988). Because they do not have a turtle shell, all of the outgroups are scored inapplicable for the following characters related to this structure (i.e., characters 87 to 140, 153 to $155,163,168,170$ and 172).

Character 87. Carapacial scales: $0=$ present; $1=$ absent or nearly absent (modified from Joyce 2007, ch. 60). Joyce (2007) originally coded an ordered multistate character (carapacial scales present; partly present; absent), the intermediate state being attributed to Mesodermochelys undulatus, Pseudanosteira pulchra and Carettochelys insculpta. However, the carapacial scales are present in Pseudanosteira pulchra (e.g., Hay 1908; Clark 1932) and absent in Carettochelys insculpta (e.g., Bonin et al. 2006). With regard to these considerations, the present study follows Shaffer et al. (1997, ch. 78) in coding only the absence/presence of scales on the carapace. The hypothesised gradual loss of carapacial scales in relatives of Dermochelys coriacea and in Trionychia would necessitate a broader taxon sampling to be tested. The condition in Odontochelys semitestacea is unknown ( $\mathrm{Li}$ et al. 2008).

Character 88. Tricarinate carapace: 0 = absent; $1=$ present (modified from Joyce 2007, ch. 61). Joyce (2007: 28) coded an ordered multistate character for this feature (absent; present, but only poorly developed; present and pronounced). According to the coding strategy followed herein, this character should be recoded as two separate binary variables. However, given that I did not have access to the relevant material (mainly extant kinosternoids), I found it difficult to reproduce the 'poorly developed' state observed by Joyce. Consequently, the present study follows the absent/present coding of Meylan \& Gaffney (1989, ch. 19) and Shaffer et al. (1997, ch. 93).

Character 89. Articulation of nuchal with neural spine of eighth cervical vertebra along a blunt facet: $0=$ present; 1 = absent (modified from Joyce 2007, ch. 62). All turtles, with the exception of stem turtles and panchelonioids, lack an articulation between the eighth cervical vertebra and the nuchal plate. In stem turtles (e.g., Proganochelys quenstedti, Kayentachelys aprix and Mongolochelys efremovi), the articulation is formed along a blunt facet on the visceral surface of the nuchal. In contrast, the eighth cervical vertebra of panchelonioids articulates with a raised pedestal formed on the ventral surface of the nuchal. According to available evidence, these two morphologies are not homologous (e.g., Joyce 2007: 29) and should consequently be coded as two separate characters (characters 89 and 90).

Character 90. Raised pedestal on the visceral surface of the nuchal for the articulation with the neural spine of the eighth cervical vertebra: $0=$ absent; $1=$ present (modified from Joyce 2007, ch. 62).

Character 91. Elongate costiform processes of the nuchal: $0=$ absent; 1 = present, process crosses peripheral 1 to contact peripheral 2 and sometimes peripheral 3 (Joyce 2007, ch. 63).

Character 92. Neural formula $6>4<6<6<6<6$ : $0=$ absent; 1 = present (Joyce 2007, ch. 64).

Character 93. Neurals: $0=$ present; $1=$ absent, allowing medial contact of all costals (modified from Joyce 2007 , ch. 68). Joyce (2007) coded the absence/presence of medial contact between costal bones as an ordered multistate character (absence of medial contact; medial contact of up to three posterior costals; medial contact of all costals, neurals absent). This coding suggests that the condition where all neurals are missing, which is found only in some chelids (only in Chelodina oblonga and Elseya dentata herein), evolved from a condition where only some posterior neurals were absent, allowing a partial contact between posterior costals. The latter condition is found in all other extant pleurodires included in the present analysis, but also in numerous pancryptodires (see character 94). Although the aforementioned assumption regarding the origin of the condition in some chelids is probably correct, the only way to test it through the analysis is to code two binary characters. In the 
A

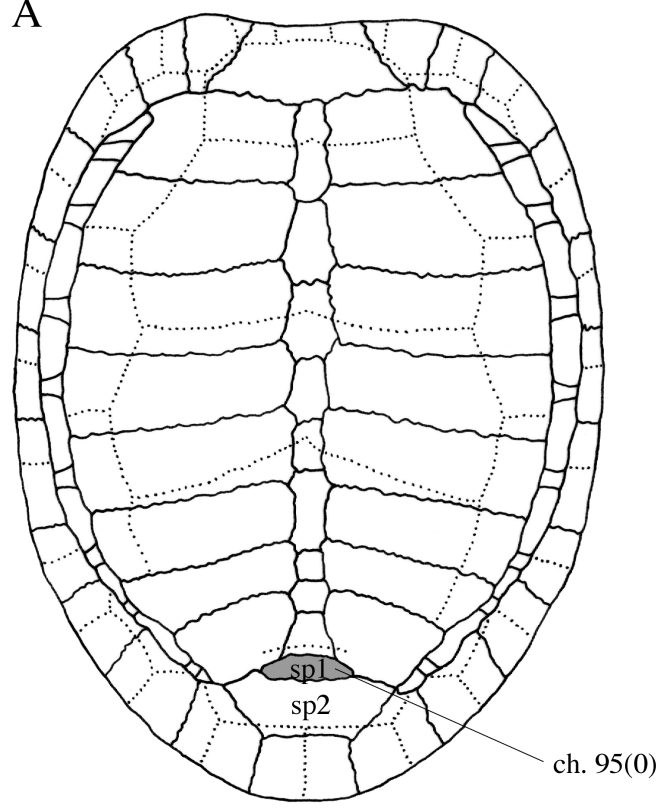

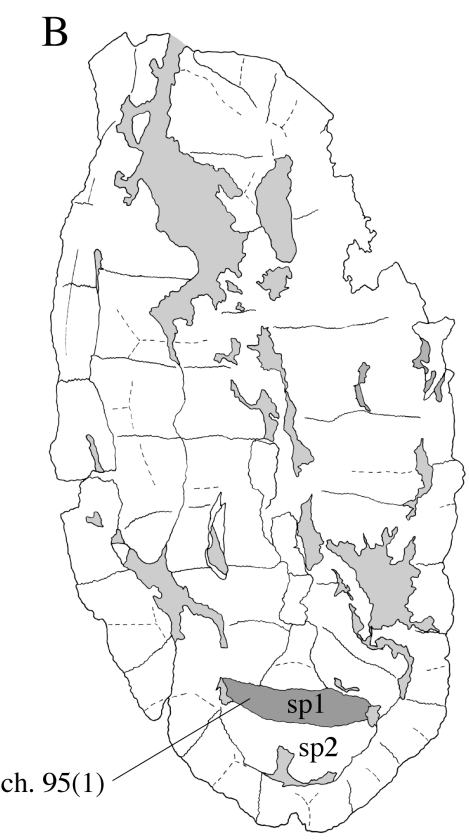

Figure 3- Illustration of states for character 95. (A) reconstruction of the carapace of Heckerochelys romani, redrawn from Sukhanov (2006); (B) carapace of Eileanchelys waldmani, specimen NMS G 2004.31.16b (see Anquetin et al. 2009). Abbreviation: sp, suprapygal.

present study, the complete absence of neurals (character 93) is coded separately from the partial reduction of posterior neurals (character 94).

Character 94. Partial reduction of the posterior neurals allowing medial contact of up to three posterior costals: 0 = absent; 1 = present (modified from Joyce 2007, ch. 68). See character 93 for a discussion. A partial reduction of posterior neurals allowing medial contact of posterior costals is found in numerous extant pleurodires as well as in eleven of the pancryptodiran taxa included in the present analysis (see the matrix in the Supplementary Material). The present study differs from that of Joyce (2007; states 0 and 1, respectively) in the scoring of Mesodermochelys undulatus and Peltochelys duchastelli. In $M$. undulatus the posterior neurals are reduced and allow a medial contact of some posterior costals (Hirayama \& Chitoku 1996), whereas $P$. duchastelli has a complete row of neural that prevents any medial contact of costals (Meylan 1988).

Character 95 (Fig. 3). Lateral contact of suprapygal 1 with peripherals: $0=$ absent; 1 = present (new character). In most turtles, the first suprapygal does not contact the peripherals laterally. A contact between the first suprapygal and the peripherals is present in Eileanchelys waldmani, Kallokibotion bajazidi, Naomichelys speciosa, Plesiochelys solodurensis and Xinjiangchelys latimarginalis.

Character 96. Peripherals: $0=$ present; $1=$ absent (modified from Joyce 2007, ch. 65). Joyce (2007) coded the number of peripherals into a four-state ordered character (more than 11 pairs; 11 pairs; 10 pairs; less than 10 pairs). According to the coding strategy followed herein, the absence/presence and the number of peripherals are coded as separate variables (characters 96, 97 and 98). Hence, the present study partly follows the coding of Shaffer et al. (1997, chs 83, 95).

Character 97. Number of peripherals: $0=$ more than 11 pairs; $1=11$ pairs or less (modified from Joyce 2007, ch. 65). See character 96 for a discussion. Taxa that lack peripherals are scored inapplicable for this character.

Character 98. Number of peripherals: $0=11$ pairs or more; 1 = reduced to 10 pairs (modified from Joyce 2007, ch. 65). See characters 96 and 97 for a discussion. Taxa that lack peripherals are scored inapplicable for this character.

Character 99. Anterior peripherals incised by musk ducts: 0 = absent; 1 = present (Joyce 2007, ch. 66).

Character 100. Medial contact of the first pair of costals: 0 = absent; 1 = present, but complete set of neurals present (Joyce 2007, ch. 67).

Character 101. Reduction of costal ossification: $0=$ absent, costals fully or almost fully ossified, costal fontanelles small or absent; 1 = present, costals ossified only two thirds the length of the costal ribs, costal fontanelles well developed (Joyce 2007, ch. 69).

Character 102. Cervical: $0=$ present; $1=$ absent, carapacial scales otherwise present (modified from Joyce 2007 , ch. 70). Joyce (2007) coded an unordered multistate 
character for the absence/presence and number of cervical scales (one cervical scale; cervical scale absent; more than one cervical scale). According to the methodology of character construction followed herein, the absence/presence of cervical scales and the number of these scales are considered as two separate variables (characters 102 and 103). According to Milner (2004) and personal observations (e.g., NHM 28618, 38733, 43621, R3413, R3727 and R 6895), Pleurosternon bullockii is scored as lacking a cervical scale, which contrasts with Joyce (2007).

Character 103. Number of cervical scales: $0=$ one cervical scale; $1=$ more than one cervical scale present (modified from Joyce 2007, ch. 70). Taxa that lack a cervical scale are scored inapplicable for this character.

Character 104. Marginal scales overlap onto costal plates: $0=$ absent, marginals restricted to peripheral plates; 1 = present (Matzke et al. 2004, ch. 7; Danilov \& Parham 2008, ch. Marginal B; see also Meylan \& Gaffney 1989, ch. 47, and Peng \& Brinkman 1993). In the context of the present analysis, marginal scales overlapping onto costal bones are known in pleurosternids, Xinjiangchelys latimarginalis, $X$. qiguensis and Adocus beatus.

Character 105. Supramarginals: $0=$ present; $1=$ absent (modified from Joyce 2007, ch. 71). Joyce (2007) coded the absence/presence of supramarginal scales as an ordered multistate character (complete row present; partial row; supramarginals absent). According to Joyce (2007), Proterochersis robusta, Platychelys oberndorferi and Macrochelys temminckii have a partial row of supramarginals, whereas Proganochelys quenstedti and Palaeochersis talampayensis have a complete row of supramarginals. In their recent redescription of Palaeochersis talampayensis, Sterli et al. (2007) showed that it is unknown whether a complete or partial row of supramarginals was present: only three supramarginals are visible on each sides of the only available shell of this species. This contrasts with the coding of Joyce (2007). Proganochelys quenstedti is consequently the only species with a complete row of supramarginals and it is impossible to know whether this is a plesiomorphic or autapomorphic condition (uninformative). Following these observations, the coding of a simple absent/ present character for the supramarginals appears to be more appropriate for the moment. The number of supramarginals in Macrochelys temminckii appears to be highly variable (e.g., Bonin et al. 2006) and these additional scales may actually be absent in some specimens, according to personal observation (FMNH dried skeleton collection). Nevertheless, M. temminckii is conservatively scored state 0 in the present analysis. According to personal observation of BSPG AS I 1438, the presence of a partial row of supramarginals in Platychelys oberndorferi is dubious. This specimen appears to have only one supernumerary scale that is located anterior to the first pleural. This observation is confirmed by illustrations of the lost holotype of this species published in Meyer (1860: pl. XVIII.4) and Wagner (1861: taf. IV). Whether this additional scale corresponds to a true supramarginal or to the supernumerary pleural scale found anterior to the first pleural in Palaeomedusa testa, Thalassemys marina and Caretta caretta (see Joyce 2003, 2007) remains unknown. Joyce (2003: 6) considered this additional scale in Platychelys oberndorferi to be a supernumerary pleural, whereas Joyce (2007: 33) interpreted it as a supramarginal. Consequently, Platychelys oberndorferi is scored as unknown for this character. Finally, Boremys pulchra is scored as having supramarginals (Gaffney 1972b; Lyson \& Joyce 2009).

Character 106. Shape of vertebrals: $0=$ vertebrals 2 to 4 significantly broader than pleurals; 1 = vertebrals 2 to 4 as narrow as, or narrower than, pleurals (Joyce 2007, ch. 73). Palaeochersis talampayensis is scored as unknown for this character because the vertebrals are not apparent in available specimens (Rougier et al. 1995; Sterli et al. 2007). This scoring differs from that of Joyce (2007; state $0)$.

Character 107. Position of vertebral 3-4 sulcus in taxa with five vertebrals: 0 = sulcus positioned on neural $6 ; 1=$ sulcus positioned on neural 5 (Joyce 2007, ch. 74). Although the vertebral 3-4 sulcus crosses neural 5 in the published reconstruction of Heckerochelys romani, the original description and photographs fail to confirm this characteristic (Sukhanov 2006). Considering the fact that most basal taxa have a vertebral 3-4 sulcus on neural 6, Heckerochelys romani is cautiously scored as unknown for this character.

Character 108 (Fig. 4). Vertebral 3-4 sulcus with a wide medial embayment oriented posteriorly: 0 = absent; 1 = present (new character). A wide, U-shaped medial embayment of the vertebral 3-4 sulcus oriented posteriorly is known in Chengyuchelys baenoides, $C$. zigongensis and Annemys levensis (Young \& Chow 1953; Ye 1982, 1994; Sukhanov 2000; Sukhanov \& Narmandakh 2006; Fig. 4B). It is also present in Annemys latiens, which is not included in the present analysis (see Sukhanov 2000).

Character 109. Vertebral 5: $0=$ overlaps onto peripherals and often pygal posteriorly; 1 = does not overlap onto peripherals and pygal (modified from Matzke et al. 2004, ch. 6). Matzke et al. (2004) used this character in their analysis of xinjiangchelyid interrelationships. According to these authors, most xinjiangchelyids can be distinguished from other turtles 
A

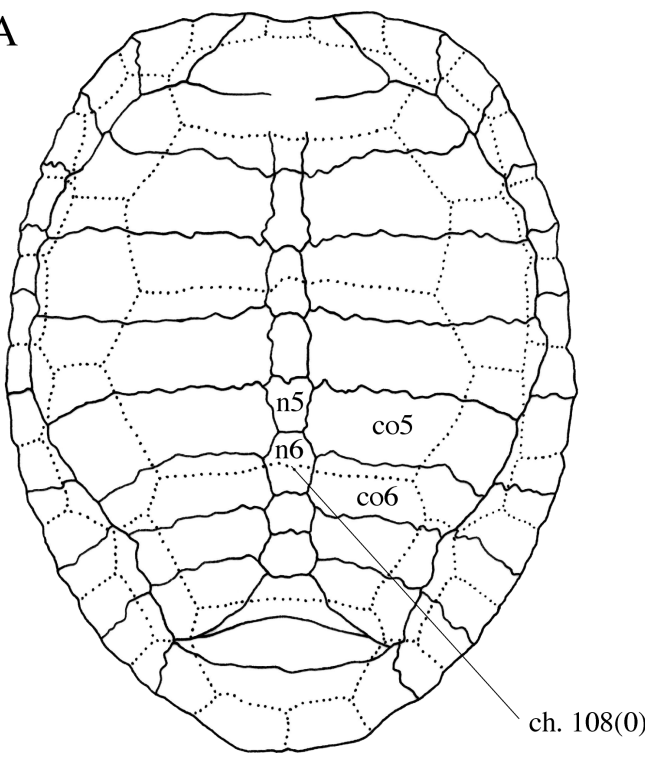

B

ch. 108(1)

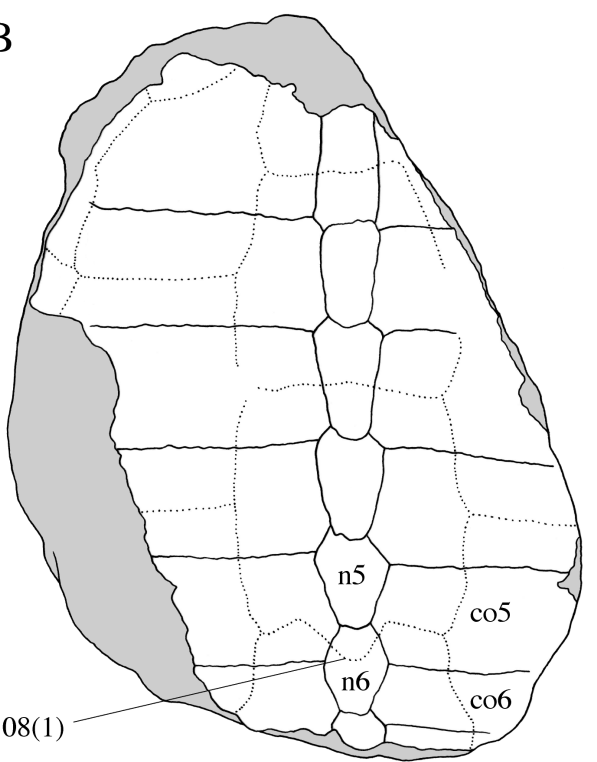

Figure 4 - Illustration of states for character 108. (A) reconstruction of the carapace of Dinochelys whitei, redrawn from Gaffney (1979a); (B) carapace of Chengyuchelys baenoides, specimen IVPP V708 (cast of the holotype). Abbreviations: co, costal plate; n, neural plate.

by a fifth vertebral scale that does not overlap, or only slightly overlaps, the peripherals. However, the broader taxon sample considered in the present analysis shows that vertebral 5 usually overlaps the peripherals only to a limited extent in most turtles. With regards to these observations, the character has been redefined herein so that the supposed derived state is restricted to those taxa in which the fifth vertebral does not overlap either the peripherals or the pygal. Following this new definition, state 1 is present only in Xinjiangchelys latimarginalis and $X$. tianshanensis among xinjiangchelyids. However, this state is also found in Mongolochelys efremovi, Pleurosternon bullockii, Glyptops plicatulus, Naomichelys speciosa, Siamochelys peninsularis, Zangerlia neimongolensis and Adocus beatus.

Character 110. Connection between carapace and plastron: 0 = osseous; 1 = ligamentous (Joyce 2007, ch. 75). Odontochelys semitestacea is scored as unknown because there is no evidence as to whether or not a connexion was present between the plastron and the almost non-existent carapace of this species ( $\mathrm{Li}$ et al. 2008). Xinjiangchelys qiguensis is also scored as unknown for this character because the original description of Matzke et al. (2004) is unclear on that particular matter.

Character 111. Central plastral fontanelle: $0=$ absent in adult individuals; 1 = present, even in adult individuals (Joyce 2007, ch. 76). In their reconstruction of Indochelys spatulata, Datta et al. (2000: fig. 5) depicted a plastron without a fontanelle. However, the central plastral area of the only known specimen of this species is preserved as a gaping hole, which prevents any conclusion on the absence or presence of a central plastral fontanelle (Datta et al. 2000: fig. 3). Consequently, Indochelys spatulata is scored as unknown for this character. In her reassessed version of Joyce's (2007) matrix, Sterli (2008) scored Condorchelys antiqua as having a central plastral fontanelle. I follow this scoring in the present analysis, but it should be noted that the presence of a central plastral fontanelle in C. antiqua is only tentatively proposed (Sterli \& de la Fuente 2010). Although the presence of a fontanelle may be a true synapomorphy for some clades (e.g. panchelonioids), the fact that fontanelles are very commonly present in young individuals and that it is apparently easy for aquatic species to retain this feature in adult individuals suggest that this character should be used carefully or even excluded from future phylogenetic analyses. Many fossil species are known by a limited number of individuals so that the conclusion that a fontanelle is present in adult individuals is often questionable. Eileanchelys waldmani is a perfect illustration of this phenomenon: a central plastral fontanelle was apparently present in one individual but it is entirely absent in other specimens (Anquetin et al. 2009; Anquetin in press). Not only that this can lead to inaccurate scoring of some species, but it can result in unwarranted grouping of some species, as it may be the case for Heckerochelys romani and Condorchelys antiqua in the present study (see below).

Character 112. Plastral kinesis: $0=$ absent, scale sulci and bony sutures do not overlap; 1 = present, scale sulci 
coincide with epiplastral-hyoplastral contact (Joyce 2007, ch. 77).

Character 113. Anterior entoplastral process: $0=$ present, medial contact of epiplastra absent; 1 = absent, medial contact of epiplastra present (Joyce 2007, ch. 78). Sterli (2008) scored Condorchelys antiqua as having an anterior process of the entoplastron, however in the description of this species she states that the epiplastra actually meet medially (ibid.: 288). Consequently, Condorchelys antiqua is scored state 1 in the present analysis.

Character 114. Size of the posterior entoplastral process: 0 = posterior process long, reaching as far posteriorly as the mesoplastra; 1 = posterior process reduced in length (Joyce 2007, ch. 79).

Character 115. Entoplastron: 0 = massive and crossto diamond-shaped; 1 = strap like and V-shaped (Joyce 2007, ch. 81).
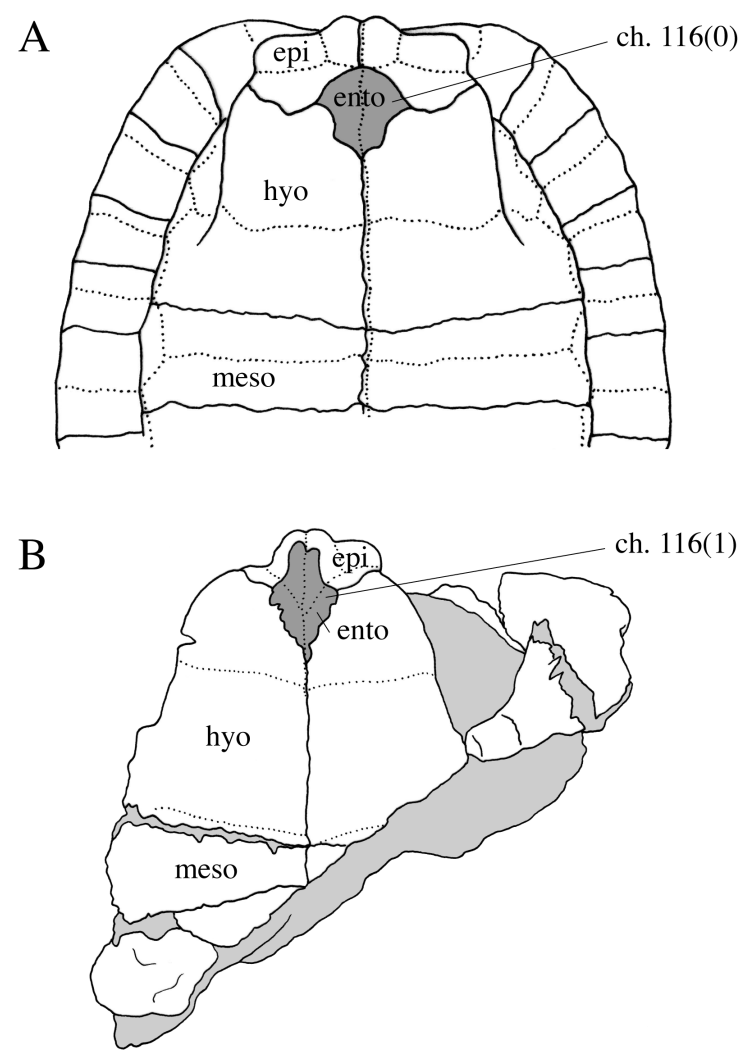

Figure 5 - Illustration of states for character 116. (A) reconstruction of the plastron of Dinochelys whitei, redrawn from Gaffney (1979a); (B) plastron of Chengyuchelys baenoides, specimen IVPP V6507. Abbreviations: ento, entoplastron; epi, epiplastron; hyo, hyoplastron; meso, mesoplastron.

Character 116 (Fig. 5). Dagger-shaped entoplastron: 0 = absent; 1 = present (new character). Chengyuchelys baenoides and $C$. zigongensis are unique among turtles in having an entoplastron shaped like a short dagger (e.g., Danilov \& Parham 2008: fig. 2B; these authors described the entoplastron as being leaf-shaped). Anteriorly, the entoplastron has an anterior process, that is probably not homologous to the anterior entoplastral process of more basal turtles (see character 113), which corresponds to the 'handle' of the dagger. At the level of the medialmost point of the epiplastron-hyoplastron suture, the entoplastron has a short lateral projection on each side that recalls the 'guard' of the dagger. Finally, the posterior part of the entoplastron tapers progressively posteriorly, corresponding to the shortened blade of the imaginary dagger. The posterolateral margins of the entoplastron are not straight but rather slightly sinuous.

Character 117. Entoplastron: $0=$ present; 1 = absent (Joyce 2007, ch. 82).

Character 118. Shape and contact of epiplastra: $0=$ epiplastra sub-square in outline, minor posterior contact with hyoplastra; 1 = epiplastra elongate in shape, long posteromedial contact with hyoplastra (Joyce 2007, ch. 83). Based on personal observation of dried skeletons at the Field Museum (e.g., FMNH 51627), Platysternon megacephalum is scored state 1 in the present analysis, in contrast to Joyce (2007; state 0). Although the elongate shape of the epiplastra in $P$. megacephalum is less pronounced than that of Protochelydra zangerli or Chelydra serpentina, it is similar to the condition in Meiolania platyceps, Mongolochelys efremovi and Hangaiemys hoburensis, which are all scored state 1 in both Joyce (2007) and the present study.

Character 119. Contacts of axillary buttresses: $0=$ peripherals only; 1 = peripherals and first costal (Joyce 2007, ch. 84). Siamochelys peninsularis is scored as unknown because Tong et al. (2002: 691) expressed doubts on whether or not the axillary buttress contacts the first costal in this species.

Character 120. Mesoplastron: 0 = present; 1 = absent (modified from Joyce 2007, ch. 85; Sterli et al. 2007, ch. 70). Joyce (2007: 38) coded the number, development and presence of mesoplastra as an ordered multistate character (one or two pairs present; one pair of mesoplastra not meeting in midline; mesoplastra absent). Sterli et al. (2007: 59) followed a similar approach, although they scored the presence of two pairs of mesoplastra as a separate state (which was uninformative in their analysis) and ran the character unordered. Following the character construction methodology followed here, the absence/presence, the number, and the development of mesoplastra are kept as separate characters so that each of them constitutes a potential synapomorphy to be tested for congruence 
against other characters included in the analysis. By coding the absence/presence of mesoplastra as a simple binary character, the present analysis follows the majority of previous cladistic analyses of turtle relationships (Dryden 1988, ch. 44; Gaffney et al. 1991, ch. 31; Gaffney 1996, ch. 34; Brinkman \& Wu 1999, ch. 34; Hirayama et al. 2000, ch. 74; Gaffney et al. 2007, ch. 103). Siamochelys peninsularis was reported to have one pair of mesoplastra meeting medially (Tong et al. 2002), but I. G. Danilov (in Danilov \& Parham 2008) seriously questioned the presence of mesoplastra after seeing the few known specimens of this species. In order to avoid favouring one hypothesis or the other, Siamochelys peninsularis is scored as unknown for characters 120 to 122 .

Character 121. Mesoplastron: 0 = one pair present; 1 = two pairs present (modified from Joyce 2007, ch. 85; Sterli et al. 2007, ch. 70). Until the discovery of Odontochelys semitestacea (Li et al. 2008), Proterochersis robusta was the only turtle known to have two pairs of mesoplastra. Consequently, coding the number of mesoplastra as a separate character would have been uninformative. Following the discovery of Odontochelys semitestacea, the present analysis is the first to consider the number of mesoplastra as a separate character. The presence of two pairs of mesoplastra in Odontochelys semitestacea, arguably the most basal turtle known to date (Li et al. 2008; Reisz \& Head 2008), could have dramatic implications for the relationships of basalmost turtles: it suggests that the presence of two pairs of mesoplastra may be the plesiomorphic condition for turtles. Taxa in which the mesoplastra are lost are scored inapplicable for this character.

Character 122. Medial contact of mesoplastra: $0=$ present, or virtually present when a central plastral fontanelle is present, absence of contact between hyoplastron and hypoplastron; 1 = absent, partial contact between hyoplastron and hypoplastron present (modified from Gaffney et al. 1991, ch. 30; Joyce 2007, ch. 85; Sterli et al. 2007, ch. 70). Previous analyses that considered the absence/presence of a medial contact between the mesoplastra made no distinction between the condition in which mesoplastra are reduced and restricted to the lateral part of the plastron, hence allowing a broad medial contact between hyoplastra and hypoplastra, and the condition in which the mesoplastra do not meet at the midline because of the presence of a central plastral fontanelle, in which case there is no contact between hyoplastra and hypoplastra (Gaffney et al. 1991, ch. 30; Joyce 2007, ch. 85; Sterli et al. 2007, ch. 70). The definition of the present character is intended so that only taxa with reduced mesoplastra that actually do not contact each other medially and allow a contact between hyoplastra and hypoplastra are scored state 1 . It is assumed that species in which the mesoplastra reach the central plastral fontanelle without significant medial tapering would have presented a median contact of these plates if the fontanelle was closed (e.g., Heckerochelys romani and Mongolochelys efremovi). These species are consequently scored state 0 in the present analysis. According to this new definition, the absence of medial contact of the mesoplastra is exclusively found in Kallokibotion bajazidi and Panpleurodires, with the exception of chelids that have lost the mesoplastra. Joyce (2007) scored Baena arenosa as lacking a medial contact of the mesoplastra. Indeed, Gaffney (1972b: fig. 31) depicted a shell of a juvenile specimen of Baena arenosa in which the mesoplastra are reduced and do not contact one another medially. However, he clearly stated in the caption of this figure that "mesoplastra meet in midline in all other known B. arenosa shells" (Gaffney 1972b: 282). Baena arenosa is consequently scored state 0 in the present analysis. Taxa in which the mesoplastra are lost are scored as inapplicable for this character.

Character 123. Contacts of inguinal buttresses: $0=$ peripherals only; 1 = peripherals and costals (modified from Joyce 2007, ch. 86). Joyce (2007) followed Shaffer et al. (1997, ch. 55) in coding an unordered multistate character for the contacts of the inguinal buttress (contact with peripherals only; peripherals and costal 5; peripherals, costal 5 and costal 6). However, no taxon appears to possess the third state either in the description of the character, or in the matrix (Joyce 2007: 39 and 90-91). Consequently, the binary coding used by Dryden (1988, ch. 47), Hirayama et al. (2000, ch. 66) and Sterli et al. (2007, ch. 66) appears to be more appropriate.

Character 124. Distinct anal notch: $0=$ absent; $1=$ present (Joyce 2007, ch. 87). In contrast to Joyce (2007; state 0), Pleurosternon bullockii is scored state 1 because all specimens at the NHM (including the holotype NHM $\mathrm{R} 911$ ) in which this area of the plastron is preserved have distinct processes of the xiphiplastra that frame an anal notch. The present study also differs from that of Joyce (2007; state 1) in scoring Mongolemys elegans and Chrysemys picta as lacking an anal notch (state 0).

Character 125. Shape of xiphiplastra: $0=$ elongate rectangles; 1 = narrow struts that frame a xiphiplastral fontanelle (Joyce 2007, ch. 88).

Character 126. Plastral scales: $0=$ present; $1=$ absent (Joyce 2007, ch. 89).

Character 127. Midline sulcus of plastral scales: $0=$ straight; 1 = distinctly sinuous, at least for part of its length (Matzke et al. 2004, ch. 14; Joyce 2007, ch. 90; see also Peng \& Brinkman 1993). A sinuous midline sulcus of the plastral scales is known in Xinjiangchelys latimarginalis, 
X. tianshanensis, X. qiguensis, Siamochelys peninsularis, Annemys levensis, Zangerlia neimongolensis, Basilemys variolosa, Adocus beatus and Peltochelys duchastelli.

Character 128. Plastral scale set 1 , gulars: $0=$ one medially situated pair of scales present; 1 = one medially situated single scale present (Joyce 2007, ch. 91).

Character 129. Plastral scale set 1, gulars: $0=$ extending posteriorly onto entoplastron; 1 = limited to epiplastra only (modified from Matzke et al. 2004, ch. 15). In most turtles, the gulars extend posteriorly onto the entoplastron. In contrast, the gulars are limited to the epiplastra and do not extend posteriorly onto the entoplastron in Mongolochelys efremovi, 'Chengyuchelys' dashanpuensis, Siamochelys peninsularis, Xinjiangchelys latimarginalis, X. tianshanensis, X. qiguensis and Annemys levensis.

Character 130. Plastral scale set 2, extragulars: $0=$ present; 1 = absent (Joyce 2007, ch. 92).

Character 131. Medial contact of plastral scale set 2, extragulars: 0 = absent; 1 = present, extragulars contacting one another posterior to gulars (modified from Joyce 2007, ch. 93). Joyce (2007) coded an unordered multistate character for the absence/presence of a medial contact of the extragulars (medial contact absent; present, anterior to gulars; present, posterior to gulars). Within the context of the present analysis, as well as in that of Joyce (2007), a medial contact of the extragulars anterior to the gulars is only known in Chelodina oblonga and is consequently uninformative. Future studies should consider the medial contact of extragulars anterior to the gulars as a separate character.

Character 132. Anterior plastral tuberosities: $0=$ present; 1 = absent (Joyce 2007, ch. 94).

Character 133. Plastral scale set 8 , intergulars: $0=$ absent; 1 = present (Joyce 2007, ch. 95).

Character 134. Plastral scale set 3, humerals: $0=$ one pair present; 1 = two pairs present, subdivided by $\mathrm{a}$ plastral hinge (Joyce 2007, ch. 96).

Character 135. Plastral scale set 4, pectorals: $0=$ present; 1 = absent (Joyce 2007, ch. 97).

Character 136. Plastral scale set 5, abdominals: $0=$ present; 1 = absent (modified from Joyce 2007, ch. 98). Joyce (2007, ch. 98), following Meylan \& Gaffney (1989, ch. $34)$, coded both the absence/presence of abdominals and their medial contact with one another as an ordered multistate character (present, in medial contact; present, medial contact absent; abdominals absent). According to the coding strategy followed herein, these are kept as separate variables. The present coding corresponds to that of character 94 of Shaffer et al. (1997).
Character 137. Plastral scale set 5, abdominals: $0=$ contact one another medially; 1 = absence of medial contact between abdominals (modified from Joyce 2007, ch. 98). See character 136 for a discussion. The present coding corresponds to that of character 41 of Brinkman \& Wu (1999). Taxa that have lost abdominals are scored inapplicable for this character.

Character 138. Plastral scale set 7, anals: $0=$ only cover parts of the xiphiplastra; 1 = overlap anteromedially onto the hypoplastra (Joyce 2007, ch. 99).

Character 139. Inframarginal scales: 0 = present; 1 = absent (modified from Joyce 2007, ch. 100). The presence and number of inframarginals has been variously discretized as a character by successive authors. Dryden (1988, ch. 46) simply coded the inframarginals as absent/ present. Meylan \& Gaffney (1989, ch. 35) considered the number of inframarginals (five to four; four to three; three to two), with obvious problems regarding the necessary mutual exclusivity between character states. Shaffer et al. (1997, ch. 101) coded only the absence/ presence of a complete row of inframarginals, without differentiating the condition in panpleurodires where the inframarginals are lost and the condition in testudinoids where two pairs of inframarginals (axillaries and inguinals) are present. Hirayama et al. (2000, ch. 65) attempted to unify previously proposed codings into a multistate character ( 4 to 3 pairs; 2 pairs, axillaries and inguinals; inframarginals absent). Joyce (2007, ch. 100) broadly followed this latter proposition in coding an unordered multistate character (more than two pairs, complete row of inframarginals; two pairs present, limited contact between plastral scales and marginals; inframarginals absent, unlimited contact between plastral scales and marginals). In the present analysis, the absence/presence and number of inframarginals are coded as separate variables (characters 139 and 140).

Character 140. Inframarginals scales: $0=$ complete row present; 1 = only two pairs present (axillary and inguinal), limited contact between plastral scales and marginals present (modified from Joyce 2007, ch. 100). See character 139 for a discussion. Taxa that have lost the inframarginals are scored inapplicable for this character.

Character 141. Cervical ribs: $0=$ large cervical ribs present; 1 = cervical ribs reduced or absent (Joyce 2007, ch. 101). Joyce (2007) scored Palaeochersis talampayensis as having large cervical ribs, but the recent redescription of the material by Sterli et al. (2007) indicates that no specimen has cervical ribs preserved. Consequently, $P$. talampayensis is scored as unknown in the present analysis. 
Character 142. Position of the transverse processes: 0 = middle of the centrum; 1 = anterior end of the centrum (Joyce 2007, ch. 102).

Character 143. Posterior cervicals with strongly developed ventral keels: $0=$ absent; $1=$ present (Joyce 2007, ch. 103).

Character 144. Eighth cervical centrum significantly shorter than seventh: 0 = absent; 1 = present (Joyce 2007, ch. 104).

Character 145. Central articulations of cervical vertebrae: 0 = articulations not formed, cervical vertebrae amphicoelous or platycoelous; 1 = articulations formed, cervical vertebrae procoelous or opisthocoelous (Joyce 2007, ch. 105).

Character 146. Articulation between the centra of the second and third cervicals: $0=2(3 ; 1=2) 3$ (Joyce 2007, ch. 106). Characters 146 to 151 code for the shape of the articulation between successive centra of cervical vertebrae. Articular arrangements are given following the notation of Walther (1922) as implemented by Joyce (2007). This notation imitates the shape of the articular surfaces: the articulation between the centrum of the second cervical vertebra and that of the third is either convex anteriorly [i.e., 2(3] or concave anteriorly [i.e., 2)3]. Taxa that have amphicoelous or platycoelous cervical vertebrae are scored as inapplicable for these characters.

Character 147. Articulation between the centra of the third and fourth cervicals: $0=3(4 ; 1=3) 4$ (Joyce 2007, ch. 107).

Character 148. Articulation between the centra of the fourth and fifth cervicals: $0=4(5 ; 1=4) 5$ (Joyce 2007, ch. 108).

Character 149. Articulation between the centra of the fifth and sixth cervicals: $0=5(6 ; 1=5) 6$ (Joyce 2007, ch. 109).

Character 150. Articulation between the centra of the sixth and seventh cervicals: $0=6(7 ; 1=6) 7$ (Joyce 2007, ch. 110).

Character 151. Articulation between the centra of the seventh and eighth cervicals: $0=7(8 ; 1=7) 8$ (Joyce 2007, ch. 111).

Character 152. Articulation between the eighth cervical and the first dorsal vertebrae: $0=$ along the vertebral centra and zygapophyses; 1 = along zygapophyses only (modified from Joyce 2007, ch. 112). Joyce (2007) coded an unordered multistate character for the articulation between the eighth cervical and first dorsal vertebrae (8(dorsal; 8)dorsal; contact only at zygapophyses). The majority of turtles have an '8)dorsal' articulation, whereas in tryonichids the vertebrae only meet along the zygapophyses (ibid.: 45). Adocus beatus is unique among turtles in having an '8(dorsal' articulation and this state is uninformative in the context of the present analysis. Consequently, the character has been redefined to focus only on the loss of central vertebral articulation in trionychids.

Character 153. Length of the first dorsal rib: $0=$ long, extends full length of the first costal and may even contact the peripherals distally; $1=$ short, extends less than halfway across the first costal (modified from Dryden 1988, ch. 37; Gaffney et al. 1991, ch. 35; Rougier et al. 1995, ch. 49; Gaffney 1996, ch. 32; Brinkman \& Wu 1999, ch. 32; Hirayama et al. 2000, ch. 45; Gaffney et al. 2007, ch. 87; Joyce 2007, ch. 113; Sterli et al. 2007, ch. 72). The first dorsal rib of turtles does not fuse to the costal bones in contrast to the other dorsal ribs, but simply extends over the visceral surface of the first costal. In stem turtles, as well as numerous basal crown-group species, the first dorsal rib is of similar length to the remaining dorsal ribs, extending the full length of the first costal and often contacting the peripherals laterally. In more derived turtles, including all extant forms, the first dorsal rib is considerably reduced in length and extends less than halfway across the first costal. However, in some taxa (e.g., Platychelys oberndorferi and some baenids), the morphology is somewhat complicated by the development of a large axillary buttress that prevents the first dorsal rib from extending the full length of the first costal bone, despite the fact that the rib is well developed. Joyce $(2007$, ch. 113) tried to encompass the latter condition in coding an unordered multistate character (first dorsal rib long, extends full length of first costal; intermediate, in contact with axillary buttress; intermediate to short, extends less than halfway across the first costal). Coded that way, this character becomes partly dependent on character 119 of the present analysis (character 84 of Joyce 2007), which codes for the contacts of the axillary buttress (see above). Moreover, a survey of the taxa included in the analysis shows that the development of the axillary buttress is highly variable among turtles and that it cannot be used as a stable landmark to score the length of the first dorsal rib. Sterli et al. (2007, ch. 72 ) also coded the length of the first dorsal rib as a multistate character (extends to peripherals or nearly so and lies close to the tip of the axillary buttress; extends more than halfway across the first costal, but is far from the axillary buttress; extends less than halfway across the first costal). The intermediate state of Sterli et al. (2007) is only present in Notoemys laticentralis and is consequently uninformative in both their analysis and the present study. Neither the coding of Joyce (2007) nor that of Sterli et al. (2007) appears to be appropriate. The present coding is derived 
from Dryden (1988, ch. 37), Gaffney et al. (1991, ch. 35), Gaffney (1996, ch. 32), Brinkman \& Wu (1999, ch. 32) and Gaffney et al. (2007, ch. 87), and distinguishes taxa with a long first dorsal rib from taxa in which the rib is significantly reduced. Taxa with a well developed axillary buttress, which prevents the rib from extending the full length of the first costal, are scored state 0 because the first dorsal rib is always well developed in length in those taxa and it would certainly run the full length of the first costal if it was not for the buttress.

Character 154. Contact of dorsal ribs 9 and 10 with costals: 0 = present; 1 = absent (Joyce 2007, ch. 114).

Character 155. Dorsal rib 10: $0=$ long, spanning full length of costals and contacting peripherals distally; $1=$ short, not spanning farther distally than pelvis (Joyce 2007, ch. 115). In contrast to Joyce (2007; state 1), Santanachelys gaffneyi is scored as unknown because published description does not allow indisputable scoring of this character (Hirayama 1998).

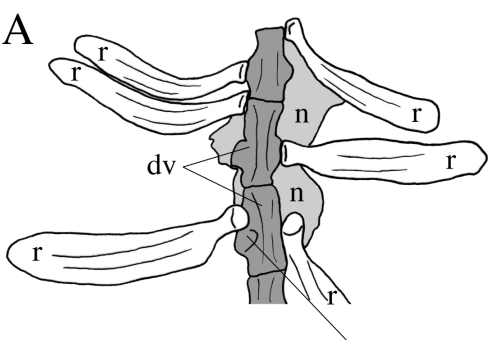

ch. $156(0)$

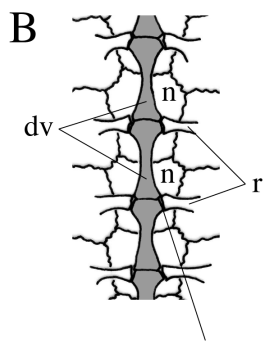

ch. $156(1)$
Figure 6 - Illustration of states for character 156 . Dorsal vertebrae and ribs of Odontochelys semitestacea (A) and Geoclemys hamiltonii (B). (A) redrawn from Li et al. (2008) and (B) redrawn from Joyce (2007). Abbreviations: $d v$, dorsal vertebra; $n$, neural plate; $r$, rib.

Character 156 (Fig. 6). Contact between dorsal vertebrae and dorsal ribs: $0=$ transverse processes of all dorsal vertebrae in the middle or anterior part of the vertebral centra; $1=$ dorsal ribs in contact with two successive vertebral centra (new character; derived from Rieppel \& Reisz 1999; Li et al. 2008; Joyce et al. 2009). All turtles, with the notable exception of Odontochelys semitestacea, are characterised by a peculiar relationship between the dorsal vertebral centra and ribs: each dorsal rib contacts the vertebrae on the boundary between two successive centra (Hoffstetter \& Gasc 1969; Rieppel \& Reisz 1999: 15; Joyce et al. 2009: 509). This apparently results from the forward migration by half a segment of neural arches in the dorsal region of the vertebral column (Rieppel \& Reisz 1999: 14; and references therein). In Odontochelys semitestacea (Li et al. 2008) and the majority of other reptiles (e.g., Romer 1956), the transverse process of each dorsal vertebra is located around the middle of the centrum. According to Joyce et al. (2009), the contact of dorsal ribs with two successive centra is only incipient in the Norian turtle Chinlechelys tenertesta (not included in the present analysis given the fragmentary nature of the material). According to Gaffney (1990: 119) this articulation between dorsal ribs and two successive centra "occurs in all turtles [known at that time] to some extent but usually the posterior thoracics tend to lose the articulation with the posterior ribs and maintain a complete articulation with their own rib anteriorly" (see also Hoffstetter \& Gasc, 1969). This is illustrated by Palaeochersis talampayensis in which dorsal ribs 6 through 9 articulate with their own centrum, whereas at least dorsal ribs 2 and 10, the others being lost, articulate with two successive centra (Sterli et al. 2007: 32 and fig. 8).

Character 157. Anterior articulation of the first dorsal centrum: 0 = faces at most slightly anteroventrally; 1 = faces strongly anteroventrally (Joyce 2007, ch. 116).

Character 158. Chevrons: $0=$ present on nearly all caudal vertebrae; 1 = absent, or only poorly developed, along the posterior caudal vertebrae (Joyce 2007, ch. 117).

Character 159. Tail club: 0 = present; 1 = absent (Joyce 2007, ch. 118).

Character 160. Caudal centra: $0=$ amphicoelous; $1=$ procoelous or opisthocoelous (Sterli 2008, ch. 130). The evolution of the caudal vertebral articulations of turtles is very complex and successive authors have tried to anatomise it in many ways (see Joyce, 2007: 47). For example, Joyce (2007, ch. 119) coded an unordered multistate character regrouping, according to him, the four basic patterns of caudal vertebral articulations found in turtles (all centra amphicoelous; all centra procoelous; all centra opisthocoelous; anterior centra procoelous, posterior centra opisthocoelous). Beyond the problems of unwarranted character state homology, the mere repartition of the different states suggests a high level of homoplasy resulting in a very complex evolutionary history (Joyce, 2007: 47). Moreover, characters related to caudal vertebral articulation are often difficult to score for fossil taxa, because caudal vertebrae are sparsely preserved and complete caudal column very rare. In this context, the simple coding proposed by Sterli (2008, ch. 130), although not entirely satisfactory, is preferred here pending further investigation.

Character 161. Morphology of the scapulocoracoid: 0 $=$ horizontal blade with a dorsal process, not triradiate; 1 $=$ triradiate in shape, with the development of an acromial process (Sterli et al. 2007, ch. 76; Sterli 2008, ch. 
131). The present study disagrees with Sterli et al. (2007) and Sterli (2008) in the scoring of Proganochelys quenstedti and Palaeochersis talampayensis. In both species the morphology of the scapulocoracoid is similar (see Sterli et al. 2007: 36) and there is an incipient acromial process, so that the pectoral girdle of these species is indeed triradiate in shape (state 1). Li et al. (2008) described the acromial process as missing in Odontochelys semitestacea, but the pectoral girdle of this species does not appear to be fundamentally different from that of Proganochelys quenstedti and a similarly developed acromial process appears to be present ( $\mathrm{Li}$ et al. 2008: fig. 3c). The acromial process of turtles is believed not to be homologous with the acromial process found in pareiasaurs (deBraga \& Rieppel 1997: 302), so the later are scored with state 0 in the present analysis.

Character 162. Cleithrum: $0=$ present; $1=$ absent (modified from Joyce 2007, ch. 120). Joyce (2007) coded an ordered multistate character for the absence/presence and contacts of the cleithrum in turtles (present, in contact with the carapace; present, contact with carapace absent; absent). According to the coding strategy followed herein and in order to allow the scoring of outgroups which do not have shells, the present analysis codes two separate binary characters for the cleithrum (characters 162 and 163). Here, I follow the proposal of Joyce et al. (2006) that the epiplastral processes of turtles are indeed cleithra. The arguments expressed by these authors are based on good evidence and it appears reasonable to assume they are accurate. However, these arguments are considered ambiguous by others (e.g., Rieppel 2008). Whether the so-called 'epiplastral process' of turtles indeed corresponds to the cleithrum of other amniotes or to a mere dorsal expansion of the epiplastron (i.e., of the clavicle) is not fundamental issue for the purpose of the present analysis. This question would be important however in the context of an analysis of the relationships of turtles within amniotes, notably because the cleithrum is undoubtedly lost in crown-group diapsids (Sauria) and because an inaccurate primary homology assessment might support false relationships for turtles.

Character 163. Osseous contact of cleithrum with carapace: 0 = present; 1 = absent (modified from Joyce 2007, ch. 120). See character 162 for a discussion. Li et al. (2008) scored Odontochelys semitestacea as having a contact of the cleithrum with the carapace, but this contact cannot exist in a form in which the carapace is almost non-existent. Consequently, Odontochelys semitestacea is scored inapplicable in the present analysis. Taxa that have lost the cleithrum are scored inapplicable for this character.
Character 164. Length of acromial process: $0=$ less than one half the length of scapular process; $1=$ more than one half the length of scapular process (Joyce 2007, ch. 121). Following Joyce \& Sterli (pers. comm., 2007), the scoring of Kayentachelys aprix has been changed from unknown to state 0 .

Character 165. Shape of acromial process: $0=$ triradiate in section, acromial ridge present; 1 = rod-like (Joyce 2007, ch. 122; Sterli et al. 2007, ch. 75).

Character 166. Glenoid neck on scapula: 0 = absent; 1 $=$ present (Joyce 2007, ch. 123).

Character 167. Coracoid foramen: 0 = present; $1=$ absent (Joyce 2007, ch. 124).

Character 168. Sutural articulation of pelvis to shell: 0 = absent; 1 = present (Joyce 2007, ch. 125). Following the publication of Joyce (2007), Sterli et al. (2007) confirmed that the pelvis of Palaeochersis talampayensis is sutured to the shell, so this species is scored state 1 in the present analysis. As suggested by Joyce (2007: 48), the condition in Proterochersis robusta remains ambiguous and this species is scored unknown for this character.

Character 169. Elongated iliac neck: 0 = absent; $1=$ present (Joyce 2007, ch. 126).

Character 170. Shape of the ilium articular site on the visceral surface of the carapace: $0=$ narrow and pointed posteriorly; 1 = oval (Joyce 2007, ch. 128).

Character 171. Posterior notch in acetabulum: $0=$ absent; 1 = present (Joyce 2007, ch. 129).

Character 172. Ischial contacts with plastron: $0=$ contact via a large central tubercle; 1 = contact via two separate ischial processes (Joyce 2007, ch. 130). The morphology of Odontochelys semitestacea regarding this character is unclear. Li et al. (2008) described a posterior ischial tubercle in this species, but they also stated that the ischium is located behind the plastron so that a contact between the ischium and plastron is absent. This morphology may be the result of post-mortem deformation ( $\mathrm{Li}$ et al. 2008: fig. 2a, b). Odontochelys semitestacea is scored as unknown in the present analysis. The ischial morphology in Palaeochersis talampayensis appears to be different from that of other turtles: the posteromedian part of the ischium is sutured to the xiphiplastron, but no central tubercle is present and the lateral ischial processes do not appear to contact the plastron (Sterli et al. 2007: 40). Consequently, this species is also scored as unknown in the present analysis.

Character 173. Hypoischium: $0=$ present; $1=$ absent (Joyce 2007, ch. 131). Odontochelys semitestacea, Proganochelys quenstedti and Palaeochersis talampayensis are the only turtles known to have hypoischia, i.e. single 
or paired postpelvic ossifications that articulate with the ischia anteriorly. The homology between these chelonian postpelvic ossifications and the structures termed hypoischia in some other reptiles, especially lizards, is not well established (Romer 1956; Gaffney 1990). Consequently, all of the outgroups are scored as unknown for the present character.

Character 174. Fibula: 0 = bowed away from tibia; 1 = straight, not bowed away from tibia (deBraga \& Rieppel 1997, ch. 145; Müller, J. 2004, ch. 149; Hill 2005, ch. 281). This character is included for outgroup comparison. The plesiomorphic condition among amniotes is a fibula that is bowed away from the tibia (deBraga \& Rieppel 1997: 311). In turtles, Araeoscelidia and Neodiapsida, the fibula is straight and not bowed away from the tibia.

Character 175. Phalangeal formula of the manus: $0=$ 2-3-4-4-3 or more; 1 = digits 2 to 5 with three phalanges or less (modified from Joyce 2007, ch. 132). Character 132 of Joyce (2007) originally coded the phalangeal formula as a binary character (most digits with three elongate phalanges; most digits with two short phalanges). In the context of the present analysis, which includes Odontochelys semitestacea and non-chelonian outgroups, this character is not sufficient. The plesiomorphic reptilian condition is a phalangeal formula of $2-3-4-5-3$, or exceptionally of 2-3-4-4-3 (Romer 1956). Odontochelys semitestacea is unique among turtles in having a manual phalangeal formula of 2-3-4-4-3 (Li et al. 2008). Within remaining turtles, the phalangeal formula of the manus varies greatly but generally does not exceed 2-3-3-3-3 and is even reduced to $2-2-2-2-1$ in the most extreme case of terrestrial adaptation (Romer 1956). In the present analysis, the phalangeal formula of the manus has been separated into two binary characters. Character 175 codes for the acquisition of the reduced phalangeal formula consisting of most digits having three phalanges or less, which unites all turtles with the exception of Odontochelys semitestacea. Character 176 (see below) concerns the acquisition of an even more reduced phalangeal formula of the manus where most digits have only two short phalanges, which is interpreted to be a terrestrial adaptation (Joyce \& Gauthier 2004).

Character 176. Reduced phalangeal formula of the manus, all digits with only one or two short phalanges: 0 = absent; 1 = present (modified from Joyce 2007, ch. 132). See character 175 for a discussion.

Character 177. Paddles: $0=$ absent; $1=$ present (modified from Joyce 2007, ch. 133). Joyce (2007) coded the development of paddles as an ordered multistate character (absent; short paddles; elongate paddles). However, Joyce (2007) was not explicit regarding the boundary between short and elongate paddles and this is a distinction that is not always easy to make for fossil taxa. For these reasons, only the absence/presence of paddles is considered here. Paddles are characterised by immobile digits bound together by connective tissue (Joyce 2007: 52). Hirayama (1998: 705) clearly stated that Santanachelys gaffneyi retains movable digits, as in freshwater turtles, so that paddles, strictly speaking, are absent in this species. This departs from Joyce (2007) who scored Santanachelys gaffneyi as possessing short paddles.

Character 178. Claw of the fifth digit of the pes: $0=$ present; 1 = absent (Joyce 2007, ch. 135).

\section{Cladistic analysis}

\section{Methods}

The data matrix (see Supplementary Material) of 178 binary characters scored for 93 taxa (including 64 fossil and 22 living species of turtles and 7 fossil outgroups) was assembled with MacClade 4.01 (Maddison \& Maddison 2001). The parsimony analyses were performed using PAUP 4.0b10 (Swofford 2002). Trees were rooted on the seven outgroups (see above). Characters were considered reversible and equallyweighted. Branches were set to collapse if their minimal length was zero. Due to computational limitations, nonstrategic heuristic searches with PAUP failed to find the shortest trees before the memory limit was hit. Consequently, the data matrix was analysed using the parsimony ratchet method (Nixon 1999). Originally designed for the analysis of large data set, this method uses a combination of tree bisection-reconnection (TBR) branch-swapping and an algorithm that alternatively reweights a randomly selected percentage of the character, allowing the search to 'jump' between 'tree islands' and consequently to explore tree space more thoroughly. Character weights are reset to their original value before TBR branch-swapping, so that reweighting does not affect the resulting topology. In the present analysis, the parsimony ratchet was implemented in PAUP 4.0b10 using PAUPRat (Sikes \& Lewis 2001) and set to perform 200 iterations and reweight $15 \%$ of characters. Nixon (1999) and Goloboff (1999) argue that the consensus of the combined results of a sufficient number of parsimony ratchet searches (i.e., between 10 and 20) should be identical to the consensus of all the shortest trees for a given matrix. In the present analysis, 20 independent parsimony ratchet searches were performed. Bremer support values (= decay indices) were calculated with PRAP 2.0b3 (Müller, K. 2004, 2007), which also uses the parsimony ratchet method (200 iterations, $15 \%$ reweighting, 20 independent searches). 
In addition to the aforementioned methodology, the present analysis also used the safe taxonomic reduction (Wilkinson 1995b) and strict reduced consensus (Wilkinson 1994, 1995a) methods. Safe taxonomic reduction (STR) is a simple method to identify those taxa which exclusion prior to the analysis does not alter the relationships among remaining taxa, but could dramatically reduce the number of equally parsimonious solutions and consequently reduce computing time (Wilkinson 2003). STR was implemented using TAXEQ3 (Wilkinson 2001). The strict reduced consensus (SRC) method identifies all strictly supported relationships for a given data set by overcoming the insensitivity of the strict reduced consensus method and the ambiguous interpretation of the Adams consensus tree (Wilkinson 2003; see below). SRC trees were computed using RadCon 1.1.6 (Thorley \& Page 2000).

\section{Interpretation of consensus trees}

Consensus trees are undoubtedly the most widespread mean of summarising cladistic information. The three more common consensus methods are: the strict consensus, the majority-rule consensus and the Adams consensus. Neither of these methods, as any other consensus method, is free from imperfection. The strict consensus is often deemed too strict, or insensitive, especially when taxa with a significant amount of missing data (e.g., fossils) are considered (e.g., Wilkinson 1994, 2003). Concerning the majority-rule consensus, it has been demonstrated that when ambiguity is rampant within a data matrix, especially because of missing data, biases in the method may drive the consensus tree toward the most ambiguous set of topologies (Sharkey \& Leathers 2001; Sumrall et al. 2001). Finally, the interpretation of the Adams consensus if often ambiguous because it does not represent common components/clades as strict and majority-rule consensus do, but common nestings (see Wilkinson 1994 and Kitching et al. 1998 for an introduction to the literature relating to consensus trees). Hence, groups may appear in an Adams consensus that are not present in all, if any, fundamental trees. Ambiguity also arises from the fact that unstable taxa are relocated at the lowest common node in the Adams consensus tree, but such taxa cannot be identified without doubt in the resulting tree.

A theoretical example with five taxa (A, B, C, D, and E) and two fundamental cladograms ( $T 1$ and $\mathrm{T} 2$ ) will illustrate the ambiguous interpretation of the Adams consensus tree (Fig. 7). In the strict consensus tree (Fig. 7C), only the clade formed by $D$ and $E$ is resolved in the ingroup, because this is the only component/clade shared by the two fundamental trees (Fig. 7A, B). The Adams

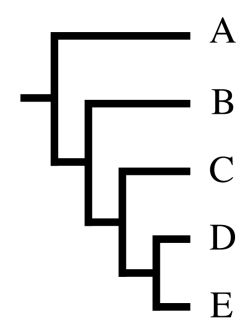

$\mathrm{T} 1$

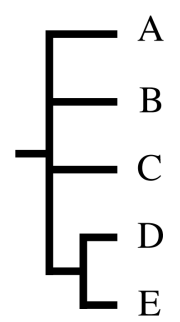

Strict

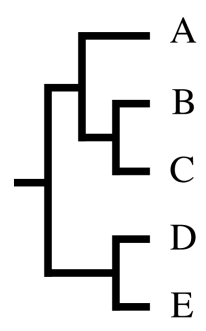

$\mathrm{T} 2$

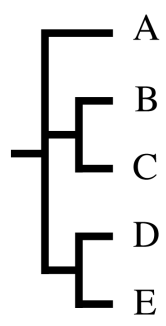

Adams

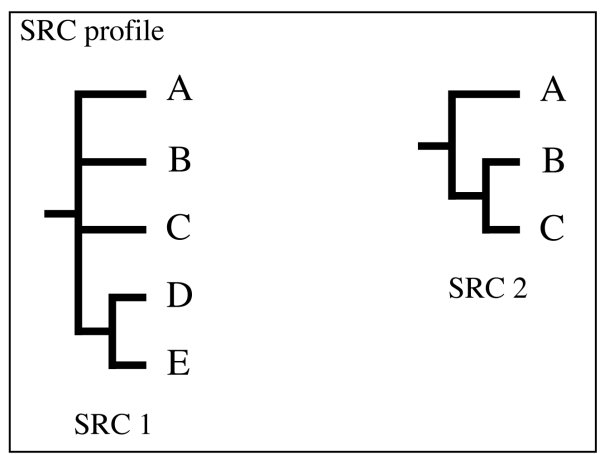

Figure 7 - Interpretation of consensus trees. Theoretical example with two fundamental cladogram ( $\mathrm{T} 1$ and $\mathrm{T} 2$ ) and corresponding strict consensus, Adams consensus and Strict Reduced Consensus (SRC) profile.

consensus shows a basal polytomy grouping A with two nestings formed by $B$ and $C$, and $D$ and $E$, respectively (Fig. 7D). If $D$ and $E$ are always more closely related to each other than either is to any other taxon (i.e., they form a clade in all fundamental trees), this is not the case of $B$ and $C$. In $T 1, C$ is more closely related to $D$ and $E$ than to $B$. In the case of $B$ and $C$, the Adams consensus tree should be interpreted as follows: in all fundamental trees, $B$ and $C$ share a node in common that is distinct from the node including $A, B, C, D$ and $E$.

As a complement to the aforementioned consensus, Wilkinson (1994, 1995a) proposed the strict reduced consensus (SRC) method. The SRC profile is composed of a variable number of trees corresponding to the strict consensus tree from which one or several taxa are pruned a posteriori in order to reveal (graphically) all unambiguously supported n-taxon statements. SRC trees 
are very useful as an aid to interpret Adams consensus trees. In the theoretical example detailed above, the SRC profile contains two SRC trees. The first (Fig. 7E) is also the strict consensus tree (i.e., no taxon is pruned and shown n-taxon statements are indeed components/ clades). In the second SRC tree, taxa D and $E$ are pruned to reveal a 3-taxon statement between A, B and C that is supported by all fundamental trees (Fig. 7F). The interpretation of SRC 2 is not ambiguous: in all fundamental trees, $B$ and $C$ are more closely relate to each other than either is to $A$, but $D$ and $E$ have unstable relationships with respect to these taxa. This does not imply that B and C form a clade in any fundamental tree. Hence, the SRC method provides cladistic information that was not readily available with either the strict consensus or the Adams consensus (i.e., the respective relationships between $A, B$ and $C$ ) and offers a mean of identifying taxa with unstable relationships and the extent of their instability (i.e., with respect to which specific taxa they are unstable).

\section{Results}

The safe taxonomic reduction method (Wilkinson 1995b) revealed that Australochelys africanus, Ninjemys oweni, Chengyuchelys zigongensis and Apalone ferox could be discarded prior to the analysis without altering the relationships among remaining taxa. Furthermore, the STR analysis indicated that these four species would arise from the same node as their index taxon (i.e., Palaeochersis talampayensis, Meiolania platyceps, Chengyuchelys baenoides and Lissemys punctata, respectively) in any most parsimonious tree (MPT), allowing their placement in the cladogram a posteriori. As a result of the exclusion of the four aforementioned species the following eight characters are parsimony uninformative: 4, 24, 32, 36, 69, 115, 116, and 152. These would be synapomorphies of Meiolania platyceps + Ninjemys oweni (chs 4, 24, 32, and 69), Chengyuchelys baenoides $+C$. zigongensis (ch. 116), and Lissemys punctata + Apalone ferox (chs 36, 115, and 152), which allows to precise the placement of the discarded taxa a posteriori (see data matrix in Supplementary Material). Hence, $N$. oweni, C. zigongensis and A. ferox not only arise from the same node as their respective index taxon, but also form a sister group with it (Fig. 8).

The parsimony analysis of the data matrix (excluding the four aforementioned taxa) with the parsimony ratchet method (see above) resulted in 403 distinct trees of 439 steps. These trees have a Consistency Index (CI) of 0.4055 (after exclusion of uninformative characters) and a Retention Index (RI) of 0.8075 . The low $\mathrm{Cl}$ accounts for the high level of homoplasy within the data matrix, but the rather high $\mathrm{RI}$ indicates that a significant part of this similarity can be interpreted as synapomorphy (i.e., it is phylogenetically informative). These indices are relatively similar to those obtained by Joyce (2007) for his three analyses, the main divergence rests in the slightly lower $\mathrm{Cl}$ of the present study (0.4055 against $0.46-0.47)$. This can be at least partly explained by the increased number of characters and taxa in the present study (Kitching et al. 1998).

The strict consensus tree (766 steps; $\mathrm{Cl}=0.2243 ; \mathrm{RI}=$ 0.5664 ) is poorly resolved (Fig. 8A). Overall, Bremer support values are relatively low (i.e., 1 or 2 ), with the exception of panpleurodires and kinosternids. The Adams consensus tree is 472 steps long and has a $\mathrm{Cl}$ of 0.3664 and a RI of 0.7832 (Fig. 8B). In order to facilitate the interpretation of the Adams consensus tree and help identify taxa with unstable relationships (see above), strict reduced consensus (SRC) were computed for the 403 shortest trees found with the parsimony ratchet method. The SRC profile contained 24 SRC trees in which ambiguous taxa are alternatively pruned from the strict consensus tree in order to reveal unambiguous relationships (Fig. 9). These results are discussed below. A $50 \%$ majority-rule consensus tree is also provided for comparison in the Supplementary Material.

\section{Discussion}

If comparison needs to be made, then the present analysis is more comparable with the first analysis of Joyce (2007), in which wildcard taxa were included and all characters left unordered. The results of the first analysis of Joyce (2007) were rightly deemed as counterintuitive by their author, especially because of the position of panpleurodires high up within Cryptodira. In order to obtain a more intuitive topology, Joyce (2007) relied on the exclusion of wildcard taxa (Portlandemys mcdowelli, Sandownia harrisi, and Mongolemys elegans) and on the ordering of 15 multistate characters. The present analysis, being largely based on that of Joyce (2007), unsurprisingly confirms most results of the latter study, especially the existence of an extensive stem to the turtle crown group (Testudines). Indeed, the topology obtained herein (Fig. 8B) greatly recalls that obtained by Joyce (2007), following his third protocol. Consequently, the changes proposed in this paper, and especially the coding strategy, may appear useless or at least without significant impact on the resulting topology. That would be forgetting that the present study recovers such a topology without the need to exclude wildcard taxa, and actually includes 19 additional species, several of which would be deemed as wildcards by most; without also the need to constrain the analysis by ordering multistate 
A

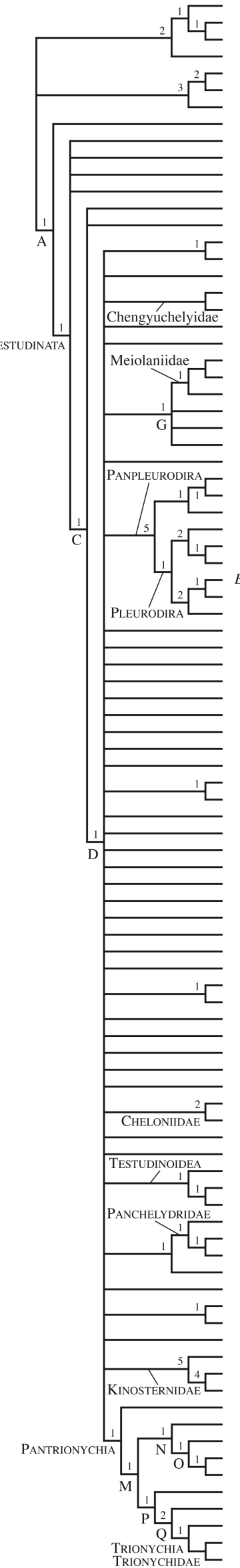

Anthodon serrarius Araeoscelidia

Owenetta kitchingorum

Simosaurus gaillardoti

Protorosaurus speneri

Pephyrosaurus bridensis

Odontochelys semi

Proterochersis robu

Proterochersis robusta

Proganochelys quenstedti

Palaeochersis talampayensi

Australochelys africanus

Kayentachelys aprix

Indochelys spatulata

Heckerochelys romani

Condorchelys antiqua

Sichuanchelys chowi

Chengyuchelys baenoides

Chengyuchelys zigongensis

'Chengyuchelys' dashanpuensis

Eileanchelys waldmani

Meiolania platyceps

Ninjemys oweni

Niolamia argentina

Mongolochelys efremovi

Otwayemys cunicularius

Naomichelys speciosa

Kallokibotion bajazidi

Platychelys oberndorferi

Caribemys oxfordiensis

Notoemys laticentralis

Elseya dentata

Chelodina oblonga

Phrynops geoffroanus

Erymnochelys madagascarien

Pelomedusa subrufa

Podocnemis expansa

Arundelemys dardeni

Pleurosternon bullocki

Glyptops plicatulus

Dinochelys whitei

Dorsetochelys delairi

Neurankylus eximius

Trinitichelys hiatti

Plesiobaena antiqua

Boremys pulchra

Baena arenosa

Chisternon undatum

Plesiochelys solodurensis

Portlandemys medowelli

Solnhofia parsonsi

Santanachelys gaffneyi

'Thalassemys moseri'

Xinjiangchelys qiguensis

Siamochelys peninsularis

Xinjiangchelys tianshanensi Xinjiangchelys latimarginalis Annemys levensis

Sinemys lens

Ordosemys leios

Dracochelys bich

Dracochelys hicuspis

Hangaiemys hoburensi

Judithemys sukhanovi
Toxochelys latiremis

Mesodermochelys undulatu

Caretta caretta

Chelonia mydas

Dermochelys coriacea

Mongolemys elegans

Chrysemys picta

Chrysemys picta

Geoclemys hamiltonii

Geoclemys hamiltonii

Protochelydra zangerli

Macrochelys temmincki

Chelydra serpentina

Platysternon megacephalum

Emarginachelys cretacea

Baptemys wyomingensis

Dermatemys mawii

Hoplochelys crassa

Staurotypus triporcatus

Staurotypus triporcatus

Sternotherus odoratus
Kinosternon flavescens

Adocus beatus

Basilemys variolos

Sandownia harrisi

Zangerlia neimongolensis

Chubutemys copelloi

Peltochelys duchastelli

Pseudanosteira pulchra

Carettochelys insculpto

Apalone ferox

Lissemys punctata
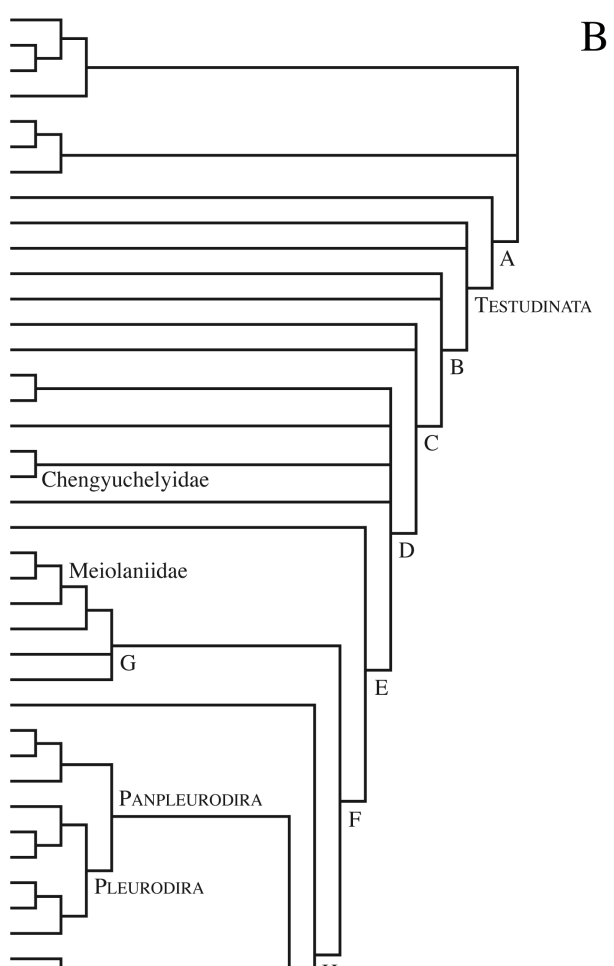

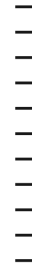

弱

Pleurosternida

$\mathrm{H}$

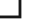

PARACRYPTODIRA

D. Baenidae
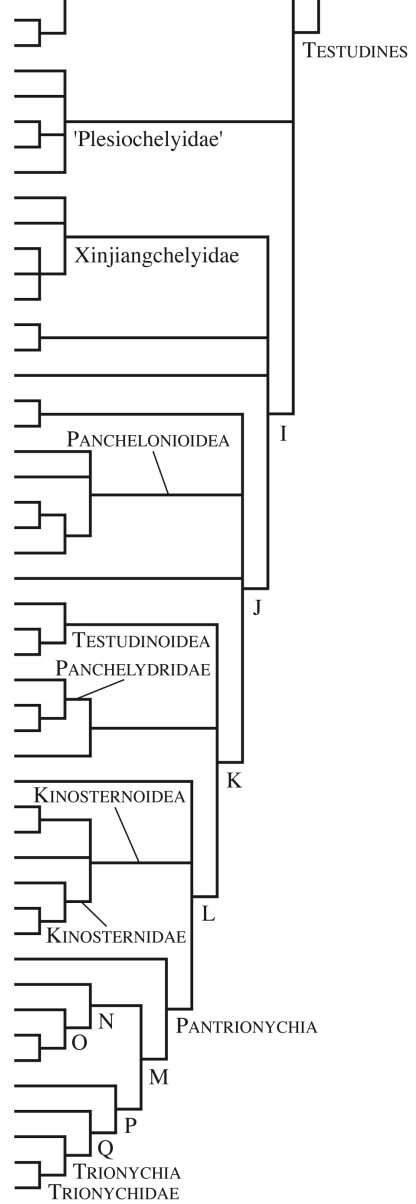
Figure 8 (previous page) - Phylogenetic interrelationships of turtles resulting from the analysis of the complete date set. (A) strict consensus (766 steps; $\mathrm{Cl}=0.2243 ; \mathrm{RI}=0.5664$ ) of 403 fundamental trees; (B) Adams consensus ( $472 \mathrm{steps} ; \mathrm{Cl}=0.3664 ; \mathrm{RI}=0.7832$ ) of 403 fundamental trees. Names in small capitals are defined phylogenetically. Capital letters label unnamed nodes. Numbers on the strict consensus tree correspond to Bremer support values.

characters; and finally by lifting another level of constraint by recoding multistate characters (within which the homology between character states cannot be tested by congruence) into binary variables. Retrospectively, the fact that similar topologies are obtained at the end suggests that most untested assumptions inherent to Joyce's (2007) third protocol and coding strategy were actually justifiable (as shown in some aspects by the following discussion), although in different context (additional taxa and/or characters) this may not hold true.

Unless otherwise stated, the following discussion is based on the topology of the Adams consensus tree (Fig. $8 \mathrm{~B})$. The complementary results provided by the SRC analysis are discussed where appropriate. SRC trees are represented as subtrees in Figure 9 and a list of pruned taxa is provided in the Supplementary Material for each SRC tree. A complete list of unambiguous apomorphies and DELTRAN optimisations is provided as Supplementary Material, but the synapomorphies of the major clades are discussed below. Unnamed nodes discussed in the text have been labelled with capital letters in order to avoid lengthy descriptions. This labelling is reported on relevant illustrations.

\section{Node A}

This clade (Fig. 8A) unites Odontochelys semitestacea and Testudinata (sensu Joyce et al. 2004). It is supported by the following unambiguous synapomorphies: postfrontal absent (character/state: 14/1); transverse process of cervical vertebrae in the middle of the centrum (ch. 142/0); and scapulocoracoid triradiate in shape (161/1). The present analysis supports the results of Li et al. (2008) regarding the phylogenetic position of 0 . semitestacea.

\section{Testudinata Klein, 1760 (sensu Joyce et al. 2004)}

The monophyly of Testudinata, the apomorphy-based clade uniting all tetrapods possessing a turtle shell (see character 86, above), is supported by the following unambiguous synapomorphies: teeth on lower and upper jaws absent (ch. 39/1); bony turtle shell present (ch. 86/1); dorsal ribs in contact with two successive vertebral centra (ch. 156/1); and digits 2 to 5 with three phalanges or less (ch. 175/1). This node consists of a polytomy between Proganochelys quenstedti, Proterochersis robusta and a group uniting all remaining turtles (node B; Fig. 8B). This is an artificial polytomy resulting from the Adams consensus method. The second SRC tree indicates that Proterochersis robusta is unstable with respect to the relationships of Proganochelys quenstedti and Palaeochersis talampayensis (Fig. 9). When Proterochersis robusta is pruned a posteriori, the unambiguously supported relationships of the other two taxa can be represented graphically: Proganochelys quenstedti is more basal than Palaeochersis talampayensis, which is in turn more basal than all remaining turtles. Hence, in the present analysis the phylogenetic position of Proterochersis robusta is partly uncertain, yet it can be concluded that this species is more derived than Odontochelys semitestacea but more basal than Kayentachelys aprix. The unstable behaviour of Proterochersis robusta relative to other basal turtles is probably the consequence of the presence of two pairs of mesoplastra (ch. 121/1) in Odontochelys semitestacea, suggesting that this may be the plesiomorphic condition for turtles (see character 121, above). However, other features suggest that Proterochersis robusta may be more derived than Proganochelys quenstedti and Palaeochersis talampayensis, which only have one pair of mesoplastra, these features include: the absence of anterior plastral tuberosities (ch. 132/1) and the presence of an elongated iliac neck (ch. 169/1; see node C, below).

\section{Node $B$}

This node consists of a polytomy between Palaeochersis talampayensis, Australochelys africanus and a node uniting all remaining turtles (i.e., node C; Fig. 8B). Node $B$ is unambiguously supported by the following synapomorphies: jugal-squamosal contact absent (ch. 20/1); vomerine and palatine teeth absent (ch. 45/1); cavum tympani present (ch. 49/1); incisura columellae auris present (ch. 52/1); basipterygoid articulation fused (ch. 58/1); and processus paroccipitalis of opisthotic tightly sutured to squamosal and quadrate (ch. 71/1).

\section{Node C}

This clade consists of a polytomy between Kayentachelys aprix, Indochelys spatulata and a node uniting all remaining turtles (i.e., node D; Fig. 8A, B). In the context of the present analysis, the exact relationships between Kayentachelys aprix and Indochelys spatulata are not resolved ('true' polytomy). This result is similar to that obtained by Sterli (2008). Hence, the present work and the latter study both support the conclusions of Datta et 
SRC 2

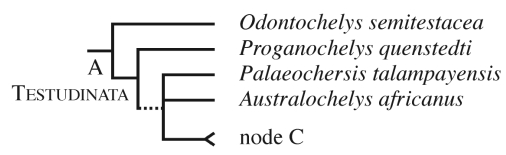

SRC 3

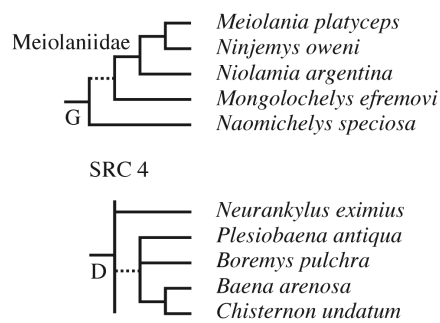

SRC 5

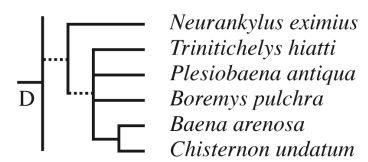

SRC 13

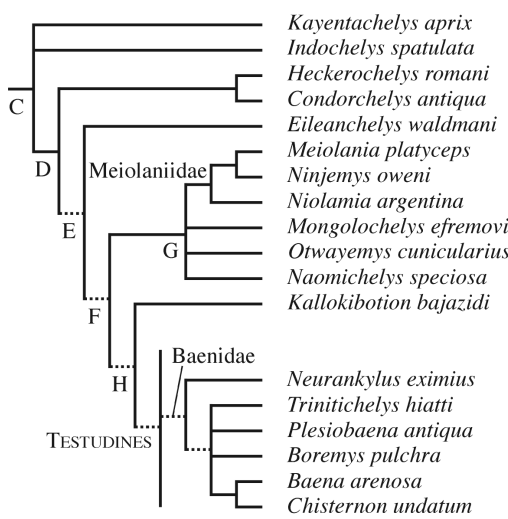

SRC 17

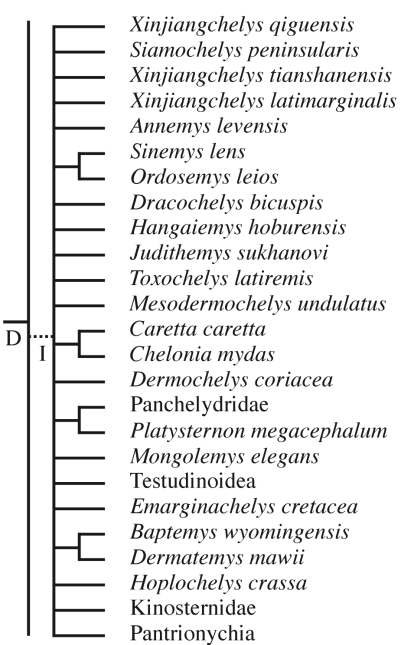

SRC 19

D.... Hangaiemys hoburensi Judithemys sukhanovi

SRC 20

1 - Sinemys lens

D.... Ordosemys leios
SRC 6

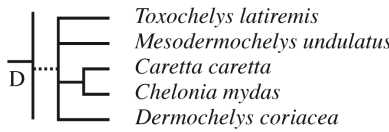

SRC 7

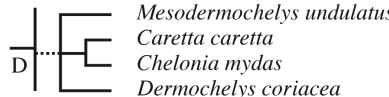

SRC 8

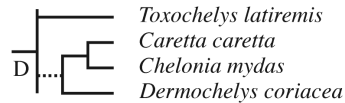

SRC 9

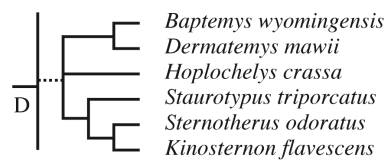

SRC 14

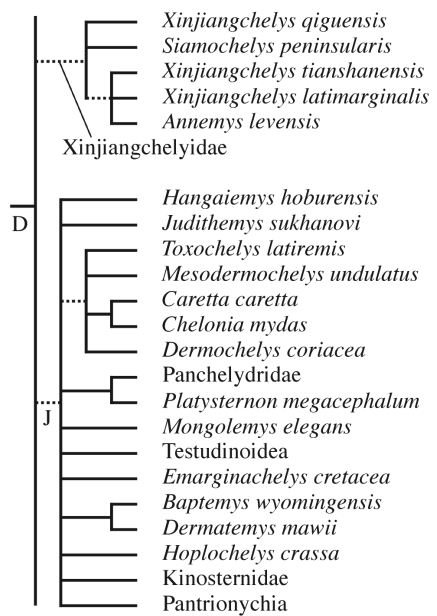

SRC 18

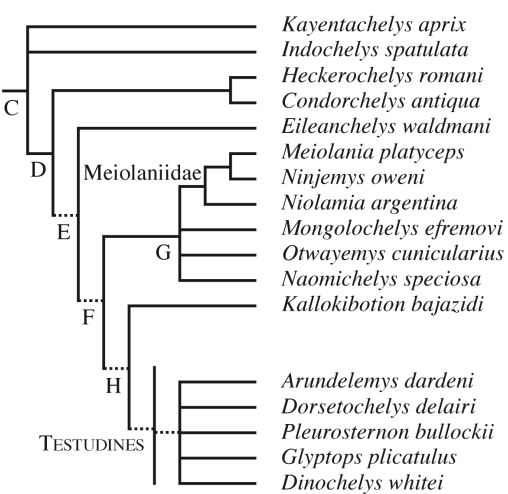

SRC 10

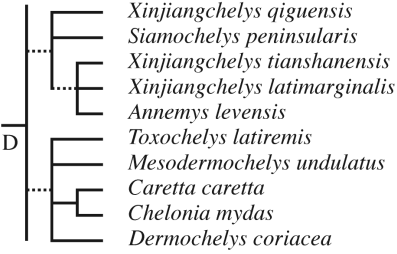

SRC 11

$$
\begin{array}{l|l}
\mathrm{D} \mid . .-\left[\begin{array}{l}
\text { Arundelemys dardeni } \\
\text { Pleurosternon bullockii } \\
\text { Glyptops plicatulus }
\end{array}\right. \\
\text { Dinochelys whitei }
\end{array}
$$

SRC 12

$\mathrm{D} . . .5$ Arundelemys dardeni
$\mathrm{D}$

RC 15

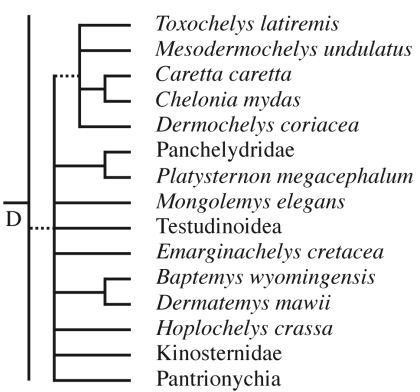

SRC 16

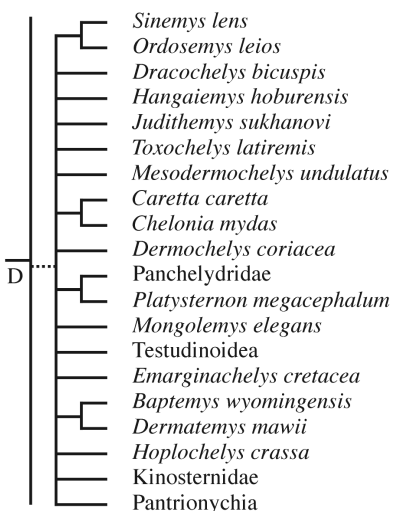

SRC 24

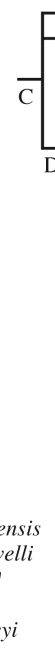

Kayentachelys aprix

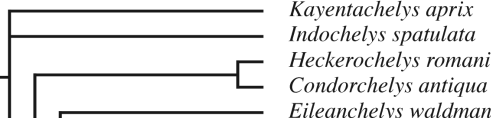

$\longrightarrow$ Eileanchelys waldmani

D Meiolaniidae - - Meiolania platyceps

Meiolaniidae - Ninjemys oweni

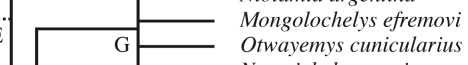

G $\square$ Otwayemys Naomichelys speciosa Kallokibotion bajazidi

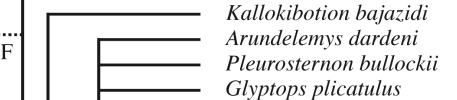

\begin{tabular}{|l|l}
\hline & Pleurosternon bullock \\
\hline & Glyptops plicatulus
\end{tabular}

Dorsetochelys delairi

Dinochelys whitei

$\int \sqrt{\square} \begin{aligned} & \text { Dinochelys whitei } \\ & \text { Neurankylus eximius }\end{aligned}$

$\int \sqrt{ } \begin{aligned} & \text { Dinochelys whitei } \\ & \text { Neurankylus eximiu }\end{aligned}$

1... 'Thalassemys moseri'

D -... $\begin{aligned} & \text { Solnhofia parsonsi } \\ & \text { Santanachelys gaffneyi }\end{aligned}$

SRC 22

5 Plesiochelys solodurensi

\begin{tabular}{c|l}
$\mathrm{D} \mid \mathrm{E}-\begin{array}{l}\text { Plesiochelys solodurensis } \\
\text { Portlandemys mcdowelli } \\
\text { Thalassemys moseri' }\end{array}$ \\
$\begin{array}{l}\text { Solnhofia parsonsi } \\
\text { Santanachelys gaffneyi }\end{array}$
\end{tabular} Plesiobaena antiqua

Boremys pulchra

- Baena arenosa

- Chisternon undatum

- Portlandemys mcdowelli

- 'Thalassemys moseri'

Solnhofia parsonsi

Solnhofia parsonsi 
Figure 9 (previous page) - Strict reduced consensus (SRC) profile of 403 fundamental trees (see text). SRC 1 corresponds to the strict consensus tree presented in figure 8A and is not represented here. SRC trees 2 to 24 are represented as subtrees, showing only the strictly supported n-taxon statements. A list of pruned taxa in each SRC tree can be found in the Supplementary Material. Dashed branches correspond to the gain of resolution with respect to the strict consensus tree (Fig. 8A).

al. (2000) who suggested a close relationships between these two Early Jurassic species. Clade $C$ is supported by numerous unambiguous synapomorphies: dorsal exposure of nasals greatly reduced relative to that of frontals (ch. 3/1); lacrimal absent (ch. 11/1); supratemporal absent (ch. 30/1); internarial process of premaxilla absent (ch. 31/1); anteroventral edge of the orbit formed mostly by maxilla (ch. 40/1); vomer single (ch. 42/1); central constriction of the middle ear by quadrate present (ch. 48/1); antrum postoticum present (ch. 51/1); supramarginals absent (ch. 105/1); anterior plastral tuberosities absent (ch. 132/1); osseous contact of cleithrum with carapace absent (ch. 163/1); coracoid foramen absent (ch. 167/1); elongated iliac neck present (ch. 169/1); and hypoischium absent (ch. 173/1).

\section{Node D}

This node consists of a broad polytomy grouping chengyuchelyids (see below), Sichuanchelys chowi, 'Chengyuchelys' dashanpuensis, Heckerochelys romani + Condorchelys antiqua and a node uniting all remaining turtles (i.e., node E; Fig. 8B). Node D is supported by the following unambiguous synapomorphies: pterygoid teeth absent (ch. 57/1); and anterior entoplastral process absent (ch. 113/1). SRC trees 5, 11, 12, 13, 18 and 24 reveals that chengyuchelyids, Sichuanchelys chowi and 'Chengyuchelys' dashanpuensis have highly unstable relationships (Fig. 9). Pruning of all or part of the latter species, sometimes with other taxa, allows the representation of strictly supported relationships among stem turtles and paracryptodires. The best illustration of this instability is SRC 13 (Fig. 9): when Chengyuchelys baenoides, 'Chengyuchelys' dashanpuensis and Sichuanchelys chowi are pruned, relationships of all taxa between Kayentachelys aprix and the turtle crown group (Testudines) are resolved, as well as relationships among Baenidae. Hence, the phylogenetic position of these three Chinese Middle Jurassic taxa is not resolved by the present data set, but their instability especially with respect to stem turtle relationships suggests that they may well belong to the phylogenetic stem of Testudines. This would be in contradiction with Danilov \& Parham (2008) who suggested that Chengyuchelys and Xinjiangchelys latimarginalis form a clade that is the sister group of Cryptodira. SRC trees 13, 18 and 24 reveal that the relationships of Heckerochelys romani and Condorchelys antiqua are unambiguously resolved by the present data set: these two species are more derived than Kayentachelys aprix, but more basal than Eileanchelys waldmani (Fig. 9).

The present data set supports the hypothesis of an extended stem to the turtle crown-group (Testudines) proposed by Joyce (2007), and especially the basal position of Kayentachelys aprix, meiolaniids, Mongolochelys efremovi and Kallokibotion bajazidi. In agreement with Sterli (2008) and Anquetin et al. (2009), the three recently described Middle Jurassic species $(H$. romani, $C$. antiqua and $E$. waldmani) fit well with this hypothesis and are phylogenetically placed between $K$. aprix and the group containing Meiolania platyceps and Mongolochelys efremovi. This is somewhat in contradiction with Gaffney \& Jenkins (2010) who reiterated their claim that $K$. aprix was a basal pancryptodire rather than a stem turtle. These authors suggested that $H$. romani, C. antiqua and $E$. waldmani might as well be early pancryptodires. However, evidence that the latter species are more basal than the panpleurodire-pancryptodire dichotomy goes beyond the list of characters used in the present analysis and several other features strongly indicate that they are stem turtles. It would be too long to discuss them here, so they will be detailed elsewhere.

\section{Heckerochelys romani + Condorchelys antiqua}

This clade (Fig. 8A) is unambiguously supported only by the retention of a central plastral fontanelle in adult individuals (ch. 111/1). Because of the concerns expressed about this feature (see character 111, above), this grouping should be considered with care until further evidence is available (e.g., additional material of Condorchelys antiqua). If Condorchelys antiqua is scored as unknown for character 111, then this clade is no longer supported by the analysis of the complete data set (parsimony ratchet; 200 iterations; 15\% reweighting; 20 replicates; branches set to collapse if minimum length is zero).

\section{Chengyuchelyidae Ye, 1990}

The clade formed by Chengyuchelys baenoides and $C$. zigongensis is reconstructed a posteriori based on STR results (see Results, above) and would be supported at least by the following synapomorphy: dagger-shaped entoplastron present (ch. 116/1). 


\section{Node $E$}

This node consists of a sister group relationship between Eileanchelys waldmani and a node uniting all remaining turtles (i.e., node F; Fig. 8B). It is supported by a single unambiguous synapomorphy: lateral contact of suprapygal 1 with peripherals present (ch. 95/1). This synapomorphy may not withstand further investigation: state 1 is only known in Eileanchelys waldmani, Naomichelys speciosa, Kallokibotion bajazidi, Plesiochelys solodurensis and Xinjiangchelys latimarginalis, whereas many early turtles (including meiolaniids, Mongolochelys efremovi, Portlandemys mcdowelli, Solnhofia parsonsi, and Santanachelys gaffneyi) are scored as unknown for this character (see Supplementary Material). This illustrates the need of further work on these turtles.

\section{Node F}

This node unites meiolaniids plus related species (see node $G$, below) and a node consisting of all remaining turtles (i.e., node $\mathrm{H}$; Fig. 8B). Node $\mathrm{F}$ is unambiguously supported by the following synapomorphies: vomer with a ventral median septum that separates the meatus choanae (ch. 43/1); posterior process of the pterygoid that floors the cavum acustico-jugulare present (ch. 60/1); and foramen jugulare posterius defined by bone (ch. 78/1)

\section{Node G}

This clade (Fig. 8A), which groups meiolaniids, Mongolochelys efremovi, Otwayemys cunicularius and Naomichelys speciosa, is supported by the following unambiguous synapomorphies: vertebral 5 does not overlap onto peripherals and pygal (ch. 109/1); central plastral fontanelle retained in adult individuals (ch. 111/1); and central cervical articulations formed, cervical vertebrae procoelous or opisthocoelous (ch. 145/1). Hirayama et al. (2000) were the first to propose a sister group relationships between meiolaniids and Mongolochelys efremovi, followed by Joyce (2007) and subsequent analyses based on the latter study (e.g., Danilov \& Parham 2008; Sterli 2008). Hirayama et al. (2000) also proposed that Otwayemys cunicularius was closely related to meiolaniids and Mongolochelys efremovi. Gaffney et al. (1998) indeed described Otwayemys cunicularius as closely related to meiolaniids, but Gaffney et al. (2007) did not find Mongolochelys efremovi to be closely related to meiolaniids. The present analysis agrees with Hirayama et al. (2000) in finding a close relationship between Otwayemys cunicularius and both meiolaniids and Mongolochelys efremovi. The relationships of Naomichelys speciosa have never been properly investigated in a phylogenetic context. This species is thought to be closely related to Helochelydra, which is traditionally interpreted as a pancryptodire genus (e.g., Hirayama et al. 2000; Milner 2004). Although it is not clearly stated, it seems that Hirayama et al. (2000) at least partly used FMNH PR273, an undescribed complete individual of Naomichelys speciosa, to score Helochelydra (= Treutosternon in Hirayama et al. 2000). These authors found Helochelydra to be the sister group of Kallokibotion bajazidi. The present analysis is the first to score Naomichelys speciosa as a separate terminal. The third SRC tree indicates that Otwayemys cunicularius has ambiguous relationships relative to Naomichelys speciosa and Mongolochelys efremovi (Fig. 9). The data set strictly supports Naomichelys speciosa to be more basal than Mongolochelys efremovi, which is congruent with the stratigraphic record, at least for the concerned specimens (see Supplementary Material).

\section{Meiolaniidae + Mongolochelys efremovi}

The node uniting meiolaniids and Mongolochelys efremovi is unambiguously supported by the following synapomorphies: squamosal-supraoccipital contact present (ch. 25/1); crista supraoccipitalis protruding posterior to foramen magnum (ch. 68/1); and ligamentous connexion between carapace and plastron (ch. 110/1). SRC tree 3 (Fig. 9) indicates that M. efremovi is more closely related to meiolanids than $N$. speciosa.

\section{Meiolaniidae Lydekker, 1889}

Meiolaniids are unambiguously supported by the following synapomorphies: meiolaniid 'horns' present (ch. 26/1); and intrapterygoid slit present (ch. 67/1). Within meiolaniids, the clade formed by Meiolania platyceps and Ninjemys oweni is reconstructed a posteriori based on STR results (see Results, above) and would be supported at least by the following synapomorphy: presence of an internarial process dividing the apertura narium externa formed by the nasal and premaxilla (ch. 32/1). The presence of a nasomaxillary sinus (ch. 4/1), of a quadratojugal-squamosal contact below the cavum tympani (ch. 24/1), and of a large supraoccipital exposure on the skull roof (ch. 69/1) are scored as unknown for Niolamia argentina and consequently may either be, depending on optimisation, synapomorphies of meiolaniids (ACCTRAN) or of the clade Meiolania platyceps + Ninjemys oweni (DELTRAN).

\section{Node $\mathrm{H}$}

This node consists of a sister group relationship between Kallokibotion bajazidi and Testudines (i.e., crowngroup turtles). Node $\mathrm{H}$ is supported by the following unambiguous synapomorphies: epipterygoid laminar (ch. 
56/1); pterygoid-basioccipital contact present (ch. 61/1); axillary buttress contacts peripherals and first costal (ch. 119/1); and inguinal buttress contacts peripherals and costals (ch. 123/1).

\section{Testudines Batsch, 1788 (sensu Joyce et al. 2004)}

This node consists of a polytomy between four groups (Fig. 8B): Panpleurodira, Paracryptodira, 'Plesiochelyidae' and node I (see below). Testudines (i.e., crown-group turtles) are unambiguously supported by the following synapomorphies: articulation of nuchal with neural spine of eighth cervical vertebra absent (ch. 89/1); contact between first suprapygal and peripherals absent (ch. 95/0); and glenoid neck on scapula present (ch. 166/1). The present data set is unable to resolve the relationships between the major groups of Testudines. This is similar to the results obtained by the three analyses of Joyce (2007), despite his 'preferred phylogenetic hypothesis' displays resolved relationships corresponding to the commonly accepted pattern.

This lack of resolution of phylogenetic analyses while most authors agree on the general pattern of relationships (i.e., panpleurodires as sister-group to pancryptodires, themselves splitting into paracryptodires and eucryptodires) is apparently the consequence of two phenomena. Firstly, taxon sampling is not homogenous. Whereas paracryptodires and eucryptodires are represented by numerous Mesozoic forms, panpleurodires are only represented by three relatively poorly known basal species from the Late Jurassic (Platychelys oberndorferi, Caribemys oxfordiensis and Notoemys laticentralis) and six derived extant species (see Supplementary Material). The morphological gap between these extant pleurodires and their basal relatives and with potential sister groups of panpleurodires is very important (see discussion in Joyce 2007). Secondly, several character complexes that were constructed by Gaffney (1975a), and developed since by E.S. Gaffney and co-workers notably, to support the dichotomy between panpleurodires and pancryptodires and the basal relationships within the latter have been recently questioned. This is illustrated by the recent debate between Sterli \& Joyce (2007) and Gaffney \& Jenkins (2010) about Kayentachelys aprix and whether it should be interpreted as a stem turtle or an early pancryptodire. This questioning about Gaffney's (1975a) character complexes have led to new definition of certain characters and weaker support for basal relationships among Testudines. It is now apparent that, in order to resolve these relationships, early crown-group turtles from the late Middle Jurassic and Late Jurassic are in need of renewed attention.
In the present data set, the clades Pancryptodira, Paracryptodira and Eucryptodira are not supported unambiguously. As a matter of fact, interrelationships between Panpleurodira, some paracryptodires and some eucryptodires are uncertain. The $50 \%$ majority-rule consensus tree illustrates this: in 56\% of the 403 fundamental trees, baenids are more closely related to panpleurodires than to pleurosternids (see Supplementary Material). Yet, the SRC profile provides some information about the relationships among basal Testudines. Firstly, SRC tree 13 (Fig. 9) shows that relationships from Kayentachelys aprix to Testudines are resolved by the present data set and that only Sichuanchelys chowi, 'Chengyuchelys' dashanpuensis and chengyuchelyids have ambiguous relationships among these taxa. In other words, the poor resolution of basal relationships within Testudines does not contradict their phylogenetic position as more derived than Kayentachelys aprix, Meiolania platyceps, Mongolochelys efremovi and Kallokibotion bajazidi. Secondly, SRC tree 24 (Fig. 9) reveals that pleurosternids and baenids are more closely related to each other than either is to 'plesiochelyids', but panpleurodires and all remaining eucryptodires are unstable with respect to the aforementioned taxa.

Although excluding taxa a priori is not the best option, the six extant pleurodires have been discarded from the matrix and a second analysis ran following the parameters outlined above. In this second analysis, panpleurodires were only represented by Platychelys oberndorferi, Caribemys oxfordiensis and Notoemys laticentralis. The search with the parsimony ratchet resulted in 641 distinct trees of 387 steps $(\mathrm{Cl}=0.4109 ; \mathrm{RI}$ $=0.8056)$. The strict (591 steps; $\mathrm{Cl}=0.2690 ; \mathrm{RI}=0.6317$ ) and Adams (410 steps; $\mathrm{Cl}=0.3878 ; \mathrm{RI}=0.7860$ ) consensus trees are presented in Figure 10. The topology of the Adams consensus is more in agreement with the traditional interpretation of basal relationships among Testudines (Fig. 10B). However, the SRC profile (not shown here) indicates that the relationships of panpleurodires are still unstable with respect to paracryptodires and eucryptodires. This suggests that a significant effort must be made in future analyses to include more Mesozoic panpleurodires and to investigate more deeply their relationships with other crown-group turtles, although this would be partly dependant on the discovery of new material.

\section{Panpleurodira Joyce et al., 2004}

Panpleurodires (Fig. 8A, B) are supported by numerous synapomorphies: loss of the posterior process of the pterygoid that floors the cavum acustico-jugulare (ch. 60/0); loss of pterygoid-basioccipital contact (ch. 
61/0); distinct anal notch on plastron present (ch. 124/1); one single gular scale present (ch. 128/1); inframarginal scales absent (ch. 139/1); formed central articulation of cervical vertebrae (ch. 145/1); and sutural articulation of pelvis to shell present (ch. 168/1). Although the reduction of the posterior process of the pterygoid bracing the braincase (ch. 60/0) may seem counterintuitive (see character 47, above), Joyce (2007: 72) already suggested that the pleurodire condition (braincase braced by the quadrate and prootic) probably evolved from the 'cryptodire' condition (braincase braced by the pterygoid), which is also found in some stem turtles (e.g., Meiolania platyceps, Mongolochelys efremovi and Kallokibotion bajazidi). Within panpleurodires, Platychelys oberndorferi, Caribemys oxfordiensis and Notoemys laticentralis form a clade which is the sister group of Pleurodira (crowngroup). The former group is unambiguously supported by the following synapomorphies: vertebral scale 3-4 sulcus positioned on neural 6 (ch. 107/0); and retention of a central plastral fontanelle in adult individuals (ch. 111/1). Within this clade, Platychelys oberndorferi and Caribemys oxfordiensis share the presence of a narrow and posteriorly pointed articular site of the ilium with the carapace (ch. 170/0). Pleurodira (sensu Joyce et al. 2004) are supported by the following unambiguous synapomorphies: ventromedial process of the quadrate and/or prootic that floors the cavum acustico-jugulare present (ch. 47/1); incisura columellae auris closed by the quadrate (ch. 53/1); and vertebrals 2 to 4 as narrow, or narrower than pleurals (ch. 106/1).

\section{Paracryptodira Gaffney, 1975 a (sensu Lipka et al. 2006)}

As discussed above, basal relationships between panpleurodires, paracryptodires and eucryptodires are not resolved by the present data set. The only relationships that is unambiguously supported is expressed in SRC tree 24 (Fig. 9): pleurosternids and baenids are more closely related to each other than either is to 'plesiochelyids', but their relationships with panpleurodires, all remaining eucryptodires, Sichuanchelys chowi, 'Chengyuchelys' dashanpuensis and chengyuchelyids are unstable. This is expressed in the Adams consensus tree by common nestings (see Paracryptodira and 'Plesiochelyidae' in Fig. 8B), which should not be confounded with clades.

In the Adams consensus tree, paracryptodires are united by the following unambiguous synapomorphies: dorsal prefrontal exposure reduced to small lappet (ch. 10/1); and foramen posterius canalis carotici interni positioned halfway along the suture between the basisphenoid and the pterygoid (ch. 80/1). The phylogenetic position of Dorsetochelys delairi within paracryptodires is unclear in the present analysis (Fig. 8B). SRC tree 18 (Fig. 9) indicates that the data set strictly supports a close relationship of Dorsetochelys delairi with pleurosternids, but panpleurodires, baenids, Sichuanchelys chowi, 'Chengyuchelys' dashanpuensis and chengyuchelyids are unstable with respect to this relationship. When extant pleurodires are discarded, Dorsetochelys delairi is found within Pleurosternidae, whereas Dinochelys whitei has ambiguous relationships with other paracryptodires (Fig. 10B).

\section{Pleurosternidae Cope, 1868}

Pleurosternids are supported by the following unambiguous synapomorphies: jugal excluded from orbital margin by postorbital-maxilla contact (ch. 28/1); and marginal scales overlap onto costal plates (ch. 104/1). This node consists of a polytomy between Arundelemys dardeni, Dinochelys whitei and Pleurosternon bullockii + Glyptops plicatulus (Fig. 8B). The data set strictly supports that these four species are more closely related to each other than either is to remaining turtles, but Dorsetochelys delairi, Sichuanchelys chowi and 'Chengyuchelys' dashanpuensis have unstable relationships with respect to this group (SRC tree 11; Fig. 9). SRC tree 12 indicates that D. whitei has ambiguous relationships within pleurosternids (Fig. 9) and that the data set strictly supports a topology where P. bullockii and G. plicatulus are more closely related to each other than either is to $A$. dardeni. This study differs from Lipka et al. (2006) in finding $A$. dardeni within pleurosternids, whereas it was considered a paracryptodire more basal than the clade Pleurosternidae + Baenidae by the latter authors. The node Pleurosternon bullockii + Glyptops plicatulus is unambiguously supported by the following synapomorphies in the Adams consensus: contact between basisphenoid and vomer (ch. 65/1); reacquisition of basipterygoid processes (ch. 74/0); and vertebral 5 not overlapping onto peripheral or pygal (ch. 109/1).

\section{Baenidae Cope, 1882}

Baenids are supported by two unambiguous synapomorphies: epipterygoid absent (ch. 55/1); and vertebral scales 2 to 4 as narrow as, or narrower than, pleurals (ch. 106/1). SRC tree 5 (Fig. 9) shows that Chengyuchelys baenoides has unstable relationships with respect to baenids and alone prevents the graphical representation of this clade in the strict consensus tree. Baenids to the exclusion of Neurankylus eximius are unambiguously supported by the absence or near absence of exposure of the prefrontal on the skull roof (ch. 9/1), and by the presence of anal scales that overlap 
A

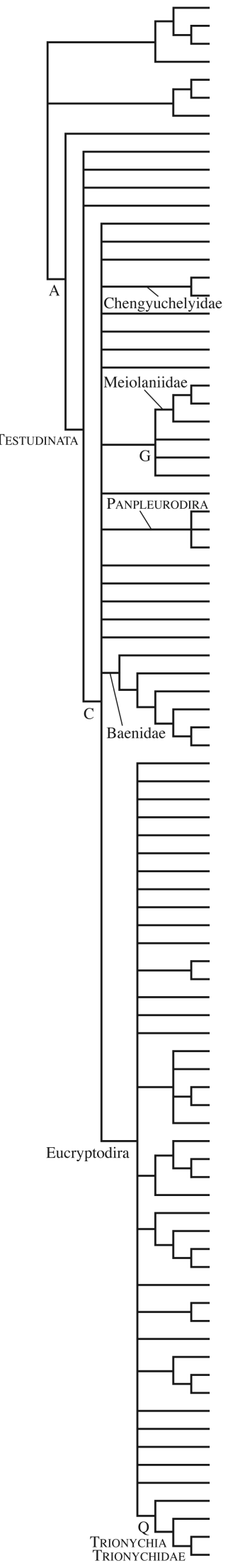

Anthodon serrarius

Araeoscelidia

Captorhinidae

Owenetta kitchingorum

Simosaurus gaillardoti

Protorosaurus speneri

Protorosaurus speneri
Gephyrosaurus bridensis

Gephyrosaurus bridensis
Odontochelys semitestacea

Odontochelys semitestacea
Proterochersis robusta

Proganochelys quensted

Palaeochersis talampayensis

Australochelys africanus

Kayentachelys aprix

Indochelys spatulata

Sichuanchelys chowi

Chengyuchelys baenoides

Chengyuchelys zigongensis

'Chengyuchelys' dashanpuensis

Eileanchelys waldmani

Heckerochelys romani

Condorchelys antiqua

Meiolania platyceps

Ninjemys oweni

Niolamia argentina

Mongolochelys efremovi

Otwayemys cunicularius

Naomichelys speciosa

Kallokibotion bajazidi

Platychelys oberndorferi

Caribemys oxfordiensis

Notoemys laticentralis

Arundelemys dardeni

Pleurosternon bullockii

Glyptops plicatulus

Dorsetochelys delairi

Dinochelys whitei

Neurankylus eximiu

Trinitichelys hiatti

Plesiobaena antiqu

Boremys pulchra

Boremys pulchra

Baena arenosa

Chisternon undatum
Plesiochelys solodurensis

Plesiochelys solodurensis
Portlandemys mcdowelli

Portlandemys mcdowell
'Thalassemys moseri'

Solnhofia parsonsi

Santanachelys gaffneyi

Chubutemys copelloi

Xinjiangchelys qiguensis

Siamochelys peninsularis

Xinjiangchelys tianshanensis

Xinjiangchelys latimarginalis

Annemys levensis

Sinemys lens

Ordosemys leios

Dracochelys bicuspis

Hangaiemys hoburensis

Judithemys sukhanovi

Judithemys sukhanovi
Toxochelys latiremis

Mesodermochelys undulatus

Caretta caretta

Chelonia mydas

Dermochelys coriacea

Protochelydra zangerli

Macrochelys temminckii

Chelydra serpentina

Platysternon megacephalum

Mongolemys elegans

Chrysemys picta

Chrysemys picta
Gopherus polyphemus

Gopherus polyphemus

Geoclemys hamiltonii

Emarginachelys cretacea

Baptemys wyomingensis

Dermatemys mawii

Hoplochelys crassa

Staurotypus triporcatus

Sternotherus odoratus

Kinosternon flavescens

Adocus beatus

Basilemys variolosa

Zangerlia neimongolensi

Sandownia harrisi

Peltochelys duchastelli

Pseudanosteira pulchra

Carettochelys insculpta

Apalone ferox

Lissemys punctata $\neg$ B
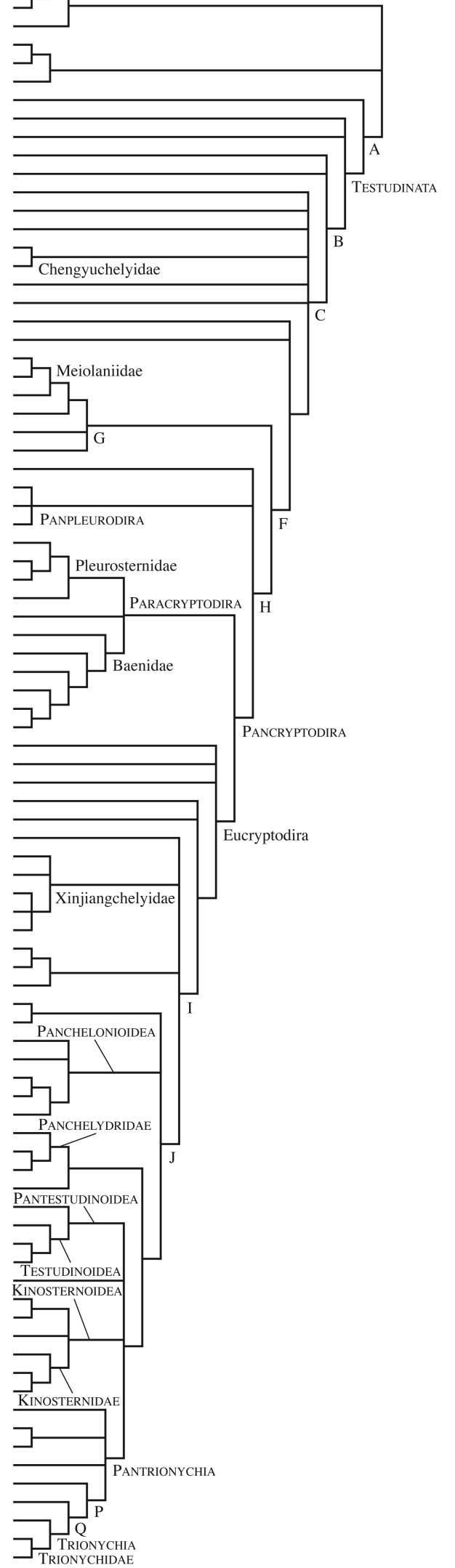

Figure $10-$ Phylogenetic interrelationships of turtles after exclusion of extant pleurodires prior to analysis. (A) strict consensus (591 steps; $\mathrm{Cl}=0.2690 ; \mathrm{Rl}=0.6317$ ) of 641 fundamental trees; (B) Adams consensus (410 steps; $\mathrm{Cl}=0.3878 ; \mathrm{RI}=0.7860$ ) of 641 fundamental trees. Names in small capitals are defined phylogenetically. Capital letters label unnamed nodes. Numbers on the strict consensus tree correspond to Bremer support values. 
anteromedially onto the hypoplastron (ch. 138/1). Hence, the Adams consensus supports the hypothesis that the reduced prefrontal lappets found in Neurankylus eximius are homologous to those found in pleurosternids and that the condition in more derived baenids, in which the dorsal exposure of the prefrontal is absent, evolved from this morphology.

Relationships within the clade Baenidae may be misinterpreted from the Adams consensus tree (Fig. 8B). The nesting Plesiobaena antiqua + Boremys pulchra is not monophyletic and its presence in the Adams consensus tree simply indicates that these two species share a common ancestor that is distinct from the polytomous node uniting them to Trinitichelys hiatti and Baena arenosa + Chisternon undatum. SRC trees 5 reveals the relationships within baenids as strictly supported by the data set: Neurankylus eximius is more basal than a polytomy formed by Trinitichelys hiatti, Plesiobaena antiqua, Boremys pulchra, and Baena arenosa + Chisternon undatum (Fig. 9). When extant pleurodires are discarded prior to the analysis, the clade Baenidae is present in the strict consensus tree and interrelationships within baenids are entirely resolved (Fig. 10A).

\section{'Plesiochelyidae' Rütimeyer, 1873}

An assemblage of Late Jurassic turtles from Western Europe is usually considered to represent the most basal eucryptodires. These forms are referred to families Plesiochelyidae, Eurysternidae and Thalassemydidae, yet their systematics is actually rather problematic. As a result, these forms are generally given little attention in phylogenetic analyses. Joyce (2007) was indeed the first computed analysis including several of these forms as separate terminals (Plesiochelys solodurensis, Portlandemys mcdowelli, 'Thalassemys moseri' and Solnhofia parsonsi).

'Plesiochelyidae' are not monophyletic in the present study, despite the reconstruction provided in the Adams consensus tree (Fig. 8B). As illustrated above for baenids, this is a misleading interpretation inherent to this particular consensus method. SRC tree 23 reveals that this group can be represented graphically only if all other eucryptodires and panpleurodires are pruned from the tree (Fig. 9). This indicates that basal relationships among Testudines are unclear (see Testudines, above) and that plesiochelyids are probably paraphyletic with respect to remaining eucryptodires. Similarly, the clade Solnhofia parsonsi + Santanachelys gaffneyi can only be represented graphically when Portlandemys mcdowelli, 'Thalassemys moseri' and all more derived eucryptodires are pruned from the tree (see SRC tree 22 in Fig. 9). Detailed analyse of the SRC trees further indicates that Portlandemys mcdowelli has unstable relationships among eucryptodires, especially with Xinjiangchelyidae (see SRC tree 10 in Fig. 9). Hence, relationships of 'plesiochelyids' remain largely uncertain in the present analysis. When extent pleurodires are discarded in an attempt to gain some resolution at the Testudines node (see above), 'Plesiochelyidae' are a paraphyletic group at the base of the eucryptodire clade (Fig. 10B).

The present study confirms the results of Joyce (2007) regarding the placement of Santanachelys gaffneyi as a basal eucryptodire rather than as a member of Chelonioidea. Protostegidae like S. gaffneyi are usually considered to be the sister group of Dermochelyidae, the clade that contains the extant leatherback turtle Dermochelys coriacea (Hirayama 1998; Kear \& Lee 2006). More protostegids should be included in future studies in order to confirm this relationship with basal eucryptodires (see also character 81, above).

\section{Node I}

This node unites all eucryptodires to the exception of 'plesiochelyids' based on the following unambiguous synapomorphies: parietal-squamosal contact absent (ch. 16/1); Eustachian tube not contained within incisura columellae auris alongside stapes (ch. 54/0); crista supraoccipitalis protruding significantly posterior to foramen magnum (ch. 68/1); foramen posterius canalis carotici interni formed by the pterygoid near the posterior edge of this bone (ch. 81/1); vertebrals 2 to 4 as narrow, or narrower than, pleurals (ch. 106/1); ligamentous bridge (ch. 110/1); axillary buttresses contacting peripheral only (ch. 119/0); mesoplastron absent (ch. 120/1); inguinal buttresses contacting peripherals only (ch. 123/0); and transverse processes of cervical vertebrae in the anterior part of the centra (ch. 142/1). Because of the spurious reconstruction of the Adams consensus tree regarding 'plesiochelyids', some of the aforementioned characters (e.g., chs 81, 106, 120) may actually represent synapomorphies of more inclusive clades. SRC tree 17 (Fig. 9) indicates that this clade uniting all eucryptodires more derived than 'plesiochelyids' is strictly supported by the data, but that panpleurodires and Portlandemys mcdowelli have unstable relationships with respect to this group.

Node I consists of a polytomy between Xinjiangchelyidae, Dracochelys bicuspis, Sinemys lens + Ordosemys leios, and node J (Fig. 8B). It does not represent a 'true' polytomy: $S$. lens $+O$. leios and D. bicuspis actually have unstable relationships. The clade $S$. lens +0 . leios, itself strictly supported by the data set (Fig. 8A), shows unstable relationships with respect to the next less inclusive node, which unites Hangaiemys hoburensis, Judithemys sukhanovi and Cryptodira (see SRC tree 14 in 
Fig. 9). The relationships of $D$. bicuspis are even more unstable, especially with respect to Xinjiangchelyidae, other 'sinemydids' - 'macrobaenids', and Panchelonioidea (see SRC trees 6, 10, 14, 15 and 19 in Fig. 9). All in all, relationships of 'sinemydids' - 'macrobaenids' (S. lens, $O$. leios, D. bicuspis, $H$. hoburensis and J. sukhanovi) remain unclear after the present analysis.

In the present analysis, the position of the Eustachian tube relative to the incisura columellae auris is tentatively coded separately from the closure of the incisura by the quadrate (see characters 53 and 54, above). This allows these two features to be tested independently for congruence. In Joyce (2007, ch. 36), these features were coded as a single unordered multistate character with the absence/presence of the incisura columellae auris. According to Joyce (2007), a closed incisura enclosing only the stapes appeared twice (in Sinemys lens and the node formed by chelydrids, testudinoids and trionychoids) and was lost three times (in Adocus beatus, kinosternoids, and the clade formed by Chrysemys picta and Geoclemys hamiltonii), while a closed incisura including the Eustachian tube evolved three times (in Meiolania platyceps, Kallokibotion bajazidi, and pleurodires). In the present analysis, a closed incisura (including the Eustachian tube or not; ch. 53) appears to be a rather homoplastic character with six independent acquisitions (ci = 0.167): in Kallokibotion bajazidi, Pleurodira, Sinemys lens, Gopherus polyphemus, panchelydrids + Platysternon megacephalum, and pantrionychians more derived than Adocus beatus. The present analysis also supports the hypothesis that the Eustachian tube is contained within the incisura columellae auris (closed or not; ch. 54) as a plesiomorphic condition for turtles and that this condition is lost only once ( $\mathrm{ci}=1$ ) as an unambiguous synapomorphy of node I (i.e., Xinjiangchelyidae + 'macrobaenids' - 'sinemydids' + Cryptodira). The present coding strategy allows to propose a more logical evolutionary scenario: the closing of the incisura columellae auris evolved independently several times within turtles (probably for various purposes) and the Eustachian tube is usually contained within the incisura along the stapes, with the exception of a very inclusive group of eucryptodires (node I). The exclusion of the Eustachian tube from the incisura columellae auris in node $\mathrm{I}$ is a single evolutionary event. In all species with a closed incisura (independently evolved) above node I, the Eustachian tube is not contained within the incisura, whereas in all species with a closed incisura (also independently evolved) below that node, the Eustachian tube is contained within the incisura along the stapes. However, because no pancryptodire below node $\mathrm{I}$ is known to have a closed incisura this evolutionary event could be a synapomorphy of a more inclusive clade of pancryptodires. This hypothesis regarding the position of the Eustachian tube could be tested by comparing softtissue anatomy of extant pleurodires and that of extant cryptodires. Siebenrock (1897) already noted that extant pleurodires have the Eustachian tube within the incisura when this latter is closed by bone, whereas in extant cryptodires the Eustachian tube is excluded from the closed incisura. However, it should be investigated whether or not anatomical discrepancies relative to the position of the Eustachian tube exist between pleurodires and cryptodires that have an open incisura columellae auris.

\section{Xinjianchelyidae Ye, 1986}

Xinjiangchelyids are supported by the following unambiguous synapomorphies: distinctly sinuous midline sulcus of plastral scales (ch. 127/1); and gular scales restricted to epiplastra only (ch. 129/1). SRC tree 10 indicates that Portlandemys mcdowelli and Dracochelys bicuspis have unstable relationships with respect to xinjiangchelyids (Fig. 9). This clade consists of a polytomy between Siamochelys peninsularis, Xinjiangchelys qiguensis and the trichotomy $X$. latimarginalis - $X$. tianshanensis Annemys levensis (Fig. 8B). The latter clade is unambiguously supported by the shared presence of anal scales that overlap onto the hypoplastra (ch. 138/1). SRC trees do not help to resolve the relationships among xinjiangchelyids further, but SRC tree 10 confirms the above topology as being that which is strictly supported by the data set (Fig. 9).

One of the most interesting results of this study is the placement of Siamochelys peninsularis within xinjiangchelyids. This is the first time that the phylogenetic relationships of this Middle Jurassic species from Thailand are investigated. In their original description, Tong et al. (2002) suggested that Siamochelys peninsuralis was closely related to the genera Chengyuchelys and Xinjiangchelys, but these may not be closely related according to the present study.

\section{Sinemys lens + Ordosemys leios}

This clade is unambiguously supported by the following synapomorphies: medial contact of prefrontals absent (ch. 5/0); dorsal exposure of prefrontals reduced to small lappets (ch. 10/1); precolumellar fossa present (ch. 50/1); paired pits on ventral surface of basisphenoid present (ch. 73/1); and central plastral fontanelle present (ch. 111/1). The exact relationships of this clade, which is strictly supported by the data set (Fig. 8A), with other 'sinemydids' - 'macrobaenids' and Cryptodira remain uncertain (see Node l, above). 


\section{NodeJ}

This node consists of a polytomy between Hangaiemys hoburensis + Judithemys sukhanovi, Panchelonioidea, Mongolemys elegans and node K (Fig. 8B). Node J is supported by the following unambiguous synapomorphies: extragulars absent (ch. 130/1); posterior cervical vertebrae with strongly developed ventral keels (ch. 143/1); and eighth cervical centrum significantly shorter than seventh (ch. 144/1). SRC tree 14 (Fig. 9) indicates that this clade, which groups $H$. hoburensis and J. sukhanovi with Cryptodira, is strictly supported by the data set, but that Portlandemys mcdowelli, Dracochelys bicuspis, Sinemys lens and Ordosemys leios are unstable with respect to this relationship. In other words, relationships of 'sinemydids' - 'macrobaenids' remain unclear, and it should not be concluded from the present analysis that $H$. hoburensis and J. sukhanovi are more closely related to Cryptodira than to other 'sinemydids' - 'macrobaenids'. In fact, the five species representing 'sinemydids' - 'macrobeanids' in the present data set are unstable with respect to Cryptodira (see SRC tree 15 in Fig. 9).

\section{Hangaiemys hoburensis + Judithemys sukhanovi}

This nesting is unambiguously supported by the presence of paired pits on the ventral surface of the basisphenoid (ch. 73/1) and the elongate shape of epiplastra (ch. 118/1) in the Adams consensus tree (Fig. $8 \mathrm{~B})$. However, this group is not strictly supported as monophyletic by the data set: SRC tree 19 (Fig. 9) indicates that $H$. hoburensis and J. sukhanovi are more closely related to each other than either is to xinjiangchelyids, 'plesiochelyids' or any more basal turtle, but their relationships with other 'sinemydids' 'macrobaenids' or with Cryptodira remain uncertain.

\section{Cryptodira Cope, 1868 (sensu Joyce et al. 2004)}

Relationships between major groups of Cryptodira are not well resolved in the present analysis. The topology of the Adams consensus tree is particularly deceitful in that matter (Fig. 8B): nodes labelled $\mathrm{K}$ and $\mathrm{L}$ are actually not strictly supported by the data set. Hence, nodes $\mathrm{K}$ and $\mathrm{L}$ should not be regarded as statements of relationships and can be collapsed in order to obtain a better idea of strictly supported relationships.

Relationships of Mongolemys elegans remain unclear after the present analysis (Figs 8 and 9). However, when extant pleurodires are discarded, $M$. elegans is strictly supported as a stem testudinoid (Fig. 10A), as suggested by Sukhanov (2000).

\section{Panchelonioidea Joyce et al., 2004}

Panchelonioids are supported by six unambiguous synapomorphies: raised pedestal on the visceral surface of the nuchal for the articulation with the neural spine of the eighth cervical vertebra (ch. 90/1); reduction of costal ossifications, costal fontanelles well-developed (ch. 101/1); central plastral fontanelle retained in adult individuals (ch. 111/1); epiplastra elongate in shape (ch. 118/1); xiphiplastra reduced to narrow struts that frame a xiphiplastral fontanelle (ch. 125/1); and paddles present (ch. 177/1). SRC tree 6 (Fig. 9) reveals that the clade Panchelonioidea is strictly supported by the data set, but that Dracochelys bicuspis has unstable relationships with respect to it.

In the Adams consensus tree (Fig. 8B), Toxochelys latiremis and Mesodermochelys undulatus form a polytomy at the base of panchelonioids. SRC trees 7 and 8 (Fig. 9) respectively indicate that $T$. latiremis has unstable relationships with respect to other panchelonioids and that $M$. undulatus has unstable relationships regarding chelonioids. The nesting formed by the three extant chelonioids in the Adams consensus tree actually represents a strictly supported three-taxon statement, but the placement of $M$. undulatus with respect to these three taxa is uncertain (SRC tree 8). Mesodermochelys undulatus is usually considered to be a close relative of Dermochelys coriacea (e.g., Hirayama \& Chitoku 1996; Kear \& Lee 2006). In Joyce (2007), this relationship was only supported by the partial loss of carapacial scales, an ordered multistate character (see character 87, above). When characters were run unordered (first analysis of Joyce 2007), the relationship between $M$. undulatus and $D$. coriacea was no longer supported, as in the present analysis.

Chelonioidea (sensu Joyce et al. 2004) are supported by the following synapomorphies: parietal-squamosal contact present, temporal emargination poorly developed (ch. 16/0); foramen palatinum posterius absent (ch. 63/1); and rostrum basisphenoidale rod-like, thick and rounded (ch. 72/1). According to the present analysis, the loss of the foramen palatinum posterius (fpp) in chelonioids derived from a condition where the $\mathrm{fpp}$ is entirely surrounded by bone, which justifies a posteriori the coding of two independent characters for the loss of the $\mathrm{fpp}$ and the presence of a laterally open fpp in some 'plesiochelyids' (see characters 63 and 64, above). Among chelonioids, Chelonia mydas and Caretta caretta share the following unambiguous synapomorphies: foramen praepalatinum absent (ch. 34/1); and anterior articulation of first dorsal centrum facing strongly anteroventrally (ch. 157/1). 


\section{Node $K$}

As discussed above (see Cryptodira), this node is not strictly supported by the data set (not present in any SRC tree). In the Adams consensus tree (Fig. 8B), this is the representation of a common nesting that does not provide any unambiguous information on the relationships between included and excluded taxa. In this context, this nesting, which groups Testudinidae, Panchelydridae + Platysternon megacephalum and node $\mathrm{L}$, is supported by the following synapomorphies: pterygoid contribution to foramen palatinum posterius absent (ch. $66 / 1)$; and anterior articulation of first dorsal centrum facing strongly anteroventrally (ch. 157/1).

\section{Testudinoidea Fitzinger, 1826 (sensu Joyce et al. 2004)}

The clade Testudinoidea (Fig. 8A) is unambiguously supported by the following synapomorphies: axillary buttress contacts peripherals and first costal (ch. 119/1); inguinal buttress contacts peripherals and costals (ch. 123/1); and only two pairs of inframarginal scales present (ch. 140/1).

\section{Panchelydridae + Platysternon megacephalum}

The present analysis unambiguously supports the placement of Platysternon megacephalum as the sister group of panchelydrids (Fig. 8A). A close relationship between $P$. megacephalum and Chelydridae has been proposed many times in the past (see Gaffney 1975b), but P. megacephalum was generally considered to be a derived chelydrid (e.g., Gaffney 1975b; Gaffney \& Meylan 1988). However, Shaffer et al. (1997) and Brinkman \& Wu (1999) have suggested a sister group relationship between these two taxa. These results are in contradiction with the most recent molecular studies which optimised Platysternon megacephalum to be a member of testudinoids (Parham et al. 2006), as well as with Joyce (2007) for which $P$. megacephalum is more closely related to testudinoids and trionychoids than to panchelydrids. New data on the morphology of the respiratory system in turtles also support a close relationship between $P$. megacephalum and some testudinoids, Emydidae particularly (Lambertz et al. 2010).

In the present study, the clade uniting panchelydrids and $P$. megacephalum is supported by the following unambiguous synapomorphies: frontal excluded from the orbital margin (ch. 13/0); incisura columellae auris closed by quadrate (ch. 53/1); epiplastra elongate in shape (ch. 118/1); and chevrons present (ch. 158/0).

\section{Panchelydridae Joyce et al., 2004}

Panchelydrids are supported by the following unambiguous synapomorphies: medial contact of abdominal scales absent (ch. 137/1); and anal scales overlap medially onto hypoplastra (ch. 138/1). In the context of the present analysis, chelydrids (i.e., Chelydra serpentina + Macrochelys temminckii) are supported unambiguously only by the retention of a central plastral fontanelle in adult individuals (ch. 111/1).

\section{Node $L$}

As node $\mathrm{K}$ (see above), this node is not strictly supported by the data set (not present in any SRC tree). In the Adams consensus tree (Fig. 8B), this is the representation of a common nesting that does not provide any unambiguous information on the relationships between included and excluded taxa. In this context, this nesting, which groups Emarginachelys cretacea, Kinosternoidea and Pantrionychia, is supported by the shared presence of a palatine contribution to the anterior extension of the lateral braincase wall (ch. 46/1). SRC tree 9 (Fig. 9) reveals that E. cretacea has unstable relationships with kinosternoids.

\section{Kinosternoidea Joyce et al., 2004}

Kinosternoids are supported by the following unambiguous synapomorphies: pectoral scales absent (ch. 135/1); and articulation between cervical vertebrae 3 and 4 concave anteriorly (i.e., 3)4; ch. 147/1). The interrelationships within Kinosternoidea exhibited in the Adams consensus tree correspond to those that are strictly supported by the data set, as indicated by SRC tree 9 (Fig. 9). Hence, relationships between Hoplochelys crassa, Dermatemys mawii + Baptemys wyomingensis, and Kinosternidae are ambiguous ('true' polytomy; Fig. 8B).

\section{Pantrionychia Joyce et al., 2004}

Relationships within pantrionychians are fully resolved by the present data set (Fig. $8 \mathrm{~A}$ ). This clade is supported by the following unambiguous synapomorphies: distincly sinuous midline sulcus of plastral scales (ch. 127/1); reacquisition of extragulars (ch. 130/0); and central articulations between cervical vertebrae 4-5, 5-6 and 6-7 convex anteriorly (chs 148/0, $149 / 0$ and 150/0). Adocus beatus is found to be the most basal member of the group and to be the sister taxon of a clade uniting the remaining pantrionychians (i.e., node M). 


\section{Node $M$}

This clade is supported by the following unambiguous synapomorphies: vomer-pterygoid contact in palatal view absent (ch. 44/1); and incisura columellae auris closed by the quadrate (ch. 53/1). Clade M splits basally into two clades: clade $\mathrm{N}$, containing Basilemys variolosa and Zangerlia neimongolensis, and clade $\mathrm{P}$, containing Peltochelys duchastelli and Trionychia.

\section{Node $N$}

Clade $\mathrm{N}$ is supported by the following unambiguous synapomorphies: extragular scales contacting one another posterior to gulars (ch. 131/1); and most digits with two short phalanges (ch. 176/1). The content of this clade is rather unexpected. While Basilemys variolosa and Zangerlia neimongolensis are usually considered as basal pantrionychians, the placement of Sandownia harrisi and especially that of Chubutemys copelloi are surprising. Chubutemys copelloi is known from relatively poorly preserved material from the Early Cretaceous of Argentina. It was interpreted by Gaffney et al. (2007) as closely related to meiolaniids. This species is sparsely scored in the present analysis (approximately 70\% missing data) and its relationships are likely to change in future studies, owing especially to the ongoing description of new material (J. Sterli, pers. comm. 2009). The exclusion of extant pleurodires from the analysis is enough to take $C$. copelloi out of pantrionychians (Fig. $10 \mathrm{~B})$. In this case, SRC trees reveal that this species is unstable with respect to relationships among cryptodires more derived than panchelonioids.

The present analysis agrees with Meylan et al. (2000) in the placement of Sandownia harrisi as a member of Trionychoidea (sensu Joyce et al. 2004). In contrast, Joyce (2007: 66) tentatively reconstructed Sandownia harrisi as the sister taxon of 'Thalassemys moseri' because this hypothesis only required one additional step compared to its placement as a basal trionychoid. In the present analysis, Sandownia harrisi is unambiguously supported as a member of pantrionychians (see below), although its phylogenetic relationships are prone to change with future discoveries as this species is only known from cranial material while most characters supporting relationships within pantrionychians concern shell or postcranial morphology.

\section{Node 0}

This clade is supported by the presence of a parietalsquamosal contact (ch. 16/0). The clade Zangerlia neimongolensis + Chubutemys copelloi is supported by the absence of a frontal contribution to the orbital margin (ch. 13/0).

\section{Node $P$}

This clade is unambiguously supported by a reduction of the number of peripherals to 10 pairs (ch. 98/1).

\section{Node $Q$}

This clade is supported by the following unambiguous synapomorphies: reduction of posterior neurals allowing partial contact of posterior costals (ch. 94/1); ligamentous connection between carapace and plastron (ch. 110/1); and plastral scales absent (ch. 126/1) In the present analysis, Pseudanosteira pulchra is the sister taxon of Trionychia (Fig. 8). In other analyses, P. pulchra is rather considered to be a carretochelyid. In Joyce (2007) for example, the clade formed by Carettochelys insculpta and $P$. pulchra was only supported by the development of elongate flippers, a character that is not considered in the present analysis (see Supplementary Material). More characters supporting carettochelyids should be included in future analyses.

\section{Trionychia Hummel, 1929 (sensu Joyce et al. 2004)}

Trionychians are unambiguously supported by the absence of carapacial scales (ch. 87/1). The placement of Apalone ferox is reconstructed from STR results (see above). Trionychidae would be supported at least by the following synapomorphies: exclusion of the premaxilla from the apertura narium externa (ch. 36/1); and strap like and V-shaped entoplastron (ch. 115/1).

\section{Conclusions}

The present study builds upon the analysis of Joyce (2007) by adding 19 fossil turtle species to the taxon sample in order to achieve a more thorough representation of basal taxa. Additional characters have been taken from previous studies or personal observations, and several characters have been recoded in order to maximise the testing of primary homology statement by congruence. The resulting topology generally agrees with that of Joyce (2007) and similar levels of resolution are attained. With the exception of Sichuanchelys chowi, 'Chengyuchelys' dashanpuensis, and chengyuchelyids, the phylogenetic relationships of newly added species have been resolved by the present analysis.

In agreement with Li et al. (2008), Odontochelys semitestacea is found to be the most basal turtle known to date. In contrast to most previous analyses, the 


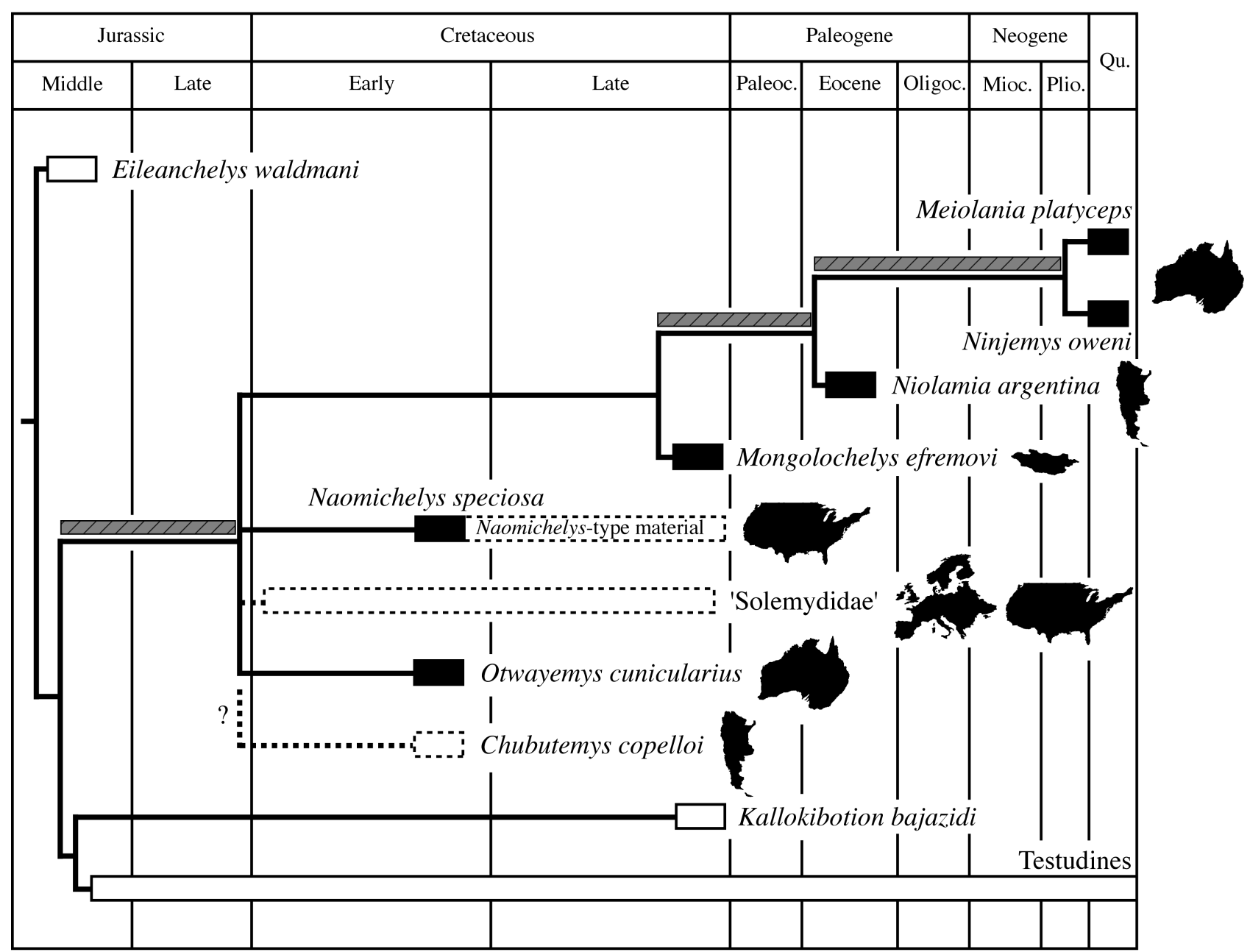

Figure 11 - Stratigraphic and geographic repartition of meiolaniids and related taxa. In addition to taxa included in the present study (black), the stratigraphical extent of Naomichelys-type material in North America (W. G. Joyce, pers. comm. 2010) and that of 'Solemydidae' in Europe and North America (Lapparent de Broin 2001) is indicated with dashed lines. Following Gaffney et al. (2007), Chubutemys copelloi is also represented as possibly related to meiolaniids. Hatched grey bars represent major gaps in the fossil record of meiolaniids and related taxa.

relationships of Proterochersis robusta are ambiguous in the present study. This undoubtedly results from the inclusion of Odontochelys semitestacea, which also possesses two pairs of mesoplastra, while other features of Proterochersis robusta suggest that it is more derived than Proganochelys quenstedti and Palaeochersis talampayensis. As in Sterli (2008), Indochelys spatulata form a polytomy with Kayentachelys aprix, confirming the original conclusions of Datta et al. (2000). In contrast to Anquetin et al. (2009), Heckerochelys romani and Condorchelys antiqua are found to be more basal than Eileanchelys waldmani. Although the phylogenetic position of chengyuchelyids remains somewhat uncertain, this study suggests that they may be stem turtles. This would be in contradiction with their usual interpretation as basal pancryptodires more or less closely related to xinjiangchelyids (e.g., Young \& Chow 1953; Ye 1994; Danilov \& Parham 2008), but further investigation is needed on this point. In the present analysis, Arundelemys dardeni is nested within pleurosternids rather than being more basal than the pleurosternid-baenid dichotomy as previously suggested (Lipka et al. 2006). This is more in agreement with available data: e.g., presence of a maxillapostorbital contact. In contrast, the placement of Dorsetochelys delairi remains unclear. The Middle Jurassic turtle Siamochelys peninsularis, which is included in a phylogenetic analysis for the first time, is nested within xinjiangchelyids. As previously suggested by Matzke et al. (2004), the genus Xinjiangchelys is paraphyletic and nomenclatural adjustments are necessary. However, a proper reassessment of the xinjiangchelyids is needed and recently found material in China, including cranial remains, will undoubtedly form the basis for such a revision (Wings \& Joyce 2009). Because the present data set is largely based on that of Joyce (2007), it is not surprising that the phylogenetic position of Panpleurodira 
is also problematic herein. Convergence between extant pleurodires and certain pancryptodires and the lack of fossil panpleurodires obscure basal relationships within crown-group turtles. When extant pleurodires are excluded from the data set, a more conventional topology emerges (Fig. 10), but the exact relationships of panpleurodires remains uncertain. Only the scoring of more panpleurodires and basal pleurodires could help to resolve the relationships of this clade among other turtles.

The primary purpose of the present analysis was not to improve the resolution within Testudines and no effort has been made to include more characters or more taxa in this matter. However, two conclusions can be drawn for them. Firstly, the alternative coding strategy followed herein, which does not consider transformation series, results in a minor resolution of relationships, especially among extant forms. This underlines the need for more clear-cut characters not relying on individual interpretation of possible transformation series. Secondly, although most authors and most studies agree on the broad-scale relationships among eucryptodires ('plesiochelyids' more basal than xinjiangchelyids, themselves more basal than 'sinemydids' 'macrobaenids'), it is evident that finer relationships are poorly understood and require a significant revision. In addition to these remarks and as discussed above, most analyses also suffer from a poor representation of panpleurodires, which is only partly due to a lack of material. In general, studies of turtle interrelationships would benefit from a greater consideration of fossil species and a lesser reliance on extant forms.

The most interesting perspective of the present work consists in the affinities of meiolaniids and related species. As in Hirayama et al. (2000) and Joyce (2007), Mongolochelys efremovi is recovered as the sister taxon of meiolaniids, and, as in Joyce (2007), these forms are placed along the stem of Testudines. The present analysis also supports that Otwayemys cunicularius and Naomichelys speciosa are closely related to Mongolochelys efremovi and meiolaniids, and are thereby stem turtles as well (Fig. 8). This result indicates that a clade of rather large stem turtles persisted alongside crown-group turtles up until the Pleistocene and was actually spread worldwide at least during the Mesozoic and early Cenozoic, with representatives in South America, North America, Mongolia and Australia (Fig. 11). This clade was also probably present in Europe because Naomichelys speciosa is usually related to the solemydids Solemys and Helochelydra from the Cretaceous of western Europe (Lapparent de Broin \& Murelaga 1999; Milner 2004). The latter fact makes this result important. If meiolaniids are related not only to Mongolochelys efremovi but also to
Naomichelys speciosa and solemydids as proposed by the present analysis, then the aforementioned clade was a non-negligible component of the Cretaceous turtle diversity (Fig. 11). This bridges some of the gaps in the fossil record of this clade and appeals for a revision of solemydids, which are known throughout the Cretaceous in Western Europe and North America (de Lapparent de Broin 2001).

\section{Acknowledgements}

The following researchers and curators are thanked for enabling access to specimens in their care: $C$. Mehling and E. Gaffney (AMNH), B. Zipfel and B. Rubidge (BP), O. Rauhut and A. López-Arbarello (BSPG), W. Simpson and A. Resetar (FMNH), Z. Zhonghe and W. Yuan (IVPP), J. Müller (MB), C. Schaff (MCZ), J. Gillette (MNA), S. Chapman and A. Milner (NHM), J. O. Ebbestad and J. Peel (PMU), R. Schoch and A. Matzke (SMNS), T. Rowe (TMM), P. Holroyd (UCPM), W. Joyce (YPM), G. Peng and Y. Ye (ZDM). Walter Joyce and an anonymous reviewer provided detailed reviews of this work and their comments significantly helped to improve the quality of the paper. I am indebted to my supervisors, S. Evans and P. Barrett, for their insightful comments and advice throughout my thesis. M. Wilkinson, S. Maidment and $\mathrm{R}$. Butler provided valuable information regarding cladistic methodology. The present study also benefited from discussions on turtle anatomy with W. Joyce and J. Sterli, and from careful review of an earlier version of the manuscript by G. Billet. This work was funded by a NHM PhD studentship and a postdoctoral grant from the Simone and Cino del Duca Foundation (2008 Prize awarded to P. Janvier, MNHN).

\section{References}

Anquetin, J. In press. The anatomy of the basal turtle Eileanchelys waldmani from the Middle Jurassic of the Isle of Skye, Scotland. Earth and Environmental Science Transactions of the Royal Society of Edinburgh.

Anquetin, J., Barrett, P.M., Jones, M.E.H., Moore-Fay, S. \& Evans, S.E. 2009. A new stem turtle from the Middle Jurassic of Scotland: new insights into the evolution and palaeoecology of basal turtles. Proceedings of the Royal Society of London, B, 276, 879-886.

Batsch, A.J.G.C. 1788. Versuch einer Anleitung, zur Kenntniß und Geschichte der Thiere und Mineralien. Akademische Buchhandlung, Jena, 528 pp.

Bonin, F., Devaux, B. \& Dupré, A. 2006. Turtles of the world. John Hopkins University Press, Baltimore, 416 pp. [Translated by P. C. H. Pritchard] 
Brinkman, D.B. \& Nicholls, E.L. 1991. Anatomy and relationships of the turtle Boremys pulchra (Testudines: Baenidae). Journal of Vertebrate Paleontology, 11, 302-315.

Brinkman, D.B. \& Nicholls, E.L. 1993. The skull of Neurankylus eximius (Testudines: Baenidae) and a reinterpretation of the relationships of this taxon. Journal of Vertebrate Paleontology, 13, 273-281.

Brinkman, D.B. \& Wu, X.-C. 1999. The skull of Ordosemys, an Early Cretaceous turtle from Inner Mongolia, People's Republic of China, and the interrelationships of Eucryptodira (Chelonia, Cryptodira). Paludicola, 2, 134-147.

Brinkman, D.B., Stadtman, K. \& Smith, D. 2000. New material of Dinochelys whitei Gaffney, 1979, from the Dry Mesa Quarry (Morrison Formation, Jurassic) of Colorado. Journal of Vertebrate Paleontology, 20, 269-274.

Clark, J. 1932. A new anosteirid from the Uinta Eocene. Annals of the Carnegie Museum, 21, 161-170.

Cope, E.D. 1868. On the origin of genera. Proceedings of the Academy of Natural Science, Philadelphia, 20, 242-300.

Cope, E.D. 1882. Contributions to the history of the Vertebrata of the Lower Eocene of Wyoming and New Mexico, made during 1881. Proceedings of the American Philosophical Society, 20, 139-197.

Danilov, I.G. \& Parham, J.F. 2008. A reassessment of some poorly known turtles from the Middle Jurassic of China, with comments on the antiquity of extant turtles. Journal of Vertebrate Paleontology, 28, 306-318.

Darlu, P. \& Tassy, P. 1993. La reconstruction phylogénétique: concepts et méthodes. Masson, Paris, 244 pp. [http://sfs.snv.jussieu.fr/publications_sfs.shtml]

Datta, P.M., Manna, P., Ghosh, S.C. \& Das, D.P. 2000. The first Jurassic turtle from India. Palaeontology, 43, 99-109.

deBraga, M. \& Rieppel, O. 1997. Reptile phylogeny and the interrelationships of turtles. Zoological Journal of the Linnean Society, 120, 281-354.

Dryden, L.S. 1988. Paraphyly of the Cryptodira and phylogenetic systematics of turtles. Unpublished Master's thesis, University of Kansas, Lawrence, KS.

Evans, J. \& Kemp, T.S. 1975. The cranial morphology of a new Lower Cretaceous turtle from southern England. Palaeontology, 18, 25-40.

Evans, J. \& Kemp, T.S. 1976. A new turtle skull from the Purbeckian of England and a note on the early dichotomies of cryptodire turtles. Palaeontology, 19, 317-324.

Evans, S.E. 1980. The skull of a new eosuchian reptile from the Lower Jurassic of South Wales. Zoological Journal of the Linnean Society, 70, 203-264.

Evans, S.E. 1981. The postcranial skeleton of the Lower Jurassic eosuchian Gephyrosaurus bridensis. Zoological Journal of the Linnean Society, 73, 81-116.

Fang, Q. 1987. A new species of Middle Jurassic turtle from Sichuan. Acta Herpetologica Sinica, 6, 65-69.

Farris, J.S., Kluge, A.G. \& Eckardt, M.J. 1970. A numerical approach to phylogenetic systematics. Systematic Zoology, 19, 172-191.

Fitzinger, L. 1826. Neue Classification der Reptilien nach ihren natürlichen Verwandtschaften. Verlag J.G. Heubner, Wien.

Fox, R.C. \& Bowman, M.C. 1966. Osteology and relationships of Captorhinus aguti (Cope) (Reptilia: Captorhinomorpha). The University of Kansas Paleontological Contributions, Vertebrata, Article 11, 1-79.

Freudenstein, J.V. 2005. Characters, states, and homology. Systematic Biology, 54, 965-973.

Gaffney, E.S. 1972a. An illustrated glossary of turtle skull nomenclature. American Museum Novitates, 2486, $1-33$.

Gaffney, E.S. 1972b. The systematics of the North American family Baenidae (Reptilia, Cryptodira). Bulletin of the American Museum of Natural History, 147, 241-320.

Gaffney, E.S. 1975a. A phylogeny and classification of the higher categories of turtles. Bulletin of the American Museum of Natural History, 155, 389-436.

Gaffney, E.S. 1975b. Phylogeny of the chelydrid turtles: a study of shared derived characters in the skull. Fieldiana - Geology, 33, 157-178.

Gaffney, E.S. 1979a. The Jurassic turtles of North America. Bulletin of the American Museum of Natural History, 162, 91-136.

Gaffney, E.S. 1979b. Comparative cranial morphology of recent and fossil turtles. Bulletin of the American Museum of Natural History, 164, 65-375.

Gaffney, E.S. 1983. The cranial morphology of the extinct horned turtle, Meiolania platyceps, from the Pleistocene of Lord Howe Island. Bulletin of the American Museum of Natural History, 175, 361-479.

Gaffney, E.S. 1984. Historical analysis of theories of chelonian relationship. Systematic Zoology, 33, 283-301. 
Gaffney, E.S. 1990. The comparative osteology of the Triassic turtle Proganochelys. Bulletin of the American Museum of Natural History, 194, 1-263.

Gaffney, E.S. 1996. The postcranial morphology of Meiolania platyceps and a review of the Meiolaniidae. Bulletin of the American Museum of Natural History, 229, 1-166.

Gaffney, E.S. \& Jenkins, F.A. Jr. 2010. The cranial morphology of Kayentachelys, an Early Jurassic cryptodire, and the early history of turtles. Acta Zoologica (Stockholm), 91, 335-368.

Gaffney, E.S. \& Kitching, J.W. 1995. The morphology and relationships of Australochelys, an Early Jurassic turtle from South Africa. American Museum Novitates, 3130, 1-29.

Gaffney, E.S. \& McKenna, M.C. 1979. A Late Permian captorhinid from Rhodesia. American Museum Novitates, 2688, 1-15.

Gaffney, E.S. \& Meylan, P.A. 1988. A phylogeny of turtles. Pp. 157-219 in M. J. Benton (ed.) The Phylogeny and Classification of the Tetrapods, Volume 1: Amphibians, Reptiles, Birds. Clarendon Press, Oxford.

Gaffney, E.S. \& Meylan, P.A. 1992. The Transylvanian turtle, Kallokibotion, a primitive cryptodire of Cretaceous age. American Museum Novitates, 3040, 1-37.

Gaffney, E.S., Hutchison, J.H., Jenkins, F.A. \& Meeker, L.J. 1987. Modern turtle origins: the oldest known cryptodire. Science, 237, 289-291.

Gaffney, E.S., Meylan, P.A. \& Wyss, A.R. 1991. A computer assisted analysis of the relationships of the higher categories of turtles. Cladistics, 7, 313-335.

Gaffney, E.S., Kool, L., Brinkman, D.B., Rich, T.H. \& Vickers-Rich, P. 1998. Otwayemys, a new cryptodiran turtle from the Early Cretaceous of Australia. American Museum Novitates, 3233, 1-28.

Gaffney, E.S., Rich, T.H., Vickers-Rich, P., Constantine, A., Vacca, R. \& Kool, L. 2007. Chubutemys, a new eucryptodiran turtle from the Early Cretaceous of Argentina, and the relationships of the Meiolaniidae. American Museum Novitates, 3599, 1-35.

Gauthier, J., Kluge, A.G. \& Rowe, T. 1988. Amniote phylogeny and the importance of fossils. Cladistics, 4, 105-209.

Gauthier, J., Cannatella, D., de Queiroz, K., Kluge, A.G. \& Rowe, T. 1989. Tetrapod phylogeny. Pp. 337-353 in B. Fernholm, K. Bremer, and H. Jörnvall (eds) The hierarchy of life: molecules and morphology in phylogenetic analysis. Elsevier, Amsterdam.
Goloboff, P. 1999. Analyzing large data sets in reasonable times: solutions for composite optima. Cladistics, 15, 415-428.

Gottmann-Quesada, A. \& Sander, P.M. 2009. A redescription of the early archosauromorph Protorosaurus speneri Meyer, 1832, and its phylogenetic relationships. Palaeontographica, Abteilung A, 287, 123-220.

Hawkins, J.A., Hughes, C.E. \& Scotland, R.W. 1997. Primary homology assessment, characters and character states. Cladistics, 13, 275-283.

Hay, O.P. 1908. The fossil turtles of North America. Carnegie Institution of Washington, Publication, 75, 1-568.

Heaton, M.J. 1979. Cranial anatomy of primitive captorhinid reptiles from the late Pennsylvanian and early Permian Oklahoma and Texas. Oklahoma Geological Survey, Bulletin, 127, 1-84.

Hill, R.V. 2005. Integration of morphological data sets for phylogenetic analysis of Amniota: The importance of integumentary characters and increased taxonomic sampling. Systematic Biology, 54, 530-547.

Hirayama, R. 1998. Oldest known sea turtle. Nature, 392, 705-708.

Hirayama, R. \& Chitoku, T. 1996. Family Dermochelyidae (superfamily Chelonioidea) from the Upper Cretaceous of North Japan. Transactions and Proceedings of the Palaeontological Society of Japan, 184, 597-622.

Hirayama, R., Brinkman, D.B. \& Danilov, I.G. 2000. Distribution and biogeography of non-marine Cretaceous turtles. Russian Journal of Herpetology, 7, 181-198.

Hoffstetter, R. \& Gasc, J.-P. 1969. Vertebrae and ribs of modern reptiles. Pp. 201-310 in C. Gans, A. Bellairs and T.S. Parsons (eds) Biology of the Reptilia, Volume 1. Academic Press, London \& New York.

Hummel, K. 1929. Die fossilen Weichschildkröten (Trionychia). Geologische und Palaeontologische Abhandlungen, 16, 359-487.

Jamniczky, H.A., Brinkman, D.B. \& Russell, A.P. 2006. Phylogenetic implications of turtle cranial circulation: a review. Pp. 84-92 in I.G. Danilov and J.F. Parham (eds) Fossil Turtle Research, Vol. 1. Russian Journal of Herpetology, 13(Suppl.).

Joyce, W.G. 2003. A new Late Jurassic turtle specimen and the taxonomy of Palaeomedusa testa and Eurysternum wagleri. PaleoBios, 23(3), 1-8.

Joyce, W.G. 2004. Phylogeny, nomenclature, and ecology of Mesozoic turtles. Unpublished PhD 
Joyce, W.G. 2004. Phylogeny, nomenclature, and ecology of Mesozoic turtles. Unpublished PhD Dissertation, Yale University, New Haven, CT.

Joyce, W.G. 2007. Phylogenetic relationships of Mesozoic turtles. Bulletin of the Peabody Museum of Natural History, 48, 3-102.

Joyce, W.G. \& Gauthier, J.A. 2004. Palaeoecology of Triassic stem turtles sheds new light on turtle origins. Proceedings of the Royal Society of London, B, 271, 1-5.

Joyce, W.G., Parham, J.F. \& Gauthier, J.A. 2004. Developing a protocol for the conversion of rank-based taxon names to phylogenetically defined clade names, as exemplified by turtles. Journal of Paleontology, 78, 989-1013.

Joyce, W.G., Jenkins, F.A. Jr. \& Rowe, T. 2006. The presence of cleithra in the basal turtle Kayentachelys aprix. Pp. 93-103 in I.G. Danilov and J.F. Parham (eds) Fossil Turtle Research, Vol. 1. Russian Journal of Herpetology, 13(Suppl.).

Joyce, W.G., Lucas, S.G., Scheyer, T.M., Heckert, A.B. \& Hunt, A.P. 2009. A thin-shelled reptile from the Late Triassic of North America and the origin of the turtle shell. Proceedings of the Royal Society of London, B, 276, 507-513.

Kear, B.P. \& Lee, M.S.Y. 2006. A primitive protostegid from Australia and early sea turtle evolution. Biology Letters, 2, 116-119.

Khozatsky, L.I. 1997. Big turtle of the Late Cretaceous of Mongolia. Russian Journal of Herpetology, 4, 148-154.

Kitching, I.J., Forey, P.L., Humpries, C.J. \& Williams, D.M. 1998. Cladistics: the theory and practise of parsimony analysis - Second Edition. Oxford University Press, Oxford, $228 \mathrm{pp}$.

Klein, I.T. 1760. Klassification und kurze Geschichte der Vierfüßigen Thiere (translation by F. D. Behn). Jonas Schmidt, Lübeck.

Lambertz, M., Böhme, W. \& Perry, S.F. 2010. The anatomy of the respiratory system in Platysternon megacephalum Gray, 1831 (Testudines: Cryptodira) and related species, and its phylogenetic implications. Comparative Biochemistry and Physiology, Part A, 156, 330-336.

Lapparent de Broin, F. de. 2001. The European turtle fauna from the Triassic to the Present. Dumerilia, 4, 155-217.

Lapparent de Broin, F. de \& Murelaga, X. 1999. Turtles from the Upper Cretaceous of Laño (Iberian Peninsula). Estudios del Museo de Ciencias Naturales de Alava, 14(número especial 1), 135-211.
Lapparent de Broin, F. de, de la Fuente, M.S. \& Fernandez, M.S. 2007. Notoemys laticentralis (Chelonii, Pleurodira), Late Jurassic of Argentina: new examination of the anatomical structures and comparisons. Revue de Paléobiologie, 26, 99-136.

Laurin, M. \& Reisz, R.R. 1995. A reevaluation of early amniote phylogeny. Zoological Journal of the Linnean Society, 113, 165-223.

Lee, M.S.Y. 1993. The origin of the turtle body plan: bridging a famous morphological gap. Science, 261, 1716-1720.

Lee, M.S.Y. 1995. Historical burden in systematics and the interrelationships of 'parareptiles'. Biological Reviews of the Cambridge Philosophical Society, 70, 459-547.

Lee, M.S.Y. 1996. Correlated progression and the origin of turtles. Nature, 379, 812-815.

Lee, M.S.Y. 1997. Pareiasaur phylogeny and the origin of turtles. Zoological Journal of the Linnean Society, 120, 197-280.

Lee, M.S.Y. 2001. Molecules, morphology, and the monophyly of diapsid reptiles. Contributions to Zoology, 70, 1-22.

Li, C., Wu, X.-C., Rieppel, O., Wang, L.-T. \& Zhao, L.-J. 2008. An ancestral turtle from the Late Triassic of southwestern China. Nature, 456, 497-501.

Lipka, T.R., Therrien, F., Weishampel, D.B., Jamniczky, H.A., Joyce, W.G., Colbert, M.W. \& Brinkman, D.B. 2006. A new turtle from the Arundel Clay facies (Potomac Formation, Early Cretaceous) of Maryland, U.S.A. Journal of Vertebrate Paleontology, 26, 300-307.

Lydekker, R. 1889. Catalogue of the fossil reptilia and amphibia in the British Museum (Natural History) - Part III. The Order Chelonia. Trustees of the British Museum, London.

Lyson, T.R. \& Joyce, W.G. 2009. A new species of Palatobaena (Testudines: Baenidae) and a maximum parsimony and bayesian phylogenetic analysis of Baenidae. Journal of Paleontology, 83, 457-470.

Maddison, D.R. \& Maddison, W.P. 2001. MacClade. Version 4.01. Sinauer Associates, Sunderland, MA.

Matzke, A.T., Maisch, M.W., Sun, G.E., Pfretzschner, H. \& Stöhr, H. 2004. A new xijiangchelyid turtle (Testudines, Eucryptodira) from the Jurassic Qigu Formation of the Southern Junggar Basin, Xinjiang, North-West China. Palaeontology, 47, 1267-1299.

Meier, R. 1994. On the inappropriateness of the presence/absence recoding for non-additive multistate characters in computerized cladistic analyses. 
characters in computerized cladistic analyses. Zoologischer Anzeiger, 232, 201-212.

Meyer, H. von. 1860. Zur Fauna der Vorwelt. Reptilien aus dem lithographischen Schiefer des Jura in Deutschland und Frankreich. Heinrich Keller, Frankfurt am Main, 142 pp.

Meylan, P.A. 1988. Peltochelys Dollo and the relationships among the genera of the Carettochelyidae (Testudines: Reptilia). Herpetologica, 44, 440-450.

Meylan, P.A. \& Gaffney, E.S. 1989. The skeletal morphology of the Cretaceous cryptodiran turtle, Adocus, and the relationships of the Trionychoidea. American Museum Novitates, 2941, 1-60.

Meylan, P.A., Moody, R., Walker, C. \& Chapman, S. 2000. Sandownia harrisi, a highly derived Trionychoid turtle (Testudines: Cryptodira) from the Early Cretaceous of the Isle of Wight, England. Journal of Vertebrate Paleontology, 20, 522-532.

Milner, A.R. 2004. The turtles of the Purbeck Limestone Group of Dorset, Southern England. Palaeontology, 47, 1441-1467.

Müller, J. 2004. The relationships among diapsid reptiles and the influence of taxon selection. Pp. 379-408 in G. Arratia, M.V.H. Wilson and R. Cloutier (eds) Recent advances in the origin and early radiation of vertebrates. Verlag Dr. Friedrich Pfeil, Munich.

Müller, J. \& Reisz, R.R. 2005. An early captorhinid reptile (Amniota, Eureptilia) from the Upper Carboniferous of Hamilton, Kansas. Journal of Vertebrate Paleontology, 25, 561-568.

Müller, K. 2004. PRAP-computation of Bremer support for large data sets. Molecular Phylogenetics and Evolution, 31, 780-782.

Müller, K. 2007. PRAP, Parsimony Ratchet Analysis using PAUP*. Version 2.0b3 - <http://systevol.nees.unibonn.de/software/PRAP>.

Nessov, L.A. 1995. On some Mesozoic turtles of the Fergana Depression (Kyrgyzstan) and Dzhungar Alatau Ridge (Kazakhstan). Russian Journal of Herpetology, 2, 134-141.

Nixon, K.C. 1999. The Parsimony Ratchet, a new method for rapid parsimony analysis. Cladistics, 15, 407-414.

Parham, J.F. \& Hutchison, J.H. 2003. A new eucryptodiran turtle from the Late Cretaceous of North America (Dinosaur Provincial Park, Alberta, Canada). Journal of Vertebrate Paleontology, 23, 783-798.
Parham, J.F., Feldman, C.R. \& Boore, J.L. 2006. The complete mitochondrial genome of the enigmatic bigheaded turtle (Platysternon): description of unusual genomic features and the reconciliation of phylogenetic hypotheses based on mitochondrial and nuclear DNA. BMC Evolutionary Biology, 6, 11.

Peng, J.-H. \& Brinkman, D.B. 1993. New material of Xinjiangchelys (Reptilia: Testudines) from the Late Jurassic Qigu Formation (Shishugou Group) of the Pingfengshan locality, Junggar Basin, Xinjiang. Canadian Journal of Earth Sciences, 30, 2013-2026.

Pimentel, R.A. \& Riggins, R. 1987. The nature of cladistic data. Cladistics, 3, 201-209.

Pleijel, F. 1995. On character coding for phylogeny reconstruction. Cladistics, 11, 309-315.

Reisz, R.R. \& Head, J.J. 2008. Turtle origins out to sea. Nature, 456, 450-451.

Reisz, R.R. \& Laurin, M. 1991. Owenetta and the origin of turtle. Nature, 349, 324-326.

Reisz, R.R. \& Scott, D. 2002. Owenetta kitchingorum, sp. nov., a small parareptile (Procolophonia: Owenettidae) from the Lower Triassic of South Africa. Journal of Vertebrate Paleontology, 22, 244-256.

Reisz, R.R., Berman, D.S. \& Scott, D. 1984. The anatomy and relationships of the Lower Permian reptile Araeoscelis. Journal of Vertebrate Paleontology, 4, 57-67.

Rieppel, O. 1980. The skull of the Upper Jurassic cryptodire turtle Thalassemys, with a reconsideration of the chelonian braincase. Palaeontographica, Abteilung A, 171, 105-140.

Rieppel, O. 1993. Studies on skeleton formation in reptiles: patterns of ossification in the skeleton of Chelydra serpentina (Reptilia, Testudines). Journal of Zoology, London, 231, 487-509.

Rieppel, O. 1994. Osteology of Simosaurus gaillardoti and the relationships of stem-group Sauropterygia. Fieldiana - Geology, 28, 1-85.

Rieppel, O. 2008. The relationships of turtles within amniotes. Pp. 345-353 in J. Wyneken, M.H. Godfrey and V. Bels (eds) Biology of turtles. CRC Press, Boca Raton, FL.

Rieppel, O. \& deBraga, M. 1996. Turtles as diapsid reptiles. Nature, 384, 453-455.

Rieppel, O. \& Reisz, R.R. 1999. The origin and early evolution of turtles. Annual Review of Ecology and Systematics, 30, 1-22.

Romer, A.S. 1956. Osteology of the Reptiles. University of Chicago Press, Chicago, 772 pp. 
Rougier, G.W., de la Fuente, M.S. \& Arcucci, A.B. 1995. Late Triassic turtles from South America. Science, 268, 855-858.

Scheyer, T.M. \& Anquetin, J. 2008. Bone histology of the Middle Jurassic turtle shell remains from Kirtlington, Oxfordshire, England. Lethaia, 41, 85-96.

Shaffer, H.B., Meylan, P.A. \& McKnight, M.L. 1997. Tests of turtle phylogeny: molecular, morphological, and paleontological approaches. Systematic Biology, 46, 235-268.

Sharkey, M.J. \& Leathers, J.W. 2001. Majority does not rule: the trouble with majority-rule consensus trees. Cladistics, 17, 282-284.

Siebenrock, F. 1897. Das Kopfskelett der Schildkröten. Sitzungsberichte der Königliche Akademie der Wissenschaften, Mathematisch-naturwissenschaftliche Klasse, Wien, 106, 245-328.

Sikes, D.S. \& Lewis, P.O. 2001. PAUPRat: PAUP* implementation of the parsimony ratchet. Beta software, version 1. Distributed by the authors. Department of Ecology and Evolutionary Biology, University of Connecticut, Storrs, USA.

Sterli, J. 2008. A new, nearly complete stem turtle from the Jurassic of South America with implications for turtle evolution. Biology Letters, 4, 286-289.

Sterli, J. \& de la Fuente, M.S. 2010. Anatomy of Condorchelys antiqua Sterli, 2008, and the origin of the modern jaw closure mechanism in turtles. Journal of Vertebrate Paleontology, 30, 351-366.

Sterli, J. \& Joyce, W.G. 2007. The cranial anatomy of the Early Jurassic turtle Kayentachelys aprix. Acta Palaeontologica Polonica, 52, 675-694.

Sterli, J., de la Fuente, M.S. \& Rougier, G.W. 2007. Anatomy and relationships of Palaeochersis talampayensis, a Late Triassic turtle from Argentina. Palaeontographica, Abteilung A, 281, 1-61.

Sterli, J., Müller, J., Anquetin, J. \& Hilger, A. In press. The parabasisphenoid complex in Mesozoic turtles and the evolution of the testudinate basicranium. Canadian Journal of Earth Sciences.

Sukhanov, V.B. 2000. Mesozoic turtles of Middle and Central Asia. Pp. 309-367 in M.J. Benton, M.A. Shishkin, D.M. Unwin and E.N. Kurochkin (eds) The age of dinosaurs in Russia and Mongolia. Cambridge University Press, Cambridge.

Sukhanov, V.B. 2006. An archaic turtle, Heckerochelys romani gen. et sp. nov., from the Middle Jurassic of Moscow region, Russia. Pp. 112-118 in I.G. Danilov and J.F.
Parham (eds) Fossil Turtle Research, Vol. 1. Russian Journal of Herpetology, 13(Suppl.).

Sukhanov, V.B. \& Narmandakh, P. 1974. New Early Cretaceous turtle from the continental deposits of the Northern Gobi. The Joint Soviet-Mongolian Paleontological Expedition Transactions, 1, 192-220.

Sukhanov, V.B. \& Narmandakh, P. 2006. New taxa of Mesozoic turtles from Mongolia. Pp. 119-127 in I.G. Danilov and J.F. Parham (eds) Fossil Turtle Research, Vol. 1. Russian Journal of Herpetology, 13(Suppl.).

Sumrall, C.D., Brochu, C.A. \& Merck, J.W. Jr. 2001. Global lability, regional resolution, and majority-rule consensus bias. Paleobiology, 27, 254-261.

Swofford, D.L. 2002. PAUP. Version 4.0b10. Sinauer Associates, Sunderland, MA.

Thorley, J.L. \& Page, R.D.M. 2000. RadCon: phylogenetic tree comparison and consensus. Bioinformatics, 16, 486-487.

Tong, H., Buffetaut, E. \& Suteethorn, V. 2002. Middle Jurassic turtles from southern Thailand. Geological Magazine, 139, 687-697.

Wagner, A. 1861. Neue Beiträge zur Kenntniss der urweltlichen Fauna des lithographischen Schiefers. Abhandlungen der mathemat.-physikalischen Classe der königlich bayerischen Akademie der Wissenschaften, 9, 67-124.

Walther, W.G. 1922. Die Neu-Guinea-Schildkröte Carettochelys insculpta Ramsay. Nova Guinea (Zoology), 13, 607-704.

Wilkinson, M. 1994. Common cladistic information and its consensus representation: reduced Adams and reduced cladistic consensus trees and profiles. Systematic Biology, 43, 343-368.

Wilkinson, M. 1995a. More on reduced consensus methods. Systematic Biology, 44, 435-439.

Wilkinson, M. 1995b. Coping with missing entries in phylogenetic inference using parsimony. Systematic Biology, 44, 501-514.

Wilkinson, M. 1995c. A comparison of two methods of character construction. Cladistics, 11, 297-308.

Wilkinson, M. 2001. TAXEQ3: software and documentation. Department of Zoology, The Natural History Museum, London.

Wilkinson, M. 2003. Missing entries and multiple trees: instability, relationships, and support in parsimony analysis. Journal of Vertebrate Paleontology, 23, 311-323.

Wings, O. \& Joyce, W.G. 2009. An exceptionally large Jurassic taphocoenosis from Xinjiang Autonomous 
Province, China. Journal of Vertebrate Paleontology, 29(3), 202A.

Woodward, A.S. 1901. On some extinct reptiles from Patagonia, of the genera Miolania, Dinilysia, and Genyodestes. Proceedings of the Zoological Society of London, 1901, 169-184.

Ye, X. 1982. Middle Jurassic turtles from Sichuan, SW China. Vertebrata Palasiatica, 20, 282-290.

Ye, X. 1986. A Jurassic turtle from Junggar, Xinjiang. Vertebrata Palasiatica, 24, 171-181.

Ye, X. 1990. Chengyuchelyidae, n. fam., Middle Jurassic turtles of China. Studia Geologica Salmanticensia, Volumen Especial (Studia Palaeocheloniologica), 3, 33-40.

Ye, X. 1994. Fossil and recent turtles of China. Science Press, Beijing, $112 \mathrm{pp}$.

Ye, Y. \& Pi, X. 1997. A new genus of Chengyuchelyidae from Dashanpu, Zigong, Sichuan. Vertebrata Palasiatica, 35, 182-188.

Young, C.C. \& Chow, M.C. 1953. New fossil reptiles from Szechuan, China. Acta Scientia Sinica, 2, 216-229.

Zangerl, R. 1939. The homology of the shell elements in turtles. Journal of Morphology, 65, 383-409.

Zangerl, R. 1969. The turtle shell. Pp. 311-339 in C. Gans, A. Bellairs and T.S. Parsons (eds) Biology of the Reptilia, Volume 1. Academic Press, London \& New York. 